University of Nebraska - Lincoln

DigitalCommons@University of Nebraska - Lincoln

Timothy J. Gay Publications

Research Papers in Physics and Astronomy

4-1-1992

\title{
Electron production in proton collisions with atoms and molecules: energy distributions
}

\author{
M. Eugene Rudd \\ University of Nebraska-Lincoln, erudd@unl.edu \\ Y.-K. Kim \\ National Institute of Standards and Technology, Gaithersburg, Maryland \\ D. H. Madison \\ University of Missouri-Rolla, Rolla, Missouri \\ Timothy J. Gay \\ University of Nebraska - Lincoln, tgay1@unl.edu
}

Follow this and additional works at: https://digitalcommons.unl.edu/physicsgay

Part of the Physics Commons

Rudd, M. Eugene; Kim, Y.-K.; Madison, D. H.; and Gay, Timothy J., "Electron production in proton collisions with atoms and molecules: energy distributions" (1992). Timothy J. Gay Publications. 36.

https://digitalcommons.unl.edu/physicsgay/36

This Article is brought to you for free and open access by the Research Papers in Physics and Astronomy at DigitalCommons@University of Nebraska - Lincoln. It has been accepted for inclusion in Timothy J. Gay Publications by an authorized administrator of DigitalCommons@University of Nebraska - Lincoln. 
Rev. Mod. Phys. 64, 441 - 490 (1992)

[Issue 2 - April 1992]

\title{
Electron production in proton collisions with atoms and molecules: energy distributions
}

\author{
M. E. Rudd \\ Department of Physics and Astronomy, Behlen Laboratory, University of \\ Nebraska, Lincoln, Nebraska 68588 \\ Y. -K. Kim \\ National Institute of Standards and Technology, Gaithersburg, Maryland 20899 \\ D. H. Madison and T. J. Gay \\ Physics Department and Laboratory for Atomic and Molecular Research, \\ University of Missouri-Rolla, Rolla, Missouri 65401
}

\begin{abstract}
All known data on the energy distribution of secondary electrons from collisions of protons with atoms and molecules have been reviewed and differential cross sections have been collected. The two experimental methods used to obtain the data are discussed and possible sources of error pointed out. Theoretical treatments are reviewed and several methods of checking the consistency of the data are discussed. Two semiempirical models have been chosen to represent the differential cross sections, and parameters for these models are given which fit the average of the experimental data, subject to known constraints. Recommended values of differential cross sections are given for ten target gases by means of these models.
\end{abstract}

(C)1992 The American Physical Society

URL: http://link.aps.org/abstract/RMP/v64/p441

DOI: $10.1103 /$ RevModPhys.64.441 


\title{
Electron production in proton collisions with atoms and molecules: energy distributions
}

\author{
M. E. Rudd
}

Department of Physics and Astronomy, Behlen Laboratory, University of Nebraska, Lincoln, Nebraska 68588

Y.-K. Kim

National Institute of Standards and Technology, Gaithersburg, Maryland 20899

D. H. Madison and T. J. Gay

Physics Department and Laboratory for Atomic and Molecular Research, University of Missouri-Rolla, Rolla, Missouri 65401

\begin{abstract}
All known data on the energy distribution of secondary electrons from collisions of protons with atoms and molecules have been reviewed and differential cross sections have been collected. The two experimental methods used to obtain the data are discussed and possible sources of error pointed out. Theoretical treatments are reviewed and several methods of checking the consistency of the data are discussed. Two semiempirical models have been chosen to represent the differential cross sections, and parameters for these models are given which fit the average of the experimental data, subject to known constraints Recommended values of differential cross sections are given for ten target gases by means of these models.
\end{abstract}

\section{CONTENTS}

I. Introduction

442

II. Definitions

III. Qualitative Considerations

A. Sign of charge

B. Projectile momentum

C. Energy transferred to an ejected electron

D. Slow incident particles

E. Rutherford cross section

F. Binary collision peak

G. Soft and hard collisions

H. Optical (zero-momentum-transfer) limit

I. Semiclassical treatment and the plane-wave Born approximation

IV. Theoretical Treatments

A. Binary-encounter approximation

B. Classical-trajectory Monte Carlo method

C. Quantum-mechanical methods

1. Weak initial- and final-state electron-projectile interactions

2. Strong initial-state interactions and weak finalstate interactions

3. Strong electron-projectile interactions

a. Continuum-distorted-wave method

b. Other methods for strong electron-projectile interactions

D. Comparison of experiment and theory

V. Consistency Checks

A. Bethe theory

B. Platzman plots

C. Comparison with electron-impact data

D. Comparison with photoionization data

E. Comparison with total ionization cross sections

F. Relationship with stopping cross sections

VI. Semiempirical Models
A. Miller's model
B. Dillon-Inokuti model
C. Kim's model
D. Rudd's mode

VII. Experimental Methods

A. Analysis of ejected electrons
B. Ion energy-loss spectroscopy

VIII. Error Analysis

A. Analysis of ejected electrons

1. Total signal and background counts, $N_{S}$ and $N_{B}$

2. Number density of target gas, $n$

3. The "absorption" coefficient $\sigma_{s} x$

4. Number of protons $N_{0}$

5. Integrated path length/solid angle $(n l \Omega)_{\text {eff }}$

6. Energy width-transmission product of the analyzer, $\Delta W t$

7. Detection efficiency $\epsilon$

B. Ion energy-loss spectroscopy

1. The beam-related signal-background difference $N_{S}-N_{B}$

2. The target length-density product $n l$

3. The number of incident protons $N_{0}$

IX. Recommended Values of Singly-Differential Cross Sections

A. Choice of model $\quad 478$

B. Data adjustment 478

C. Fitting of the Rudd model 479

D. Table of parameters in the Rudd model 479

E. Recommended values

X. Discussion of Singly-Differential Cross-Section Data for Individual Targets

A. Helium

B. Neon

C. Argon

D. Krypton

E. Molecular hydrogen

F. Molecular nitrogen

G. Molecular oxygen

H. Carbon dioxide

I. Water vapor

J. Methane

K. Other targets

XI. Recommendations for future work

Acknowledgments

Appendix A: Maximum Energy Transferred to an Electron at Rest

Appendix B: Binary Peak Angle

References 


\section{INTRODUCTION}

When an energetic ion such as a proton collides with an atom or a molecule, several inelastic processes can take place in addition to elastic scattering. The basic ones are excitation of the target, electron capture, and the ejection of free electrons from the target. The last process, often called "direct ionization," involves the greatest exchange of energy and is also the most probable process for collision velocities exceeding the orbital velocity of the electron in the target. This process is the subject of the present review.

Because of the relatively large energy transfer, ionization is a very important process for any study involving the energy loss of particles traversing matter and for investigations of the deposition of energy in matter. Examples are the radiation damage of biological and other materials, radiation detection devices, and investigations of the aurora borealis and other upper atmospheric phenomena. Studies of magnetic and inertial-confinement fusion and of stellar atmospheres also make use of ionization data.

Knowledge of the total ionization cross section as a function of incident energy suffices for some purposes. In an earlier paper Rudd, Kim, Madison, and Gallagher (1985) made a critical review of total cross-section measurements for proton-impact ionization. In that review recommendations were made for values of cross sections over a wide energy range for 13 target gases, the theoretical and semiempirical methods available for calculation of these cross sections were discussed, and a semiempirical equation was given along with parameters to enable the cross sections to be easily computed for a given energy.

The present paper extends that review to differential cross sections, which are crucial for a wide variety of problems. In the calculation of radiation damage, for example, it is necessary to take into account the ionization caused by the secondary electrons themselves. For such purposes, the energy spectrum of secondary electrons is needed. This information is contained in the singlydifferential cross sections (SDCS's), i.e., cross sections as functions of the secondary-electron energy. For other purposes, such as studies of track structure and radial dose distributions, the angular distribution of electrons with a given energy is also needed. This requires doubly-differential cross sections (DDCS's), i.e., cross sections differential in both the angle and the energy of the ejected secondary electrons. There is also another SDCS, namely the cross section as a function of angle only, which may be obtained by integrating DDCS data over electron energy. However, this cross section has found fewer applications in the above-mentioned areas.

Unfortunately, there is no reliable and direct way to measure energy distributions of secondary electrons. The most popular method is to measure the DDCS over a wide range of angles and then to integrate it over the electron ejection angle. However, this method is often marred by uncertainties in the forward and backward directions and in the spectra of slow ejected electrons. The latter uncertainty is more serious because slow electrons significantly contribute to the total ionization cross section.

Differential cross sections for electron ejection are not only of interest because of their many applications, but are also important in their own right, since they provide more detailed information about basic ionization processes than do total cross sections.

The basic mechanisms of electron ejection are known, and theoretical treatments exist that will yield the required cross sections over some ranges of parameters. However, no universal method of calculation exists that yields accurate cross sections for all primary and secondary energies and for all targets. This makes it necessary to rely on experimental data and on semiempirical models. Even though there is general agreement among most of the existing experimental data sets, there are significant discrepancies. As a result, a potential user is often faced with the problem of choosing from conflicting sets of data often covering different ranges of parameters. In the present review the experimental data are critically evaluated in the light of well-established theoretical results and of their consistency with other related data. Recommended values for the SDCS's for ten common target gases are presented.

In Sec. II basic cross sections are defined and in Sec. III qualitative features of the differential cross sections are discussed. $A b$ initio theoretical treatments and their limitations are discussed in Sec. IV, and the various methods for making consistency checks on experimental data are considered in Sec. V. Semiempirical models for SDCS's are discussed in Sec. VI. In Secs. VII and VIII experimental methods are presented and sources of error analyzed. The recommended values of the SDCS's are given in Sec. IX. Section $X$ contains a discussion of the SDCS data for individual targets and gives the results in graphical form. Recommendations for future work are offered in Sec. XI.

\section{DEFINITIONS}

The collision of a fast charged particle with a neutral atom or molecule may result in the ejection of one or more secondary electrons, which, in the case of proton impact, must come from the target. These electrons are ejected over a range of energies and directions.

The differential cross section is usually measured by directing a beam, in this case of protons, through a gas target with low number density $n$, and measuring the electrons having an energy $W$ that are ejected in a given direction from a known length of path $l$. If one measures the number $N$ of electrons ejected into a solid angle $\Delta \Omega$ with energies $W$ to $W+\Delta W$, then the doubly-differential cross section is defined by the equation

$$
d^{2} \sigma / d W d \Omega=N /\left(N_{0} n l \Delta \Omega \Delta W\right),
$$


where $N_{0}$ is the number of incident particles. For unpolarized beams and targets, the cross section is independent of the azimuthal angle $\phi$ and depends only on the polar angle $\theta$ measured relative to the forward direction of the incident beam. In this case, the DDCS is a function only of $\theta$ and $W$.

If the DDCS is measured over a sufficiently wide range of angles and energies, then one may obtain by numerical integration the SDCS representing the energy distribution,

$$
\frac{d \sigma}{d W}=\int \frac{d^{2} \sigma}{d W d \Omega} d \Omega
$$

or the SDCS giving the angular distribution,

$$
\frac{d \sigma}{d \Omega}=\int_{0}^{W_{\max }} \frac{d^{2} \sigma}{d W d \Omega} d W
$$

where $W_{\max }=T_{0}-I$ is the maximum kinetic energy of the ejected electrons, $T_{0}$ is the energy of the incident proton, and $I$ is the ionization potential. The total cross section for electron ejection is obtained by integrating the DDCS twice:

$$
\sigma_{-}=\int_{0}^{W_{\max }} \int \frac{d^{2} \sigma}{d W d \Omega} d \Omega d W .
$$

While other methods have been used to measure the total cross section (Rudd et al., 1985), no direct measurements have been reported of singly-differential cross sections for proton impact except for those of Park and co-workers (Park and Schowengerdt, 1969b; Park et al., 1977). Vroom et al. (1977) have devised a method for direct SDCS measurements with electron impact. This technique has not been pursued, however, and few data thus obtained exist.

Knowledge of the differential cross sections, especially $d \sigma / d W$, makes possible the calculation of several additional quantities that are of interest in studies of radiation effects and in other areas where the deposition of energy by fast charged particles needs to be known. The average energy of secondary electrons is

$$
W_{\mathrm{av}}=\left(1 / \sigma_{-}\right) \int_{0}^{W_{\max }} W \frac{d \sigma}{d W} d W .
$$

The stopping cross section due to ionization is

$$
\sigma_{\mathrm{st}}=(1 / R) \int_{0}^{W_{\max }}(W+I) \frac{d \sigma}{d W} d W,
$$

where $R$ is the rydberg energy $(13.6 \mathrm{eV})$. If there is more than one shell in the target, this equation must be replaced by the summation

$$
\sigma_{\mathrm{st}}=(1 / R) \sum_{i} \int_{0}^{W_{\max , i}}\left(W+I_{i}\right) \frac{d \sigma_{i}}{d W} d W,
$$

where $I_{i}$ is the binding energy of the $i$ th shell, $W_{\max , i}$ is the maximum kinetic energy of electrons ejected from the $i$ th shell, and $d \sigma_{i} / d W$ is the partial cross section for ejecting an electron from the $i$ th shell. The fraction $f_{W}$ of electrons ejected with an energy greater than a given energy $W$ is

$$
f_{W}=\left(1 / \sigma_{-}\right) \int_{W}^{W_{\max }} \frac{d \sigma}{d W^{\prime}} d W^{\prime} .
$$

This quantity is especially useful in finding the fraction of electrons with $W \geq I$, since such electrons can cause further ionization.

\section{QUALITATIVE CONSIDERATIONS}

In this section, we consider qualitatively the basic physics of proton-impact ionization. We also consider electron-impact ionization, because comparisons between electron and proton data have proven very useful in evaluating and understanding proton measurements.

\section{A. Sign of charge}

The plane-wave Born approximation (PWBA), in which the incident particle is represented by a plane wave, predicts that both excitation and ionization cross sections will be proportional to $Z^{2}$, where $Z$ is the projectile charge. As a result, the PWBA predicts the same cross section for a charged particle of a given mass and velocity regardless of its sign of charge. In the highenergy region where the PWBA is valid and does not depend on projectile mass, only the velocity of the particle is relevant. For example, antiprotons and positrons with the same high speed produce the same target ionization. At intermediate to low energies, however, mass and charge-sign differences, e.g., between protons and electrons, cause cross-section differences. Experimental data with fast projectiles are often scrutinized for these expected trends as signs of their consistency and reliability.

\section{B. Projectile momentum}

Since a proton is 1836 times more massive than an electron, an incident proton carries that much more momentum than an electron of the same speed. The description of a continuum wave function depends on the momentum $k$, rather than the speed $v$, of the projectile, and the proton wave function approaches a plane wave of the same momentum in the limit of high $k$. As a result, the PWBA, which uses plane waves for the projectile, works better for proton-impact cross sections than it does for electrons of the same speed, for projectile speeds below the high-energy region discussed in Sec. III.A.

\section{Energy transferred to an ejected electron}

An incident proton can transfer most of its energy to a bound electron, but in practice the energy transfer is usually far less than the incident energy. The case of electron-impact ionization is different, however, since one cannot distinguish the two or more electrons emerging after an ionizing collision. As a result, an operational dis- 
tinction is made between the primary (fast) electron and secondary (slow) electron by requiring the maximum energy transferred $W_{\max }$ to be one-half of the incident energy $T_{0}$ after subtracting the required binding energy $I$, i.e.,

$$
W_{\max }\left(e^{-}\right)=\left(T_{0}-I\right) / 2 .
$$

This choice of $W_{\max }\left(e^{-}\right)$assumes that the electron exchange effect between the incident and ejected electrons has been properly treated.

For incident protons, conservation of energy and momentum restricts the maximum energy transfer to an unbound electron at rest to (see Appendix A)

$$
W_{\max }(p)=2 m v_{0}^{2}=4 T,
$$

where $m$ is the electron mass, $v_{0}$ is the incident-proton speed, and $T=m v_{0}^{2} / 2$. This limit is independent of the projectile mass $M$ (assuming $M \gg m$ ) and is known as the free-electron limit for energy transfer by heavy projectiles. In reality, a bound electron can exceed this limit because the ion core can recoil, imparting additional momentum and hence higher kinetic energy to the ejected electron. We emphasize here that the recoil of the ion core involves large momentum because of the ion mass, but that the recoil ion does not receive an appreciable amount of kinetic energy from the incident proton. As will be demonstrated later in numerous examples, the production of very energetic electrons by fast incident protons drops sharply beyond this free-electron limit. From Eq. (B7) of Appendix B we see that the freeelectron energy limit for electrons ejected in directions other than that of the incident beam will be less than the maximum given by Eq. (10), which is, in turn, far less than the incident energy of the proton, $T_{0}$.

\section{Slow incident particles}

When projectile speeds are sufficiently low ( $v \lesssim 3$ a.u.), the "two-center" (ionized target plus receding projectile) nature of the collision complex has an important effect on the dynamics of electron ejection. While negative projectiles such as electrons repel ionized target electrons, protons attract them, causing an enhancement in the doubly-differential cross sections at forward-ejection angles. This attraction results in two mechanisms, or "channels", for ionization unavailable to negativeprojectile collision complexes: charge transfer to the continuum (more recently labeled electron capture to the continuum - see, for example, Crooks and Rudd, 1970; Macek, 1970), in which the ejected electron has a velocity closely matching that of the projectile, and "saddlepoint" ionization (Olson et al., 1987), where electrons stranded on or near the saddle point of the electric potential between the positive target ion and receding projectile emerge with roughly half the projectile velocity. While ionization due to charge transfer to the continuum contributes little to the total cross section, the saddle- point mechanism provides a significant fraction of the ionized electrons and is responsible for differences in the cross sections between, say, protons and antiprotons (see Kimura and Inokuti, 1988; Olson and Gay, 1988; Fainstein et al., 1989b; Schultz, 1989).

\section{E. Rutherford cross section}

The collision of a charged particle with another at rest is described by the Rutherford scattering formula, which can be written

$$
\frac{d \sigma}{d W}=\left(4 \pi a_{0}^{2} / T\right)(R / W)^{2},
$$

where $W$ is the kinetic energy of the target particle after the collision, $T$ is the kinetic energy of an electron with the same speed as the incident particle $\left[T=(m / M) T_{0}\right.$ and hence $T=T_{0}$ only for incident electrons or positrons; see Appendix A], and $a_{0}$ is the Bohr radius (0.529 $\AA$ ). This formula is obtained from the usual expression for the Rutherford scattering cross section in terms of $\theta$ (Landau and Lifshitz, 1965) by assuming all momentum transferred to the target electron remains with it upon ejection from the atom, as would be the case for a free electron. This is the correct quantum-mechanical result for electrons at rest, which is singular at $W=0$. A bound atomic electron, however, is not at rest, and part of the energy transfer must be used to overcome the binding, with the remainder going into kinetic energy. Consequently it is logical to replace $W$ by the energy transfer $E$ needed to eject an electron of energy $W$,i.e.,

$$
E=W+I \text {. }
$$

With this substitution, the singularity in the original Rutherford cross section is removed. The modified Rutherford cross section, which is an approximation to the original but singular Rutherford scattering cross section, is given by

$$
\frac{d \sigma_{R}}{d W}=\left(4 \pi a_{0}^{2} / T\right)(R / E)^{2} .
$$

We shall refer to the "modified" Rutherford cross section, Eq. (13), as the Rutherford cross section hereafter for brevity, while Eq. (11) will be referred to as the "original" Rutherford cross section. The modified Rutherford cross section will, of course, approach the original Rutherford cross section when $W \gg I$. For a target atom or molecule with complex shell structure, Eq. (12) is not unique because most experiments measure only $W$ without distinguishing the shell from which an ejected electron comes. As is shown later, Eq. (12) must be expanded and $E$ must be defined for each subshell in such a case. The expanded form will be used, however, only to help in the understanding of the qualitative behavior of singly-differential cross sections.

\section{F. Binary collision peak}

In doubly-differential cross-section measurements, the energy and angular distributions of the ejected electrons 
TABLE I (a). Binding $(I)$ and kinetic $(U)$ energies $(\mathrm{eV})$ and occupation number $(N)$ of some rare gases and diatomic molecules. Binding energies are experimental data, and the kinetic energies are those calculated from nonrelativistic Hartree-Fock wave functions.

\begin{tabular}{|c|c|c|c|c|c|c|c|c|c|c|c|c|c|c|c|}
\hline \multirow[b]{2}{*}{ Shell } & \multicolumn{3}{|c|}{$\mathrm{He}$} & \multicolumn{3}{|c|}{$\mathrm{Ne}$} & \multicolumn{3}{|c|}{$\mathrm{Ar}$} & \multicolumn{3}{|c|}{$\mathrm{Kr}$} & \multicolumn{3}{|c|}{$\mathrm{Xe}$} \\
\hline & $I$ & $U$ & $N$ & $I$ & $U$ & $N$ & $I$ & $U$ & $N$ & $I$ & $U$ & $N$ & $I$ & $U$ & $N$ \\
\hline $1 s$ & 24.59 & 39.51 & 2 & 866.9 & 1259.1 & 2 & 3202.9 & 4192.9 & 2 & 14325.6 & 17146.1 & 2 & 3456.4 & 38899.6 & 2 \\
\hline $2 s$ & & & & 48.47 & 141.88 & 2 & 326.0 & 683.1 & 2 & 1921.0 & 3406.9 & 2 & 5452.8 & 9240.0 & 2 \\
\hline $2 p$ & & & & 21.60 & 116.02 & 6 & 249.18 & 651.4 & 6 & 1692.3 & 3375.0 & 6 & 4889.4 & 8229.7 & 6 \\
\hline $3 s$ & & & & & & & 29.24 & 103.5 & 2 & 295.2 & 829.8 & 2 & 1093.2 & 2481.8 & 2 \\
\hline $3 p$ & & & & & & & 15.82 & 78.07 & 6 & 216.8 & 773.7 & 6 & 957.7 & 2412.9 & 6 \\
\hline $3 d$ & & & & & & & & & & 93.0 & 650.3 & 10 & 672.3 & 2283.8 & 10 \\
\hline $4 s$ & & & & & & & & & & 27.5 & 115.8 & 2 & 213.8 & 696.9 & 2 \\
\hline $4 p$ & & & & & & & & & & 14.22 & 82.72 & 6 & 146.7 & 635.3 & 6 \\
\hline $4 d$ & & & & & & & & & & & & & 68.21 & 495.7 & 10 \\
\hline $5 s$ & & & & & & & & & & & & & 23.3 & 110.4 & 2 \\
\hline $5 p$ & & & & & & & & & & & & & 12.56 & 79.74 & 6 \\
\hline & & $\mathrm{H}_{2}$ & & & $\mathrm{~N}_{2}$ & & & $\mathrm{O}_{2}$ & & & & & & & \\
\hline & $I$ & $U$ & $N$ & $I$ & $U$ & $N$ & $I$ & $U$ & $N$ & & & & & & \\
\hline $1 \sigma_{g}$ & 15.43 & 31.96 & 2 & 409.9 & 601.78 & 2 & 543.5 & 794.84 & 2 & & & & & & \\
\hline $1 \sigma_{u}^{\circ}$ & & & & 409.9 & 602.68 & 2 & 543.5 & 795.06 & 2 & & & & & & \\
\hline $2 \sigma_{g}$ & & & & 37.3 & 69.53 & 2 & 40.3 & 78.19 & 2 & & & & & & \\
\hline $2 \sigma_{u}$ & & & & 18.78 & 62.45 & 2 & 25.69 & 90.40 & 2 & & & & & & \\
\hline $1 \pi_{u}$ & & & & 16.96 & 55.21 & 4 & 18.88 & 72.24 & 4 & & & & & & \\
\hline $3 \sigma_{g}$ & & & & 15.59 & 44.27 & 2 & 16.42 & 60.08 & 2 & & & & & & \\
\hline $1 \pi_{g}$ & & & & & & & 12.07 & 82.14 & 2 & & & & & & \\
\hline
\end{tabular}


TABLE I(b). Binding $(I)$ and kinetic $(U)$ energies $(\mathrm{eV})$ and occupation number $(N)$ of some polyatomic molecules. The binding energies are mostly experimental values, and the kinetic energies are calculated values using DZP (double $\xi+$ polarization) basis sets. [For the DZP basis set, see Stevens et al. (1984) for $\mathrm{SF}_{6}$ and $\mathrm{TeF}_{6}$, and Dunning and Hay (1977), pp. 1-27.] MO: molecular orbital. Experimental values are marked by an asterisk. [Most experimental binding energies (vertical ionization potentials) are from Berkowitz (1979) except for $\mathbf{N H}_{2}$ (Gibson et al., 1985), $\mathbf{C H}_{3}$ (Dyke et al., 1976), $\mathbf{C O}_{2}$ (Samson and Gardner, 1973), SF $\left(\mathbf{P o t t s}^{\text {et al., }}\right.$ 1970), and $\left(\mathrm{CH}_{3}\right)_{2} \mathrm{NH}$ (Vovna and Vilesov, 1974).]

\begin{tabular}{|c|c|c|c|c|c|c|c|}
\hline MO & $I$ & $U$ & $N$ & MO & $I$ & $U$ & $N$ \\
\hline \multicolumn{4}{|c|}{$\mathbf{N H}_{2}$} & \multicolumn{4}{|c|}{$\mathrm{C}_{6} \mathrm{H}_{6}$} \\
\hline $1 a_{1}\left(\begin{array}{ll}N & 1 s)\end{array}\right.$ & 423.8 & 1204.6 & 2 & $1 a_{1 g}(C 1 s)$ & $290.2^{*}$ & 871.1 & 2 \\
\hline $2 a_{1}$ & 30.09 & 100.9 & 2 & $1 e_{1 u}(C 1 s)$ & $290.2^{*}$ & 871.4 & 4 \\
\hline $1 b_{1}$ & 17.47 & 72.25 & 2 & $1 e_{2 g}(C 1 s)$ & $290.2^{*}$ & 872.2 & 4 \\
\hline $3 a_{1}$ & 13.02 & 87.96 & 2 & $1 b_{1 u}(C 1 s)$ & $290.2^{*}$ & 872.6 & 2 \\
\hline \multirow[t]{3}{*}{$1 b_{2}$} & $11.14^{*}$ & 46.86 & 1 & $2 a_{1 g}$ & $26.0^{*}$ & 78.86 & 2 \\
\hline & & & & $1 e_{1 u}$ & $22.7^{*}$ & 85.31 & 4 \\
\hline & \multicolumn{2}{|c|}{$\mathrm{CH}_{3}$} & & $2 e_{2 g}$ & $19.0^{*}$ & 77.99 & 4 \\
\hline $1 a_{1}\left(\begin{array}{ll}C & 1 s)\end{array}\right)$ & 305.5 & 872.0 & 2 & $3 a_{1 g}$ & $16.9^{*}$ & 50.21 & 2 \\
\hline $2 a_{1}$ & 24.57 & 68.84 & 2 & $2 b_{1 u}$ & $15.4^{*}$ & 69.15 & 2 \\
\hline $1 e$ & $15.10^{*}$ & 52.74 & 4 & $1 b_{2 u}$ & $14.8^{*}$ & 76.97 & 2 \\
\hline \multirow[t]{3}{*}{$3 a_{1}$} & $9.84^{*}$ & 29.73 & 1 & $3 e_{1 u}$ & $14.0^{*}$ & 64.50 & 4 \\
\hline & & & & $1 a_{2 u}$ & $12.3^{*}$ & 47.90 & 2 \\
\hline & & & & $3 e_{2 g}$ & $11.8^{*}$ & 74.98 & 4 \\
\hline $1 \sigma_{g}(C 1 s)$ & $291.1^{*}$ & 870.9 & 2 & $1 e_{1 g}$ & $9.24^{*}$ & 56.37 & 4 \\
\hline $1 \sigma_{u}^{8}(C 1 s)$ & $291.1^{*}$ & 873.0 & 2 & \multirow{2}{*}{\multicolumn{4}{|c|}{$\mathrm{NH}_{3}$}} \\
\hline $2 \sigma_{g}$ & $23.5^{*}$ & 98.16 & 2 & & & & \\
\hline $2 \sigma_{u}$ & $18.38^{*}$ & 66.12 & 2 & $1 a_{1} \quad\left(\begin{array}{ll}N & 1 s\end{array}\right)$ & $405.6^{*}$ & 1204.1 & 2 \\
\hline $3 \pi_{g}$ & $16.36^{*}$ & 64.50 & 2 & $2 a_{1}$ & $27.77^{*}$ & 98.03 & 2 \\
\hline $1 \pi_{u}$ & $11.40^{*}$ & 57.24 & 4 & $1 e$ & $16.0^{*}$ & 71.33 & 4 \\
\hline & & & & $3 a_{1}$ & $10.88^{*}$ & 84.84 & 2 \\
\hline \multicolumn{4}{|c|}{$\mathrm{C}_{2} \mathrm{H}_{6}$} & \multicolumn{4}{|c|}{$\mathrm{CH}_{4}$} \\
\hline $1 a_{1 g}(C 1 s)$ & $290.5^{*}$ & 871.6 & 2 & $1 a_{1}(C 1 s)$ & $290.7^{*}$ & 871.8 & 2 \\
\hline $1 a_{2 u}(C 1 s)$ & $290.5^{*}$ & 872.0 & 2 & $2 a_{1}$ & $23^{*}$ & 66.68 & 2 \\
\hline $2 a_{1 g}$ & $23.6^{*}$ & 69.16 & 2 & $1 t_{2}$ & $14.35^{*}$ & 51.72 & 6 \\
\hline $2 a_{2 u}$ & $20.16^{*}$ & 67.73 & 2 & & & & \\
\hline $1 e_{u}$ & $15.4^{*}$ & 48.56 & 4 & \multicolumn{4}{|c|}{$\mathrm{C}_{2} \mathrm{H}_{4}$} \\
\hline $3 a_{1 g}$ & $13.5^{*}$ & 65.05 & 2 & $1 a_{g}(C 1 s)$ & $290.9^{*}$ & 871.4 & 2 \\
\hline $1 e_{g}$ & $12.36^{*}$ & 56.26 & 4 & $1 b_{1 u}^{8}(C 1 s)$ & $290.9^{*}$ & 872.4 & 2 \\
\hline
\end{tabular}

are determined, while the angular distribution and energy losses of the scattered particles (primary protons) are integrated over. The angular distribution of fast ejected electrons exhibits a prominent peak, known as the binary peak, and the fractional width of the peak narrows as the electron energy increases. This is a direct consequence of billiard-ball-like collisions between the incident particles and the target electrons. We can again use a simple model of a free electron initially at rest to predict the angle $\theta_{b}$ at which the binary peak will appear (see Appendix B):

$$
\begin{aligned}
\theta_{b} & =\cos ^{-1}\left[\frac{1}{2}\left(M W / m T_{0}\right)^{1 / 2}\right] \quad \text { (proton impact) } \\
& =\cos ^{-1}\left[(W / T)^{1 / 2}\right] \quad \text { (electron impact). }
\end{aligned}
$$

An electron bound in an atom or molecule has its own initial momentum distribution, which will be superimposed on the delta-function angular distribution at $\theta_{b}$. The actual shape of the binary peak, which is known as the Compton profile, will depend on this initial momentum distribution; it will be wide if the initial momentum dis- tribution is broad, and narrow if the target momenta are small compared to the momentum of the ejected electron. The binding energy of an ejected electron is only a rough indication of the initial momentum of the electron because electrons in an orbital that has many nodes have high average momentum but low binding energies. To estimate the width of the peak, one should use the average kinetic energy of a bound electron, as opposed to its binding energy [see Tables $\mathbf{I}(\mathrm{a})$ and $\mathbf{I}(\mathrm{b})$ ].

\section{G. Soft and hard collisions}

Proton-atom collisions can be divided into two qualitatively different types; a soft (or a glancing) collision and a hard (or binary) collision. In a soft collision, momentum transfer is small, the impact parameter is large, and the proton's trajectory hardly deviates from a straight line. Most collisions, particularly for fast protons, are of this type.

In a hard collision, momentum transfer is large with a 
TABLE I(b). (Continued).

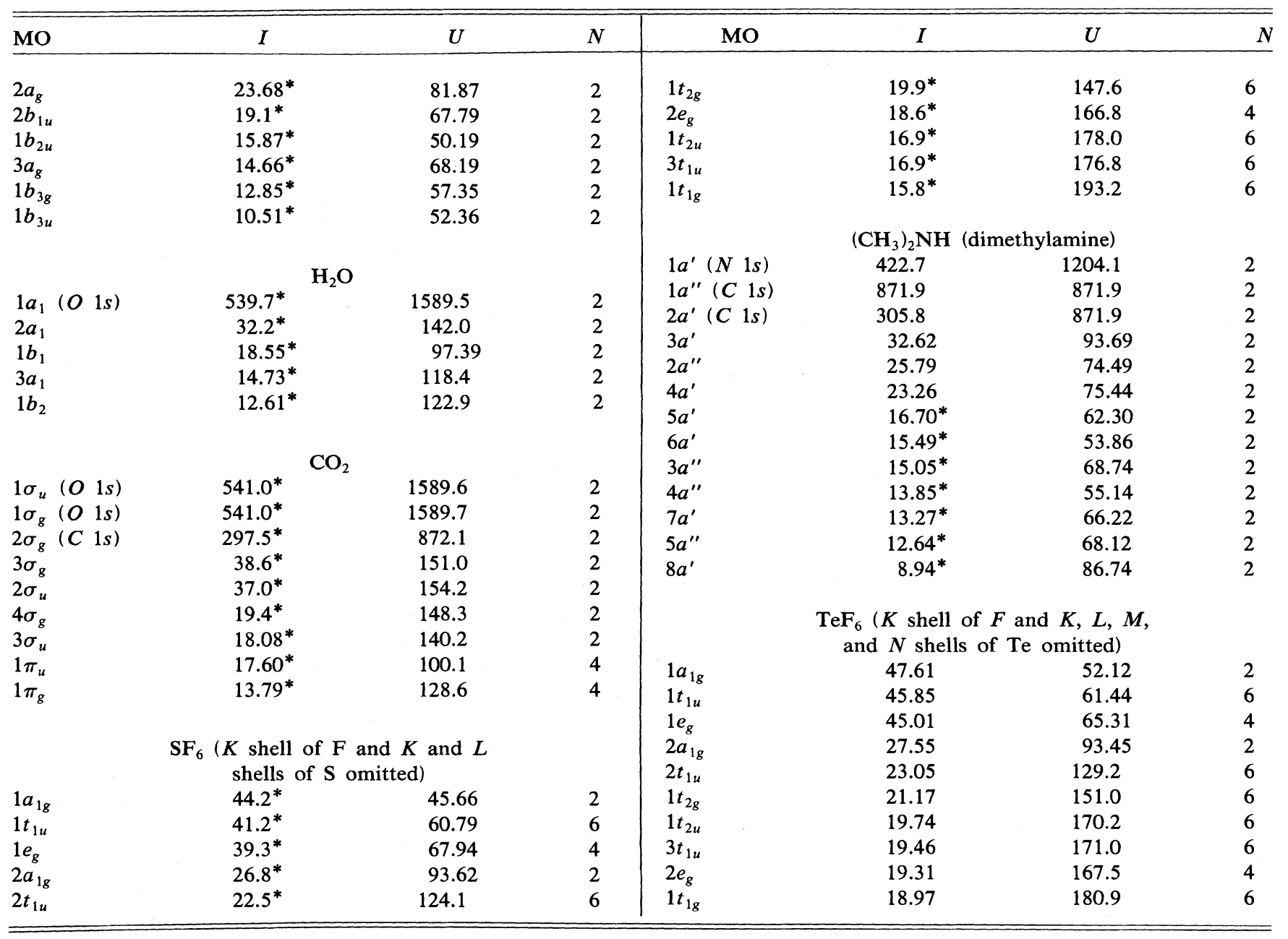

small impact parameter, and the proton's trajectory is significantly altered. The basic characteristics of a hard collision are similar to those of an elastic collision between two billiard balls, in spite of the fact that energy is lost, since the energy loss is small relative to the total energy. Hard collisions correspond to "direct hits" in the classical sense, and for these collisions there is a large energy transfer to the electrons, which then emerge from the collision in a sharp peak (known as the binary peak, see Sec. III.F), which is easily predicted using classical mechanics. Since these collisions are basically classical in nature, they are well described by the Rutherford formula, as described in Sec. III.E. The contribution of hard collisions to a total ionization cross section is more significant for slow incident protons than for fast protons.

\section{H. Optical (zero-momentum-transfer) limit}

In the plane-wave Born approximation, the generalized oscillator strength, which is basically the form factor describing a collision, reduces to the dipole oscillator strength in the limit of zero momentum transfer. As was discussed by Rudd et al. (1985) for the case of total ionization cross sections, this connection between the generalized oscillator strengths and dipole oscillator strength for singly-differential cross sections can be used both in checking the consistency of experimental SDCS's and in formulating semiempirical models for SDCS's (see Sec. IV). Although the zero-momentum-transfer limit is never reached in an inelastic collision, the physically allowed minimum momentum transfer becomes very small for a fast projectile. Hence this dipole contribution is significant for soft collisions with fast protons, eventually dominating the SDCS at incident energies of a few $\mathrm{MeV}$ and above. In the optical limit, collisions with fast protons correspond to the atom absorbing a photon.

A free electron, however, cannot absorb a photon because the total momentum cannot be conserved without a third body (the nucleus in a real atom) to recoil. As a result, classical methods treating atomic electrons as free electrons (even with momentum distributions associated 
with the electrons) - such as those described in Sec. IV - cannot properly account for this dipole contribution and therefore are unreliable for collisions with fast protons.

\section{Semiclassical treatment and the plane-wave Born approximation}

Although an ideal formulation of a collision theory would be based on quantum mechanics, one can treat the trajectory of heavy projectiles classically, while describing the interaction between the projectile and a target atom or molecule using quantum mechanics. This approach is called semiclassical collision theory. The basic assumptions for the validity of the semiclassical theory are (a) that the projectile has high momentum, $k a_{0} \gg 1$, and (b) that the projectile energy is far greater than any interaction potential $V_{\text {int }}$ it will encounter, i.e., $T_{0} \gg V_{\text {int }}$. Under these assumptions, an integrated cross section obtained from a semiclassical theory by integrating over the scattering angle reduces to the integrated cross section derived from the plane-wave Born approximation (Bethe and Jakiw, 1986).

Both assumptions (a) and (b) above are easily satisfied by a slow but heavy projectile such as a proton because of its large mass and the fact that it has a large kinetic energy compared to the usual energy loss involved in an ionizing collision. The normal criterion for validity of the PWBA is $T \gg E$, where $T$ is the energy of an electron with the same speed. For protons, condition (b) can be satisfied although the usual validity condition for the PWBA is not satisfied. This is the reason that the PWBA cross sections for slow ( $<1$ a.u.) protons agree well with experiment, even though this would not be anticipated from the normal PWBA condition.

\section{THEORETICAL TREATMENTS}

The theoretical calculations of singly-differential cross sections for proton-impact ionization fall into three general categories, the classical binary-encounter approximation, the classical-trajectory Monte Carlo approach, and quantum-mechanical methods.

\section{A. Binary-encounter approximation}

The approximation made in this method is to treat the collision as a classical one between the projectile and a single electron in the target; the nucleus and the remaining electrons in the target play no part except that of providing a binding energy for the electron being ejected. In this model the energy transfer $E$ and the energy $W$ of the ejected electron are related by Eq. (12). The justification for using a classical model lies in the fact that differential cross sections (angular distributions) for Coulomb scattering of unlike particles are the same when calculated using either classical physics or quantum mechanics
(Mott and Massey, 1965).

Thomson (1912) utilized the results of the Rutherford scattering theory and derived an expression that may be converted into the Rutherford cross section of Eq. (11). For the Thomson result, the target electron was assumed to be at rest before the collision. This restriction was removed in the work of Williams (1927) and in the more general treatment of Thomas (1927). The results of Thomas (1927) are given for the case of proton impact in a particularly compact form by Vriens (1967). While the binary-encounter-approximation results of Vriens may be evaluated by using a delta-function distribution for the initial momentum of the atomic electron, better results are obtained by using a more realistic distribution of momenta, which must then be integrated over. Rudd et al. (1971) calculated binary-encounter-approximation results using the quantum-mechanical Fock hydrogenic momentum distribution. Some of these results are shown in Fig. 1 , where it is seen that the agreement with experiment is quite good at high primary energies but deteriorates at lower energies. It should be noted that the Fock distribution depends upon a parameter that is the average initial electron velocity for the atomic electron. For the results shown in Fig. 1, the binding energy was used to calculate this average velocity. In some cases, a small improvement may be effected by using Slater's rules (Slater, 1930; Clementi and Raimondi, 1963; Clementi, Raimondi, and Reinhardt, 1967) or some other method to obtain this average velocity.

A simpler form of the binary-encounter theory leads to a singly-differential cross section of the form (Thomas, 1927; Vriens, 1967; Inokuti, 1971)

$$
d \sigma_{\mathrm{BE}} / d W=\left(d \sigma_{R} / d W\right)[1+4 U / 3 E]
$$

$$
\begin{aligned}
& \text { for } E_{\min } \leq E \leq E_{-}, \\
& \begin{aligned}
d \sigma_{\mathrm{BE}} / d W=\left(d \sigma_{R} / d W\right)(U / 6 E)\{ & (4 T / U)^{3 / 2} \\
& \left.+\left[1-(1+E / U)^{1 / 2}\right]^{3}\right\}
\end{aligned}
\end{aligned}
$$

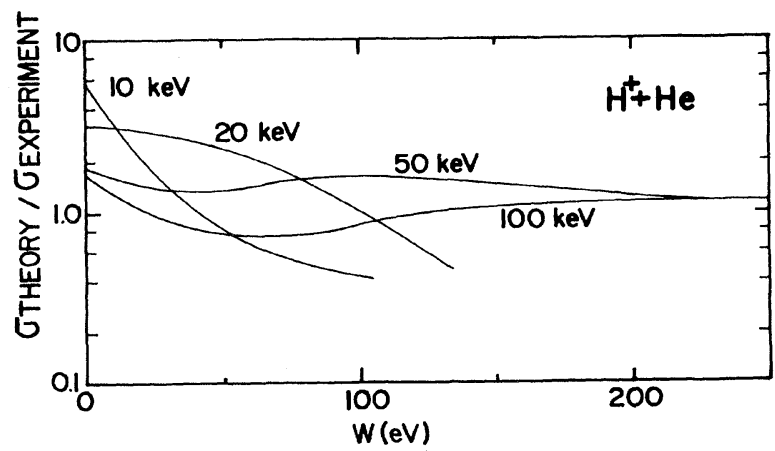

FIG. 1. Comparison of experiment and the binary-encounter approximation for the singly-differential cross sections for ionization of helium by 10 to $100 \mathrm{keV}$ protons. The binaryencounter approximation was obtained by integrating over a Fock distribution of energies for the atomic electron. 
for $E_{-} \leq E \leq E_{+}$, and

$$
d \sigma_{\mathrm{BE}} / d W=0
$$

for $E>E_{+}$, where $U$ is the kinetic energy of the target electron and

$$
E_{ \pm}=4 T \pm 4(T U)^{1 / 2} \text {. }
$$

It is tempting to equate the minimum energy transfer $E_{\min }$ to the ionization threshold I, but the quantummechanical derivation of Eq. (16a) assumes that $U<E$ (Inokuti, 1971). Also, the fact that the target electron must gain energy after a binary collision requires that $T \geq U$. Since most atoms and molecules have a $U$ value of the order of 1-4 atomic units for valence electrons, the minimum $T$ for which Eqs. (16a) and (16b) are valid is of the order of $25-100 \mathrm{keV}$ for incident protons.

Again, the relationship between $E, W$, and $U$ must be redefined for multishell atoms. Binary-encounter theory does not properly account for the logarithmic dependence of ionization cross sections on $T$ or $T_{0}$, which arises from the dipole interaction and dominates ionization cross sections at high incident energies.

\section{B. Classical-trajectory Monte Carlo method}

The binary-encounter approximation discussed in Sec. IV.A treats atomic ionization as a binary collision between the incident proton and the electron to be ionized, without involving the rest of the atom (Gryzinski, 1959, 1965a, 1965b, 1965c; Gerjuoy, 1966; Vriens, 1969; Bonsen and Vriens, 1970). This early model was extended by Abrines and Percival (1966a, 1966b) to incorporate more realistic three-body dynamics. Such a treatment, which has become known as the classical-trajectory Monte Carlo method, has been applied to atomic ionization by Bonsen and Banks (1971), Olson and Salop (1977), Olson (1983), Reinhold and Falcon (1986), Olson et al. (1987), Reinhold et al. (1987), Olson and Gay (1988), and Reinhold and Olson (1989).

In the classical-trajectory Monte Carlo method, the initial state of the ionized electron is represented by a momentum distribution chosen in such a way that it is at least a sensible representation of the momentum distribution one would get from quantum-mechanical wave functions (for the case of scattering from hydrogen, this momentum distribution is exact). The rest of the atom is treated as an inert core represented either by a screened Coulomb potential or by a model potential. In both cases, the independent-electron model (Hansteen and Mosebekk, 1972; McGuire and Weaver, 1977) is used for electron ejection from many-electron atoms. In the independent-electron model, correlations between the atomic electrons are neglected and it is assumed that each electron can be regarded as being independent.

The calculation proceeds by randomly choosing the phase-space coordinates (i.e., position and momentum) for the atomic electron, using the above momentum distribution, and by randomly choosing an impact parame- ter for the incident proton. The evolution of the collision system is then found by integrating the classical equations of motion for the three-body system of projectile, target electron, and atomic core from a large initial projectile-target separation to a large final separation. The relative energies and positions of the particles are then used to determine scattering angles and which reaction, if any, has occurred. This procedure is repeated a large number $\left(\sim 10^{5}\right)$ of times. After a sufficiently large ensemble of projectile-target configurations has been sampled, the differential cross sections are determined. Although there have been numerous classical-trajectory Monte Carlo calculations performed for various scattering processes, in the case of singly-differential cross sections for proton-impact ionization, the most complete calculations reported to date are for atomic hydrogen (Olson, 1983; Reinhold et al., 1987) and helium targets (Abrines and Percival, 1966; Reinhold and Falcon, 1986; Olson et al., 1987; Olson and Gay, 1988; Reinhold and Olson, 1989).

\section{Quantum-mechanical methods}

The quantum-mechanical treatments for atomic ionization by proton impact fall into three different classes: (1) those which treat the electron-target interaction as a strong interaction and the electron-projectile interaction as a weak interaction; (2) those which treat the electrontarget interaction as strong and the electron-projectile interaction as strong for the incoming proton and as weak for the outgoing proton; and (3) those which treat both the electron-target and electron-projectile interactions as strong interactions. Clearly, treatments of type (1) will be valid when the outgoing electron and projectile have significantly different speeds or outgoing directions. Treatments of type (3) will be required if the ejected electron and projectile have similar speeds and are leaving the collision region in similar directions.

\section{Weak initial- and final-state electron-projectile interactions}

If the electron-projectile interaction is weak, this interaction may be treated perturbatively. Theoretically, this is accomplished by expressing the wave function for the electron as a single-center wave function whose origin is located at the target nucleus. The interaction between the electron and projectile is ignored in the formation of the electron wave function and, as a result, this interaction appears only in the interaction potential in the transition matrix element, which is treated perturbatively.

The calculations that have been performed within this framework have assumed either that the projectile is undeflected and moves in a classical trajectory or that the projectile wave function can be expressed as a plane wave. The former approach of assigning a classical trajectory to the heavy projectile and treating the rest of the problem quantum-mechanically is known as the semiclas- 
sical approximation (Bang and Hansteen, 1959), and the latter approach is called the plane-wave Born approximation (PWBA). Although there has been considerable use of the semiclassical approximation for total cross sections for inner-shell ionization (Madison and Merzbacher, 1975), there have been relatively limited applications to singly-differential cross sections for proton impact (Mukoyama et al., 1985). It can be shown that the semiclassical approximation and PWBA yield identical results for energy distributions of the ejected electron in heavyparticle ionization (Bethe and Jackiw, 1968; Madison and Merzbacher, 1975). Most of the work germane to this review has been performed within the PWBA.

The PWBA (or standard straight-line trajectory semiclassical approximation) will certainly be invalid if the proton is deflected through a large angle $\left(>1^{\circ}\right)$. However, for the types of experiment germane to this review, this is a good approximation, since the results are integrated over proton scattering angles, and the major contributions to the cross section come from very small proton scattering angles. The distinction between different calculations of this type then lies in the treatment of the ejected-electron wave function. For the calculations that have typically been labeled PWBA's, the ejected-electron wave function is approximated as a plane wave, and for the distorted-wave Born approximation (DWBA), the ejected-electron wave function is calculated as an eigenfunction of some model potential representing the residual target ion.

In the standard theoretical development, a partialwave expansion is made for the final-state ejectedelectron wave function. The radial part of this wave function $\chi_{l}(k, r)$ for a partial wave with angular momentum $l$ for the ejected electron is a solution of the Schrödinger equation,

$$
\left[\frac{d^{2}}{d r^{2}}-\frac{l(l+1)}{r^{2}}-V(r)+k^{2}\right] \chi_{l}(k, r)=0,
$$

where $V(r)$ is a (spherically symmetric) potential representing the residual ion. Over the years, there have been various choices made for this potential ranging from no potential (PWBA) to a Coulomb potential for some effective charge or a numerical potential obtained from Hartree-Fock wave functions for the atom in question. In terms of nomenclature, the effective-Coulomb-field choice would appropriately be called the Coulomb-Born approximation and the numerical Hartree-Fock-potential choice would be called the DWBA. The difference between the Coulomb-Born and DWBA is most pronounced in the doubly-differential cross section at large scattering angles. In general, it is best to use a DWBA even for the singly-differential cross section, since significant differences between the two approaches can be observed.

The DWBA approach was discussed by Rudd et al. (1985), and the formulas for the SDCS are contained therein; we shall not repeat that presentation here. The interested reader is referred to that review for a discus- sion of the philosophy of the Born approximation, a description of the calculation of self-consistent-field wave functions and potentials, orthogonality requirements, different types of DWBA calculations, conditions for validity of the DWBA, the numerical problems associated with a DWBA calculation, the connection between incident protons and electrons, the connection between proton- and photon-impact ionization, and the lowenergy behavior of the cross sections.

\section{Strong initial-state interactions and weak final-state interactions}

The Glauber approximation (Glauber, 1959) is designed to include a strong electron-projectile interaction in the initial state. It is very similar to the Born approximation except that instead of using a plane wave for the initial projectile state, it uses an eikonal approximation for the exact wave function. In the eikonal approximation, the initial state is a plane wave modified by a phase factor which is proportional to the average of the perturbing potential over the trajectory of the particle. When used in the scattering amplitude, it can be seen that this eikonal wave function generates the first Born amplitude exactly and higher Born amplitudes approximately. The Glauber approximation is obtained from the eikonal amplitude by choosing a particular trajectory for the projectile. For the final state of the system, the typical Glauber calculation would use the same type of wave function as the typical Born calculation. Consequently, in terms of the strengths of interactions, the Glauber approximation assumes a stronger initial-state electronprojectile interaction than does the Born and the same weak final-state electron-projectile interaction. As a result, in similarity to the Born approximation, the Glauber approximation would not be expected to be valid for those cases in which the electron is ejected near the projectile and with the same speed.

The Glauber approximation was first applied to atomic physics problems by Franco (1968). Golden and McGuire (1974,1977) and McGuire (1982) applied the Glauber approach to the problem of atomic ionization by heavy projectiles and found that it yielded accurate total ionization cross sections at high energy. Limited results have been reported for the singly-differential cross section, which is of interest here.

\section{Strong electron-projectile interactions}

\section{a. Continuum-distorted-wave method}

The PWBA, DWBA, or Glauber methods would not be expected to be valid when the ejected electron leaves the collision with approximately the same velocity as the projectile proton, since there would then be a strong final-state interaction that is treated only to first order in the perturbation expansion. When this happens, the at- 
tractive nature of this force will cause an enhancement of the electrons being scattered in the direction of the proton. This effect has been observed in double-differential cross-section measurements and has been called both charge transfer to the continuum and, more recently, electron capture to the continuum, as was discussed in Sec. III.D.

Over the last several years, there has been a significant effort directed towards the development of theoretical models that take into account both the final-state electron-target interaction and the final-state electronprojectile interaction in a symmetrical manner ( $R y u f u k u$, 1982). Such treatments by definition require a two-center final-state wave function, since one part of the electronic wave function must be centered on the target and another part on the projectile. A review of the experimental observation and theoretical treatment of two-center effects has recently been given by Fainstein et al. (1991). One theoretical model that has been successful in its treatment of two-center effects is the "continuumdistorted-wave" approach. Cheshire (1964) developed this model for charge-exchange scattering, and Belkić (1978) applied it to atomic ionization by heavy particles. The primary characteristic of a continuum-distortedwave calculation is that all two-particle Coulomb interactions are contained explicitly in the initial- and final-state wave functions. As a result, the final-state wave function in the continuum-distorted-wave approach is represented as a product of a Coulomb wave function for the electron-target subsystem, a Coulomb wave function for the electron-projectile subsystem, and a Coulomb wave function for the projectile-target subsystem. The initialstate wave function is expressed in a similar fashion except that the electron-target wave function is an initial bound state for this case.

A modified version of the continuum distorted wave was proposed by Garibotti and Miraglia (1980), who used the final state described above but assumed a weak electron-projectile interaction in the incident channel. As a result, the initial-state wave function in that work was chosen to be a product of a plane wave for the projectile and a bound state for the electron (the same type of choice is made in the PWBA). Previously, however, Belkić (1978) found that it was important in general to assume a strong electron-projectile interaction in the initial state also. Crothers and McCann (1983) developed the continuum-distorted-wave method within the framework of the semiclassical impact-parameter timedependent approach. For heavy projectiles it is expected that the impact-parameter method should be essentially equivalent to full quantal methods. In fact, Belkic (1978) demonstrated the equivalence for forward scattering and very massive targets. In the Crothers and McCann (1983) work, the final state is treated in the standard continuum-distorted-wave fashion and the initial state is represented as an eikonal approximation to the initialstate continuum distorted wave. Using the eikonal initial state has proven to be very successful, and this type of calculation is now called the CDW-EIS (continuumdistorted-wave eikonal-initial-state) approximation.

Fainstein et al. (1988a) extended the CDW-EIS approximation from the case of ionization of a monoelectronic target by a bare ion to the multielectronic case in which there is a single active electron, and have successfully applied it to the problem of proton and antiproton ionization of heavier atoms (Fainstein et al., 1988b, 1989a, 1989b). The primary interest and motivation for most of the continuum-distorted-wave work has been the desire to understand and interpret experimental DDCS results, but some SDCS results have also been reported (Fainstein et al., 1988, 1989b; Crothers and McCann, 1983; Belkić, 1978).

\section{b. Other methods for strong electron-projectile interactions}

In addition to the continuum-distorted-wave method, there are other theoretical approaches that contain a nonperturbative strong electron-projectile interaction. This interaction is included exactly in the classicaltrajectory Monte Carlo method described in Sec. IV.B. Two other approaches also deserve mention-the coupled-channels approach and direct integration of the time-dependent Schrödinger equation.

Shakeshaft (1978) performed a coupled-state calculation for proton-hydrogen scattering that used wave functions centered on both the target and the projectile. In this work, the standard time-dependent impactparameter coupled-state method was used and 35 basis functions centered on both the projectile and the target were used in the expansion of the electron wave function. Singly-differential cross sections were not reported in that work, but it was noted that for energies less than about $75 \mathrm{keV}$, charge-transfer-to-the-continuum effects are significant, to the extent that theories neglecting them, such as the PWBA or DWBA, should be inadequate below that energy. There have been numerous other coupled-channels calculations for atomic ionization by heavy projectiles, but the focus of those works is outside the scope of this review (see, for example, Reading et al., 1979, 1981; Reading and Ford, 1979, 1987; Janev and Presnyakov, 1980; Ford et al., 1981; Fritsch and Lin, 1983; Paul and Obermann, 1983; Winter and Lin, 1984).

The last method we shall mention, which automatically includes a strong electron-projectile interaction, is the direct integration of the time-dependent Schrödinger equation by Bottcher (1982). In this work, it was assumed that the projectile was a bare nucleus moving with a uniform velocity in a straight line. The time-dependent Schrödinger equation was then propagated from the initial state to the final state and cross sections for excitation, capture into bound states, direct ionization, and charge transfer to the continuum were determined from the resulting final states. Although only total cross sections were reported in this work, it does represent an interesting theoretical development. 


\section{Comparison of experiment and theory}

At present, $a b$ initio theories are limited in their validity to certain ranges of proton energies and/or to certain targets. Similarly, available experimental data are limited in the ranges of ejected-electron energies and/or incident-proton energies. Often the missing parts in the experimental data are supplemented using semiempirical models. Section VI of this review will show that the experimental single-differential cross-section data can be expressed in terms of analytic formulae with targetdependent adjustable parameters. The recommended experimental data for $\mathrm{He}$ expressed in this parametrized form are compared with the DWBA calculations described by Rudd et al. (1985) and the classical-trajectory Monte Carlo calculations of Schultz and Reinhold (1989) at incident proton energies of $100 \mathrm{keV}, 400 \mathrm{keV}$, and 1 $\mathrm{MeV}$ in Figs. 2-4. As was noted earlier, Shakeshaft (1978) demonstrated that the effects of charge transfer to the continuum start to become significant below $75 \mathrm{keV}$, so the DWBA would not be expected to be valid for much lower energies, since charge-transfer-to-thecontinuum effects are included only to first order in a perturbation expansion. In general, both theories give a reasonably good representation of the experimental data. At $1 \mathrm{MeV}$ the DWBA is in very good agreement with experiment over the entire energy range of the ejected electron. At this energy, the classical-trajectory Monte Carlo result is in excellent agreement with experiment for the higher electron energies but falls below the experiment for the lower energies, where the cross sections are larger.

This problem with the slow electrons is a manifestation of the fact that the classical calculations give an asymptotic proton energy behavior of $T_{0}^{-1}$ instead of the quantum-mechanical $T_{0}^{-1} \ln T_{0}$ behavior, a dependence that has been well verified experimentally. The failure of the classical-trajectory Monte Carlo approach for large $T_{0}$ is a direct result of the fact that the classical calcula-

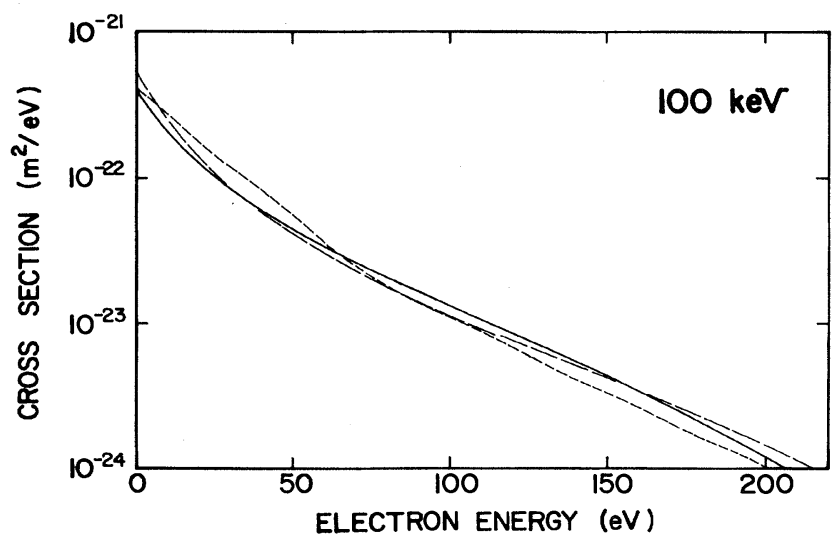

FIG. 2. Singly-differential cross sections for ionization of helium by $100-\mathrm{keV}$ proton. The solid curve represents the best fit to experimental data; long-dashed curve, distorted-wave Born approximation; short-dashed curve, classical-trajectory Monte Carlo.

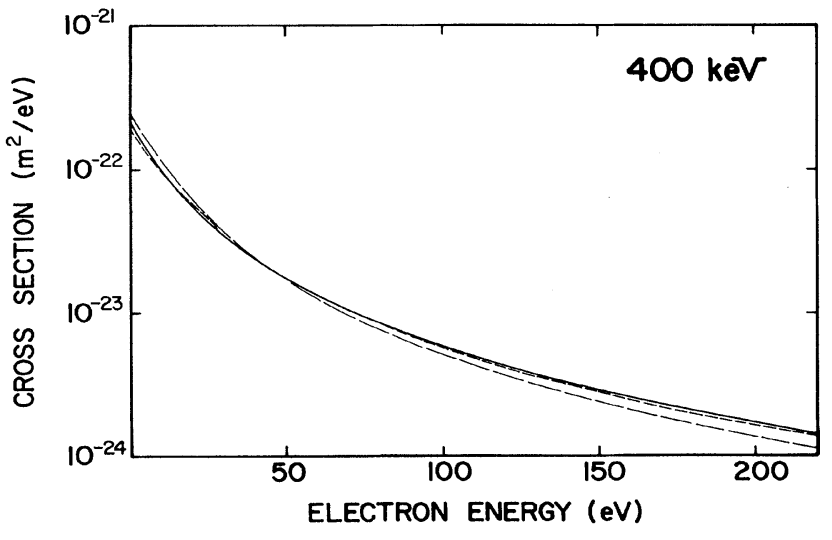

FIG. 3. Same as Fig. 2 except for $400-\mathrm{keV}$ proton.

tions do not contain the dipole contribution to the scattering process, which is the source of the $T_{0}^{-1} \ln T_{0}$ energy dependence. While the dipole contribution is significant for large $T_{0}$, nondipole terms are larger than the dipole term at intermediate $T_{0}$, which explains why the classical calculations are better in this energy region. At $400 \mathrm{keV}$ the classical-trajectory Monte Carlo result is in better agreement with experiment than the DWBA. Here the classical-trajectory Monte Carlo calculation is in excellent agreement with experiment over the entire secondary-electron energy range, while the DWBA is somewhat too large for low electron energies. At the lowest incident energy considered, $100 \mathrm{keV}$, the DWBA is again in somewhat better agreement with experiment than the classical-trajectory Monte Carlo approach. We note, however, that these Monte Carlo calculations were performed using a screened Coulomb potential to represent the target core, and that Reinhold and Falcon (1986) have shown that the use of a model potential interaction brings the classical-trajectory Monte Carlo result into better agreement with experiment at lower secondary-electron energies.

In general, the DWBA will be in very good agreement with experiment for high proton energies $\left(T_{0} \gtrsim 1 \mathrm{MeV}\right)$, in reasonably good agreement for intermediate energies

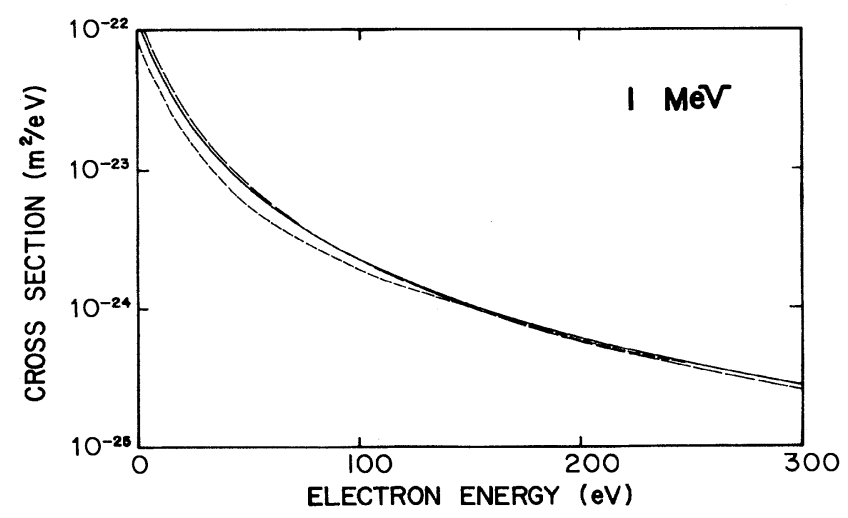

FIG. 4. Same as Fig. 2 except for 1-MeV proton. 
(100 keV $\lesssim T_{0} \lesssim 1 \mathrm{MeV}$ ), and in increasingly poor agreement with experiment for lower proton energies. For the comparisons we made for $\mathrm{He}$, it was found that the DWBA was within $10 \%$ of experiment at $1 \mathrm{MeV}$, within about $20 \%$ of experiment in the $100-\mathrm{keV}$-to-1-MeV range, and within $50-100 \%$ of experiment by $40 \mathrm{keV}$. The classical-trajectory Monte Carlo method, on the other hand, works best in the intermediate-energy range, with decreasing validity both for the higher and lower energies. For the cases we considered, the Monte Carlo calculation was within $10 \%$ of experiment at the intermediate energy $(400 \mathrm{keV})$, with the difference increasing to the $30-40 \%$ range for both the lower and higher proton energies. For atoms heavier than helium, theoretical results start to depend strongly on the quality of the atomic wave functions used for the outer electrons and other multielectron effects. As a result, PWBA calculations using Hartree-Fock wave functions tend to overestimate cross sections with increasing atomic number.

\section{CONSISTENCY CHECKS}

Most measurements of singly-differential cross sections are indirect, i.e., SDCS's are deduced by integrating DDCS's over the electron-ejection angle. Moreover, a SCDS must be consistent with the corresponding total ionization cross section when the SDCS is integrated over the electron energy.

The measurement of a DDCS for an atom or molecule involves many continuous variables, such as the incident proton energy, the secondary electron energy, and the electron ejection angle. The data thus tend to be incomplete if one wishes to perform integrations to obtain a SDCS. Therefore it is important to identify methods for checking the consistency of existing data and to supplement missing parts by comparison with other available information.

For instance, if Eq. (4) does not agree with a known value of the total ionization cross section, it is desirable to know if some part of $d^{2} \sigma / d W d \Omega$ may be causing the discrepancy rather than simply to renormalize $d^{2} \sigma / d W d \Omega$ uniformly. One can also interpolate and extrapolate experimental $d \sigma / d W$ in both $W$ and $T_{0}$ if it can be established that the observed $d \sigma / d W$ exhibits qualitative trends expected from theoretical considerations.

In this section, we discuss several powerful consistency checks for identifying qualitative trends in $d \sigma / d W$. Although these trends are based on the PWBA and are hence valid only for fast incident protons $\left(T_{0}>300 \mathrm{keV}\right)$, they are nevertheless very useful in detecting problems in experimental data and in supplementing missing parts in such data.

\section{A. Bethe theory}

Bethe (1930) derived the basic framework of the Born approximation for ionization and excitation of atoms and molecules by charged-particle impact. In this monumental work, he introduced the generalized oscillator strength, which is basically a collision form factor that reduces to the dipole oscillator strength $f$ in the limit of zero-momentum transfer and that satisfies a sum rule similar to that for $f$. The generalized oscillator strength for a given transition is a function of momentum transfer, which is determined by the angular distribution of the scattered particle. Bethe showed further that the cross section to excite an atom to state $n, \sigma_{n}$, depends on $T=(m / M) T_{0}$, i.e., on the velocity of the incident particle. The cross section integrated over the scattering angle of the incident particle is conveniently expanded as a function of $T$ :

$$
\sigma_{n}=\left(4 \pi a_{0}^{2} R / T\right)\left[a_{n} \ln (T / R)+b_{n}+c_{n} R / T+\cdots\right],
$$

where $a_{n}, b_{n}$, and $c_{n}$ are constants that depend on the initial and final states of the target but not on the projectile energy. Note that $T$, not $T_{0}$, is used in Eq. (18) regardless of the projectile mass. In particular, $a_{n}$ has a simple relationship to the dipole oscillator strength $f_{n}$ and excitation energy $E_{n}$ :

$$
a_{n}=f_{n} R / E_{n} .
$$

For the singly-differential cross section, Eqs. (18) and (19) become

$$
\begin{gathered}
d \sigma / d W=\left(4 \pi a_{0}^{2} R / T\right)[a(E) \ln (T / R)+b(E) \\
+c(E) R / T+\cdots], \\
a(E)=(d f / d E) /(E / R),
\end{gathered}
$$

and $E=W+I$ is now the excitation energy to a specific continuum final state. Note that the singly-differential cross sections discussed in this review are differential in the energy of the ejected electron and are expressed in units of $($ area $) \times(\text { energy })^{-1}$. This implies that $a(E)$, $b(E)$, and $c(E)$ have the dimension of (energy) ${ }^{-1}$.

Another important conclusion from Bethe's work is that the essential part of the PWBA is the first two terms in the square brackets of Eqs. (18) and (20). Moreover, these two terms are the same for incident protons and electrons of equal speeds. Other physical effects not included in the Bethe theory - such as the distortion of the projectile wave function and the polarization of the target charge distribution - may introduce a $T$ dependence that can be more important than the $T^{-1}$ terms associated with $c_{n}$ or $c(E)$ for fast projectiles. Differential cross sections for protons with $T_{0}<100 \mathrm{keV}$, for example, may be altered significantly by charge transfer to the continuum, a process not included in the Bethe theory. Experimental data for neutral atoms indicate that the $c(E)$ term for ionization is small for incident protons of a few hundred $\mathrm{keV}$ and higher.

For a multishell atom or molecule, Eqs. (20) and (21) must be generalized to accommodate electrons ejected with the same kinetic energy $W$ but from different shells. 
In the usual experimental situation, where the initial electron shell is unknown and contributions from individual shells must be summed over, Eq. (20) is thus replaced by

$$
\begin{array}{r}
d \sigma / d W=\left(4 \pi a_{0}^{2} R / T\right) \Sigma_{j}\left[a_{j}\left(E_{j}\right) \ln (T / R)+b_{j}\left(E_{j}\right)\right. \\
\left.+c_{j}\left(E_{j}\right) R / T+\cdots\right]
\end{array}
$$

with

$$
E_{j}=W+I_{j},
$$

where $I_{j}$ is the binding energy of the $j$ th orbital, and

$$
a_{j}\left(E_{j}\right)=\left(d f / d E_{j}\right) /\left(E_{j} / R\right) .
$$

In principle, $a_{j}\left(E_{j}\right), b_{j}\left(E_{j}\right)$, and $c_{j}\left(E_{j}\right)$ can be calculated if the corresponding wave functions are known (Kim and Inokuti, 1971), but a systematic study of $a_{j}\left(E_{j}\right)$ and $b_{j}\left(E_{j}\right)$ has been carried out only for the ionization of the hydrogen atom (Inokuti, 1971).

The $T$ dependence described in Eq. (22) is valid only when $T$ is larger than the average (orbital) kinetic energies of the bound electrons being ionized [see Tables I(a) and $I(b)$ ]. For multishell atoms, the average kinetic energies of the valence and core orbitals are so different that the $T^{-1} \ln T$ dependence predicted by the PWBA may be valid for the ionization of the valence electrons but not for the core electrons. Over most of the range of secondary energies, the contributions from inner shells to $d \sigma / d W$ are small compared to those from outer shells. The $T$ dependence predicted by the PWBA is often observed in experimental cross sections with incident proton energies of a few hundred $\mathrm{keV}$.

\section{B. Platzman plots}

The Rutherford cross section, Eq. (13), is the singlydifferential cross section for a single bound electron. Hence, if we divide the SDCS by the "modified" Rutherford cross section, the result can be interpreted as the effective number of electrons in the target atom or molecule which participate in the ionizing collision of interest. This is the underlying idea for the Platzman plot (Miller and Platzman, 1957). In this plot, the ratio $Y$ of the actual SDCS to the Rutherford cross section [Eq. (13)] is plotted as a function of $R / E$,

$$
\begin{aligned}
Y & =[d \sigma / d W]_{\text {actual }} /\left[d \sigma_{R} / d W\right] \\
& =\frac{T}{4 \pi a_{0}^{2}} \frac{E^{2}}{R^{2}}[d \sigma / d W]_{\text {actual }} .
\end{aligned}
$$

The choice of $R / E$ as the abscissa makes the area under the Platzman plot proportional to the total ionization cross section:

$$
\begin{aligned}
\sigma_{\text {ion }} & =\int_{0}^{W_{\max }}(d \sigma / d W) d W \\
& =\frac{4 \pi a_{0}^{2} R}{T} \int_{R / E_{\max }}^{R / I_{1}} Y d(R / E),
\end{aligned}
$$

where $I_{1}$ is the binding energy of the outermost shell and
$E_{\max }=W_{\max }+I_{1}$.

As was discussed in Sec. III.C, $W_{\max }$ for a collision with a free electron is $4 T=4(m / M) T_{0}$. For a bound electron, however, $W_{\max } \gg 4 T$, since the ion core can recoil and impart extra momentum to the electron. Therefore the lower limit of the integral in Eq. (26) is practically zero for protons of a few hundred $\mathrm{keV}$ and above. We present an example of a Platzman plot for the ionization of Ar by $1 \mathrm{MeV}$ protons in Fig. 5. The matching continuum dipole function, $E(d f / d E)$, is plotted in Fig. 6.

The Platzman plot is a powerful tool for analyzing and identifying distinct features in the SDCS (Kim, 1975a, 1975b, 1975c; 1983). For instance, the dominance of the dipole term in the proton-impact data is evident in Fig. 5. The dip near $R / E \approx 0.3$ in Fig. 6 is known as the Cooper minimum (Fano and Cooper, 1968), and the sharp peak near $R / E \approx 0.07$ in Fig. 5 includes peaks arising from the LMM Auger electrons.

By integrating the area under the SDCS curve from the threshold $(R / E \approx 0.87$ ) to $R / E=0$, one can verify the normalization of the proton-impact data, which, in this example, falls within $10 \%$ of the total ionization cross section recommended by Rudd et al. (1985). If we assume that only the $M$-shell electrons participate in ionizing collisions, then the height of the singly-differential cross section near $R / E \approx 0.3$ should be 8 , since the dipole interaction essentially vanishes here, and hence the ordinate in the Platzman plot should equal the number of free electrons in the participating shell. In reality, the SDCS in Fig. 5 has $Y \approx 10$, indicating that there are addi-

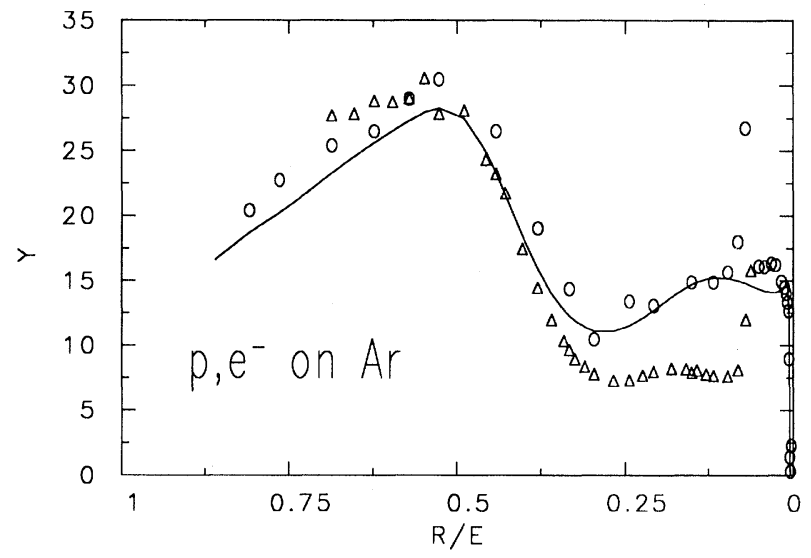

FIG. 5. Platzman plots of proton- and electron-impact singlydifferential cross sections for Ar. The ordinate is the ratio of the SDCS to the Rutherford cross section [see Eq. (25)], and the abscissa is the inverse of the energy transfer, $E=W+I_{1}$ [see Eq. (23)], where $W$ is the secondary-electron energy and $I_{1}$ is the first ionization potential ( $R$ is the rydberg): $0,1-\mathrm{MeV}$ ( $T=545 \mathrm{eV}$ ) proton-impact data of Toburen et al. (1978); $\triangle$, 500-eV electron-impact data of Opal et al. (1972). The solid curve is the recommended SDCS based on Kim's model, discussed in Sec. VI.C. The sharp peaks in the proton- and electron-impact data at $R / E \approx 0.06$ are due to LMM Auger electrons. 


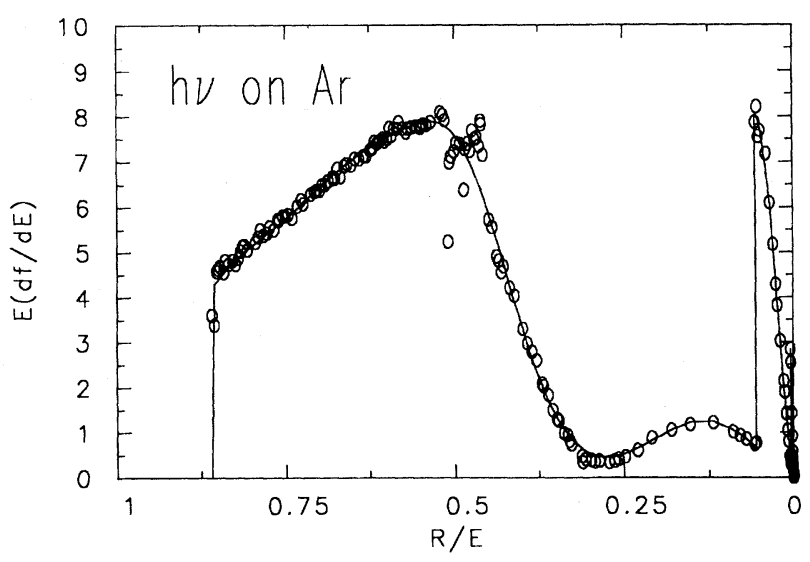

FIG. 6. The dipole function $E(d f / d E)$ of $\mathrm{Ar}$ as a function of the inverse of the photon energy $(E)$ in rydbergs $(R): O$, experimental data compiled by Berkowitz (1979); solid curve, a fit to smoothed experimental data using Gaussian functions and power series (see Sec. VI.C). The structure near $R / E=0.5$ arises from the $3 s-n p$ window resonances, the dip near $R / E=0.3$ is the Cooper minimum, and the sharp rise near $R / E=0.05$ is the onset of the $L$-shell ionization. The onset of the $K$-shell ionization appears as another peak near $R / E=0$.

tional electrons ejected from the $L$ shell and that there are contributions due to the fact that the target electrons are not free but bound with their own momentum distributions, described by the binary-encounter term.

\section{Comparison with electron-impact data}

As was mentioned in Sec. V.A, the leading terms of proton- and electron-impact cross sections are equal when both projectiles are fast and have equal speed. This equality, which is one of the most significant conclusions of the PWBA, has been observed in numerous examples of proton- and electron-impact ionization and has served as a consistency check between experimental data sets.

The actual range of $T$ for which this resemblance holds depends on the target. For targets with simple shell structures, such as $\mathrm{H}, \mathrm{He}$, and $\mathrm{H}_{2}$, the singly-differential cross sections for electron and proton impact agree to better than $10 \%$ for $T>1 \mathrm{keV}$, while much higher $T$ is needed for targets with inner shells. Proton-impact $(T=545 \mathrm{eV})$ and electron-impact $(T=500 \mathrm{eV})$ SDCS's of Ar are presented in Fig. 5, where the resemblance predicted by the PWBA is evident, though the $T$ values are too low to expect detailed matching between them. In general, proton-impact ionization cross sections are larger than the corresponding electron-impact data with the same projectile speed, for the reasons discussed in Sec. III, but the difference diminishes as $T$ increases.

Since the incident-proton energy, $T_{0}=(M / m) T$, is almost 2000 times that of an electron with equal velocity, a proton can eject secondary electrons of much higher kinetic energies than those ejected by an incident electron with energy $T$. In this respect, the resemblance predicted by the PWBA should apply to global properties rather than to local details.

\section{Comparison with photoionization data}

Another prediction of the PWBA is that the leading term in $d \sigma / d W$ at higher energies is the logarithmic term in Eq. (22). This term represents contributions from soft collisions (Sec. III.G) and is directly associated with the continuum oscillator strength $d f / d E$, which can be deduced from photoionization cross sections. As will be shown in the following section, the dipole function $a(E)$ defined in Eq. (24) is distinct from one target to another, and its main features remain clearly discernible in the singly-differential cross section even at lower $T$, where the Bethe formula defined in Eq. (22) is inadequate.

To be useful for consistency checks as well as for modeling of proton- and electron-impact SDCS's, photoionization cross sections for individual shells are needed. Photoionization cross sections usually have energy resolutions far better than those necessary for modeling SDCS's. Coincidence measurements in electron energyloss or photoelectron spectra in which the energy transfer and the kinetic energy of ejected electrons are identified are needed for this type of consistency check.

\section{E. Comparison with total ionization cross sections}

Deducing accurate total ionization cross sections, $\sigma$ [Eq. (4)], from experimental doubly-differential cross sections is not straightforward, since experimental DDCS's often do not cover all ranges of $\theta$ and $W$ for which $d^{2} \sigma / d W d \Omega$ contributes significantly to $\sigma_{-}$. In particular, contributions to $\sigma_{\text {_ }}$ from slow ejected electrons are very important. In most cases, about two-thirds of $\sigma_{-}$ comes from $d \sigma / d W$ for $W$ less than the first ionization potential, yet the experimental cross sections for $W<10$ $\mathrm{eV}$ are often unreliable or unavailable.

This low-energy region is where the Platzman plot, in combination with photoionization data, can play a crucial role in providing the missing information needed to perform the integration over $W$ such that $\sigma_{-}$is consistent with the total ionization cross sections measured directly. In fact, one can normalize $d \sigma / d W$ to a known value of $\sigma_{-}$, even if part of the $d \sigma / d W$ is missing, by using the Platzman plot and known values of $d f / d E$. Examples are given in the next section on semiempirical models. The solid curve in Fig. 5 illustrates a Platzman plot in which the area under the curve has been normalized to the total ionization cross section.

\section{F. Relationship with stopping cross sections}

A frequently used quantity in radiation physics is the "W value," which is the average energy needed to generate an ion. This " $W$ value" is usually determined by di- 
viding the energy loss of a projectile in a medium by the total number of ions it generated-a gross measure of how difficult it is to ionize the medium. The $W$ value of a target is higher than its lowest ionization potential because (a) some of the energy deposited by the projectile is used in discrete excitations without producing any ions and (b) the energy lost by the projectile in ionizing collisions normally exceeds the lowest ionization potential due to the kinetic energy gained by the ejected electron. Fast ejected electrons can also ionize other targets in the medium and produce additional secondary electrons through successive inelastic collisions until their kinetic energy drops below the lowest ionization potential.

The stopping cross section for ionization, $\sigma_{\text {st }}$ [Eq. (6)], accounts for ionizing collisions but not for discrete excitations. The total stopping cross section, however, must include both discrete and continuum excitations (Inokuti, 1971). The $\sigma_{\text {st }}$ defined by Eq. (6), therefore, is a lower bound for the total stopping cross section and constitutes 80-90\% of it (Wilson, 1972). The $\sigma_{\text {st }}$ is also the dominant component in the theoretical determination of the $W$ value, although other factors, such as excitations by the incident proton and subsequent collisions by secondary electrons, must also be taken into consideration.

\section{SEMIEMPIRICAL MODELS}

With the exception of the work of Park et al., all experiments to establish differential ionization cross sections by proton impact have measured angular distributions of ejected electrons as functions of the electron energy. Then, the cross sections were integrated over angle to deduce the singly-differential cross section. Although it is difficult to measure electrons ejected in the extreme forward and backward directions, the integrated cross sections are not sensitive to these angles, since the solidangle element $\sin \theta d \theta d \phi$ reduces the contributions from the extreme angles. If one wishes to obtain total ionization cross sections, a more serious problem presented by existing experimental data stems from the measurement of slow electrons $(W \leq 10 \mathrm{eV})$. Measurements are difficult and often unreliable for slow electrons (see Secs. VII and VIII), while their contributions to the total ionization cross section are very important (20-40\%). Semiempirical models can successfully supplement missing parts of SDCS's and provide consistency checks between SDCS's and total ionization cross sections.

Using the close relationship between the optical dipole oscillator strengths and the Bethe cross section (see Sec. V.A), Kim has shown (Kim, 1975b, 1975d, 1976) that SDCS's for proton-impact ionization can be expressed qualitatively as the sum of a dipole term and the Rutherford cross section. This dipole term is not the same as the $a(E)$ defined by Eq. (21), but rather is scaled by a function of $E$ that depends on the target. This qualitative behavior is best understood through the Platzman plot discussed in Sec. V.B. Some of the semiempirical models presented below take advantage of these qualitative features in the SDCS. The Bethe cross section, however, is based on the PWBA, which is valid for fast protons, and hence a model based on the Bethe theory would eventually fail for slow protons.

At present, there is no single model that accurately reproduces SDCS's for all incident-proton and ejectedelectron energies. Some models are capable of providing accurate and detailed SDCS's in a limited range of proton and/or electron energies. Other models can be used for a wide range of proton and electron energies but do not reproduce the details of SDCS's very well.

\section{A. Miller's model}

In Miller's model (Miller et al., 1987, and references therein), the $b_{j}\left(E_{j}\right)$ function in Eq. (22) is empirically determined by subtracting the dipole contribution, $a_{j}\left(E_{j}\right) \ln \left(4 T R / E_{j}^{2}\right)$, from experimental singly-differential cross sections at sufficiently high $T$ that $c_{j}\left(E_{j}\right)$ and other $T$-dependent terms are assumed negligible. This "semiempirical" $b_{j}\left(E_{j}\right)$ is used with the dipole term to form a projectile-independent Bethe cross section. Then the binary-encounter cross section appropriate for heavy-ion projectiles [Eqs. (16a)-16d)] is added to this "semiempirical" Bethe cross section to account for ejection of fast secondary electrons and to introduce an additional, though weak, $T$ dependence.

Unfortunately, the $T^{1 / 2}$ dependence in the binaryencounter theory, Eq. (16b), is neither $T^{-1}$ expected from the PWBA [cf. Eq. (22)] nor $\ln T / T$ expected from the distortion of plane waves by the target (Kim and Desclaux, 1987). Hence one must be cautious in using Miller's model for incident protons of moderate to low energies ( $<1 \mathrm{MeV})$. Nevertheless, Miller et al. (1987) have successfully applied their model to a number of atoms and molecules for which sufficient experimental SDCS data exist.

\section{B. Dillon-Inokuti model}

Inokuti et al. (1987) have studied analytic properties of $d f / d E$ and of the generalized oscillator strength; they have proposed to fit continuum oscillator strengths as well as the generalized oscillator strength by a power series in the ratio $W / E$ :

$d \sigma / d W=\left(4 \pi a_{0}^{2} R / T\right)[A(W) \ln (T / R)+B(W)]$,

where

$$
\begin{aligned}
A(W) & =\sum_{j}\left[R^{2} /\left(I_{j}+W\right)\right]\left(d f_{j} / d W\right) \\
& =(1-h)^{2} \sum_{n} a_{n} h^{n} \\
B(W) & =(1-h)^{2} \sum_{n} b_{n} h^{n}
\end{aligned}
$$

and

$$
h=W / E .
$$


As is implied by limiting the $T$ dependence in the square brackets of Eq. (27) to the logarithmic term, the model is not meant to represent singly-differential cross sections for low $T$. When there is more than one dominant shell, $h$ in Eqs. (28)-(30) must be defined for each shell, and $A(W)$ and $B(W)$ must be determined for each shell with different power series.

Inokuti et al. (1987) used Eqs. (27)-(30) and fitted proton-impact singly-differential cross sections of $\mathrm{CH}_{4}$, $\mathrm{NH}_{3}$, and $\mathrm{H}_{2} \mathrm{O}$. The power series in $h$ [Eqs. (28) and (29)] reproduces qualitative features in $A(W)$ and $B(W)$ reasonably well when the incident proton energy is high. This method is thus well suited to represent gross features in SDCS's at high $T$. The structure of the power series, however, is too simple to reproduce details in $A$ ( $W$ ), which often serve to distinguish one target molecule from another.

\section{Kim's model}

In order to provide more flexibility to the semiempirical models based on the Bethe theory allowing its application to lower proton energies and to reproduce the shape of the SDCS more faithfully, a new model is presented below, which expands on the models discussed above (Secs. VI.A and VI.B).

For fast protons $\left(T_{0}>0.5 \mathrm{MeV}\right)$, the dipole term, $a_{j}\left(E_{j}\right)$ in Eq. (22), dominates the singly-differential cross section and is largely responsible for individual differences amongst various targets. The continuum oscillator strengths $d f / d E_{j}$ in Eq. (24) can be deduced from experimental or theoretical photoionization cross sections, though theoretical data near ionization threshold are often unreliable. Once the continuum oscillator strengths are known for a wide range of excitation energies $E_{j}$, one can fit formulas similar to Eq. (22) to known SDCS's by assuming simplified forms for functions $b_{j}\left(E_{j}\right), c_{j}\left(E_{j}\right)$, etc. Contributions from different shells must be evaluated separately for multishell atoms and molecules.

The dipole term is sometimes referred to as the softcollision term, since the dipole interaction is dominated by impact parameters much larger than the target size. In contrast, the $b_{j}\left(E_{j}\right)$ term is known as the hardcollision term, representing the contribution of hard collisions with small impact parameters (see Sec. III.G). In reality, $b_{j}\left(E_{j}\right)$ includes contributions from binary collisions described by the Rutherford cross section, Eq. (13), and from another term loosely related to the cutoff momentum transfer beyond which the dipole interaction diminishes rapidly. While this dipole-interaction cutoff term is proportional to $a_{j}\left(E_{j}\right)$, it is also a function of $E_{j}$, which results in an $E_{j}$ dependence that is difficult to predict, although it is independent of the incident energy. We emphasize the fact that the knowledge of the dipole oscillator strengths alone will not be sufficient to determine proton-impact cross sections because of this cutoff momentum transfer, which must be included in $b_{j}\left(E_{j}\right)$.

Kim's model is designed to take advantage of the Platzman plot, i.e., a plot of the ratio $Y$ defined by Eq. (25) versus $R / E$. When we divide the Bethe cross section, Eq. (22), by the Rutherford cross section, Eq. (13), then we have

$$
\begin{aligned}
Y_{\text {Bethe }}= & E(d f / d E) \ln (T / R)+(E / R)^{2} b(E) \\
& +(E / R)^{2} c(E) R / T+\cdots,
\end{aligned}
$$

indicating the dominant role played by the dipole function $E(d f / d E)$ as $T$ increases. In the absence of the dipole interaction, $(E / R)^{2} b(E)$ approaches the occupation number of each shell participating in the ionizing collision as $E$ increases (Kim, 1975a). The model described below uses these properties and introduces additional $T$ dependent terms to provide the flexibility needed to represent cross sections at lower $T$ than other models discussed above. Moreover, the dipole function, $E(d f / d E)$, is fitted by compact analytic expressions that retain all the major features specific to individual atoms and molecules. These analytic expressions provide a far simpler alternative to cumbersome numerical tables of experimental or theoretical differential oscillator strengths.

To illustrate the importance of the dipole interaction in the singly-differential cross section for fast protons, Kim has extracted continuum oscillator strengths $d f / d E_{j}$ from known photoionization cross sections for Ar (Berkowitz, 1979) and $\mathrm{N}_{2}$ (Berkowitz, 1979; Samson et $a l$., 1987). Then $d f / d E_{j}$ was fitted with a linear combination of Gaussian functions

$$
E(d f / d E)=\sum_{i} a_{i} \exp \left\{-\left[\left(R / E-b_{i}\right) / c_{i}\right]^{2}\right\}(R / E)^{d_{i}},
$$

where $a_{i}, b_{i}, c_{i}$, and $d_{i}$ are fitting parameters (see Table II), or with a four-term power series,

$$
E(d f / d E)=\sum_{i} e_{i}(R / E)^{i},
$$

where $e_{i}, i=1-4$ are fitting parameters (see Table II). Using these $d f / d E_{j}$, Kim has fitted experimental SDCS's of $\mathrm{Ar}$ and $\mathrm{N}_{2}$ to a form

$$
Y_{M}=\frac{(E d f / d E) \ln (4 f T / R)\left[1+g R / T+h(R / T)^{2}\right]+Y_{\mathrm{BE}}+N}{1+\exp [k(E-4 T) / R]}
$$

for the valence shells of Ar and $\mathrm{N}_{2}$, where $f, g, h$, and $k$ are fitting parameters and $N$ is the electron occupation number of the shell.

The additional $T$ dependence in the square brackets of the numerator in Eq. (34) allows for a departure from the $\ln T$ dependence predicted by the PWBA, as discussed by Kim and Desclaux (1988). The term $Y_{\mathrm{BE}}$ is introduced to account for the fact that target electrons are bound 
TABLE II. Parameters for fitting photoionization cross sections of Ar and $\mathrm{N}_{2}$ with Gaussian functions [Eq. (32)] or power series [Eq. (33)]. The actual fitting was carried out on $E(d f / d E)$, a dimensionless quantity. Binding energies $I_{j}$ used in converting $W$ into $E_{j}$ are also listed.

\begin{tabular}{|c|c|c|c|c|c|}
\hline Target & $\begin{array}{c}\text { Index } i \text { in } \\
\text { Eq. (31) }\end{array}$ & $a_{i}$ & $b_{i}$ & $c_{i}$ & $d_{i}{ }^{\mathrm{a}}$ \\
\hline \multirow[t]{5}{*}{ Ar, $M$ shell } & 1 & 7 & 0.505 & 0.12 & 0.01 \\
\hline & 2 & 12 & 0.07 & 0.13 & 1 \\
\hline & 3 & 0.3 & 0.6 & 0.05 & 1 \\
\hline & 4 & 11.3 & 0.72 & 0.19 & 3 \\
\hline & 5 & 10 & 0.63 & 0.1 & 3 \\
\hline \multirow{6}{*}{$\mathrm{N}_{2}, L_{A}$ shell $^{\mathrm{b}}$} & 1 & 5.5 & 0.465 & 0.155 & 0 \\
\hline & 2 & 0.8 & 0.59 & 0.035 & 0 \\
\hline & 3 & 3.4 & 0.71 & 0.14 & 0 \\
\hline & 4 & 1.3 & 0.81 & 0.09 & 0 \\
\hline & 5 & 1.6 & 0.26 & 0.08 & 0 \\
\hline & 6 & 10 & 0.11 & 0.1 & 1.1 \\
\hline \multirow[t]{6}{*}{$\mathrm{N}_{2}, L_{B}$ shell ${ }^{\mathrm{b}}$} & 1 & 1.6 & 0.26 & 0.06 & 0 \\
\hline & 2 & 0.7 & 0.36 & 0.055 & 0 \\
\hline & 3 & 0.5 & 0.525 & 0.02 & 2 \\
\hline & 4 & 0.5 & 0.47 & 0.08 & 1 \\
\hline & 5 & 0.6 & 0.12 & 0.08 & 0.1 \\
\hline & 6 & 0.4 & 0.18 & 0.05 & 1 \\
\hline Target & [see Eq. (33)] & $e_{1}$ & $e_{2}$ & $e_{3}$ & $e_{4}$ \\
\hline Ar, $L$ shell & & 48.67 & 745.6 & -141200 & 616300 \\
\hline $\mathrm{N}_{2}, 1 \sigma_{g}+1 \sigma_{u}$ & & 2.99 & 5813 & 30506 & -1987000 \\
\hline Shell & $\mathrm{Ar}, M$ & $\mathrm{Ar}, L$ & $\mathrm{~N}_{2}, L_{A}{ }^{\mathrm{b}}$ & $\mathrm{N}_{2}, L_{B}{ }^{\mathrm{b}}$ & $\mathrm{N}_{2}, K$ \\
\hline $\begin{array}{l}\text { Binding } \\
\text { energy, } I_{j}(\mathrm{eV})\end{array}$ & 15.82 & 249.18 & 15.59 & 28.8 & 410 \\
\hline
\end{tabular}

${ }^{a}$ More careful fitting of the $d_{i}$ parameters is required to reproduce the correct asymptotic (high-energy) behavior of $E(d f / d E)$.

${ }^{\mathrm{b}} L_{A}=3 \sigma_{g}+1 \pi_{u}+2 \sigma_{u} ; L_{B}=2 \sigma_{g}$.

and have intrinsic momentum distributions:

$$
Y_{\mathrm{BE}}=(N U / E)\left\{1-[U /(W+U)]^{p}\right\},
$$

where $U$ is the average kinetic energy of the bound electrons [see Table $I(a)$ ] and $p$ is a fitting parameter. A value of 8 was assigned to the occupation number $N$ in Eq. (34) for the $M$ shell of $\mathrm{Ar}$ and for the $L_{A}$ group $\left(3 \sigma_{g}, 1 \pi_{u}\right.$, and $2 \sigma_{u}$ orbitals) of $\mathrm{N}_{2}$; a value of 2 was assigned for the $L_{B}$ group ( $2 \sigma_{g}$ orbital) of $\mathrm{N}_{2}$. The denominator $W+U$ in Eq. (35) simulates the classical cutoff in energy transfer (see Sec. III.C).

The continuum oscillator strengths for the $M$ shell of Ar and the $L_{A}$ and $L_{B}$ groups of $\mathrm{N}_{2}$ were fitted with five or six Gaussian functions. Contributions from orbitals with similar binding energies are combined, since small differences in these binding energies are insignificant for the modest level of accuracy attainable with this model.

The contributions to $d f / d E$ from the $L$ shell of Ar and the $1 \sigma$ shells of $\mathrm{N}_{2}$ are hydrogenic in shape and were fitted to a power series, Eq. (33). Various binding energies $I_{j}$ used in relating photoelectron energy $W$ to the photon energy, $E_{j}=W+I_{j}$, are also listed in Table II.

The $K$ shell of Ar is ignored in the following discussion because it contributes very little to the ionization cross sections. It will, however, play more significant roles for the stopping power or any other quantity that involves

TABLE III. Parameters for fitting singly-differential cross sections to the Kim model, Eqs. (34)-(36).

\begin{tabular}{|c|c|c|c|c|c|c|c|c|}
\hline Target/shell & $f$ & $g$ & $h$ & $k$ & $p$ & $q$ & $r$ & $N$ \\
\hline Ar, $M$ shell & 0.053 & -5 & 20 & 0.1 & 0.3 & & & 8 \\
\hline Ar, $L$ shell & & & & & & 0.25 & 0.1 & 8 \\
\hline $\mathrm{N}_{2}, L_{A}$ shell $^{\mathrm{a}}$ & 0.3 & -8 & 25 & 0.1 & 0.2 & & & 8 \\
\hline $\mathrm{N}_{2}, L_{B}$ shell $^{\mathrm{a}}$ & 0.3 & -8 & 25 & 0.1 & 0.2 & & & 2 \\
\hline $\mathbf{N}_{2}, 1 \sigma_{g}+1 \sigma_{u}$ & & & & & & 1.0 & 0.1 & 4 \\
\hline
\end{tabular}

${ }^{\mathrm{a}} L_{A}=3 \sigma_{g}+1 \pi_{u}+2 \sigma_{u} ; L_{B}=2 \sigma_{g}$. 
TABLE IV. Experimental absolute proton-impact doubly-differential cross sections. All published absolute cross sections known to the authors are listed here.

Primary energies

Investigators $(\mathrm{keV})$

Hydrogen $(\mathbf{H})$

Park et al., 1977

Helium $(\mathrm{He})$

Blauth, 1957

Rudd and Jorgensen, 1963

Rudd et al., 1966

Park and Schowengerdt, 1969b

Toburen, 1971

Stolterfoht, 1971a

Bordenave-Montesquieu et al., 1973

Manson et al., 1975

Rudd and Madison, 1976

Stolterfoht, 1975

Rødbro and Andersen, 1979

Tokoro and Oda, 1985

Gibson and Reid, 1985, 1986

Schader et al., 1986

Olson et al., 1987

Bernardi et al., 1988

Irby et al., 1988

Bernardi et al., 1989

Cheng et al., 1989a

Bernardi et al., 1989,1990

Gay et al., 1990

Neon $(\mathrm{Ne})$

Blauth, 1957

Crooks and Rudd, 1971

Toburen et al., 1978

Cheng et al., 1989a

Bernardi et al., 1990

Gay et al., 1990

\section{Argon (Ar)}

Blauth, 1957

Crooks and Rudd, 1971

Gabler, 1974

Criswell et al., 1977

Rudd, 1977

Toburen et al., 1978

Toburen et al., 1978

Sataka, Urakawa, and Oda, 1979

Gibson and Reid, 1987a,1987b

\section{Krypton $(\mathrm{Kr})$}

Blauth, 1957

Manson and Toburen, 1977

Cheng et al., 1989a

Xenon (Xe)

Toburen, 1974

Hydrogen $\left(\mathbf{H}_{2}\right)$

Blauth, 1957

Kuyatt and Jorgensen, 1963

Rudd and Jorgensen, 1963

Rudd et al., 1966

Toburen and Wilson, 1972
$25,50,75,145,200$

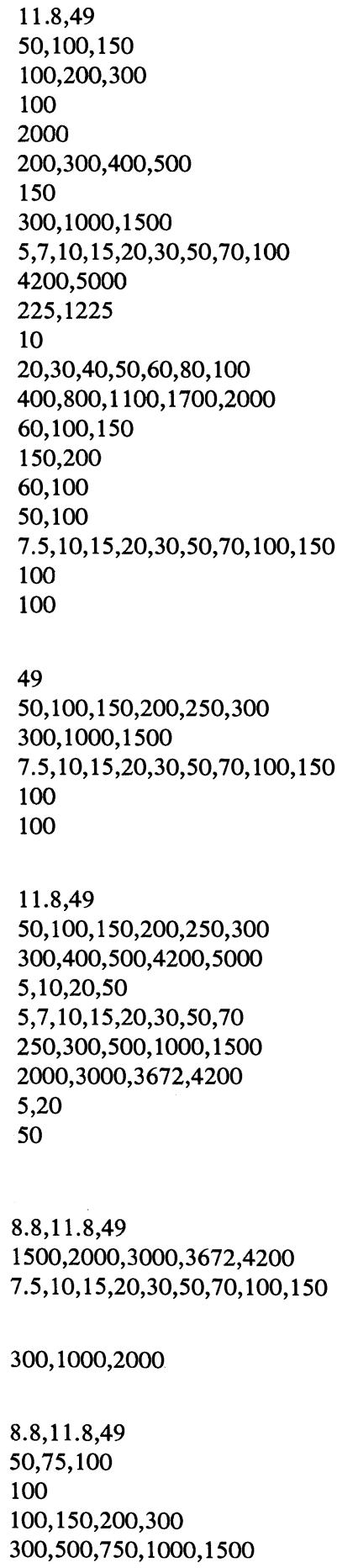


TABLE IV. (Continued).

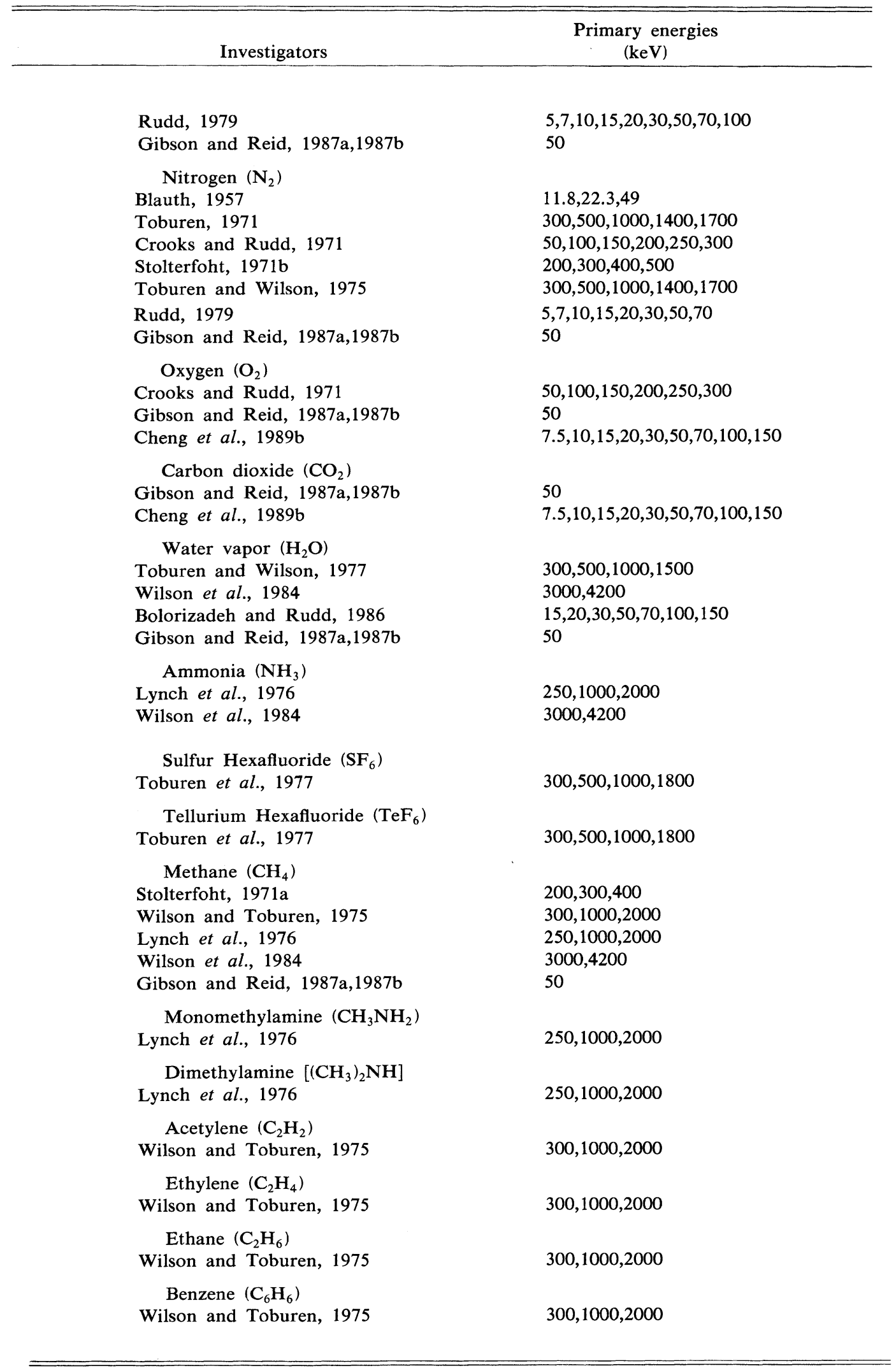


large energy losses of the incident particle. It is difficult to extend the same type of analysis to the $K$ shell of Ar at present for lack of experimental singly-differential cross sections specific to that shell.

Kim used a somewhat simpler form of $Y$ for the $L$ shell of $\mathrm{Ar}$ and the two $1 \sigma$ shells of $\mathrm{N}_{2}$ :

$$
Y_{L}=\frac{(E d f / d E) \ln \left(4 q T E / R^{2}\right)+Y_{\mathrm{BE}}+N}{1+\exp [r(E-4 T) / R]},
$$

with fitting parameters $q$ and $r$. For the $L$ shell of Ar, $N=8$, while $N=4$ for the $1 \sigma$ shell of $N_{2},\left(1 \sigma_{g}+1 \sigma_{u}\right)$. The actual values of the fitting parameters are listed in Table III. The $Y$ function for the entire target is obtained by summing $Y_{M}$ and $Y_{L}$.

Experimental singly-differential cross-section data for Ar and $\mathrm{N}_{2}$ (see Table IV) were used to determine the values of these fitting parameters so that the major features in the profiles of the Platzman plots are faithfully represented and the integrated cross sections (total ionization cross sections) are consistent with those recommended in Rudd et al. (1985). The many fitting parameters are independent of the ejected-electron energy as well as of the incident-proton energy for the entire range of $W$ and for $T_{0}>200 \mathrm{keV}$. Since this model is consistent with the Bethe theory and uses realistic oscillator strengths, it is also expected to be reliable for very fast protons $\left(T_{0} \gtrsim 10 \mathrm{MeV}\right)$, though relativistic effects are not included in this model and will eventually dominate at extremely high incident energies.

For protons of relativistic velocity $v$, or $\beta=v / c>0.1$ where $c$ is the speed of light, relativistic expressions for kinematic variables such as energy and momentum must be used and an additional interaction between the proton and bound electrons must be included. The net result is that Eq. (22) is rewritten as

$$
d \sigma / d W=\left(4 \pi a_{0}^{2} \alpha^{2} / \beta^{2}\right) \sum_{j}\left(a_{j}\left(E_{j}\right)\left\{\ln \left[\beta^{2} /\left(1-\beta^{2}\right)\right]-\beta^{2}\right\}+b_{j}^{\prime}\left(E_{j}\right)+\cdots\right),
$$

with

$$
b_{j}^{\prime}\left(E_{j}\right)=b_{j}\left(E_{j}\right)-2 a_{j}\left(E_{j}\right) \ln \alpha,
$$

where $\alpha \cong \frac{1}{137}$ is the fine-structure constant.

The optical functions $E(d f / d E)$ of $\mathrm{Ar}$ and $\mathrm{N}_{2}$ obtained from this model are presented in Figs. 6 and 7, respectively. Fitted singly-differential cross sections are presented in Platzman plots in Figs. 8-11 and compared with available experimental data.

Peaks in experimental data for Ar that are associated with Auger electrons released following the ionization of $L$-shell electrons (near $R / E=0.07$ ) are not included in Kim's model; they appear as sharp peaks in the Platzman

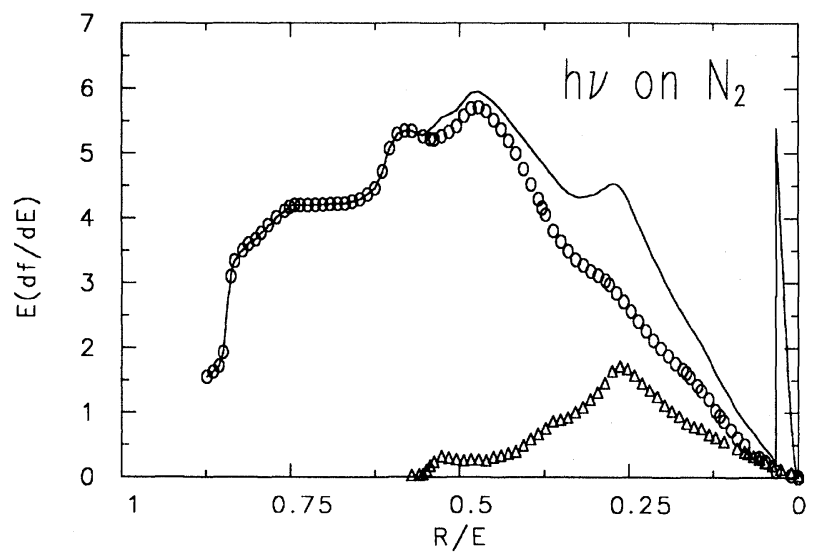

FIG. 7. The dipole function $E(d f / d E)$ of $\mathrm{N}_{2}$ as a function of the inverse of the photon energy in rydbergs. The solid curve is a fit to the smoothed experimental data of Samson et al. (1987) and those compiled by Berkowitz (1979): $\bigcirc$, direct ionization resulting in $\mathrm{N}_{2}^{+} ; \triangle$, dissociative ionization resulting in $\mathrm{N}^{+}$and N. plot and add little to the total ionization cross section (Figs. 8 and 9). Experimental and model values are in excellent agreement for Ar except for slow ejected electrons $(W<10 \mathrm{eV})$ at $T_{0}=300 \mathrm{keV}$.

Similar comparisons for $\mathrm{N}_{2}$ data (Figs. 10 and 11) do not show agreement equal to that observed for Ar. It is likely that the quality of available experimental data for $\mathrm{N}_{2}$ with fast protons $\left(T_{0}>0.5 \mathrm{MeV}\right)$ is poorer than that

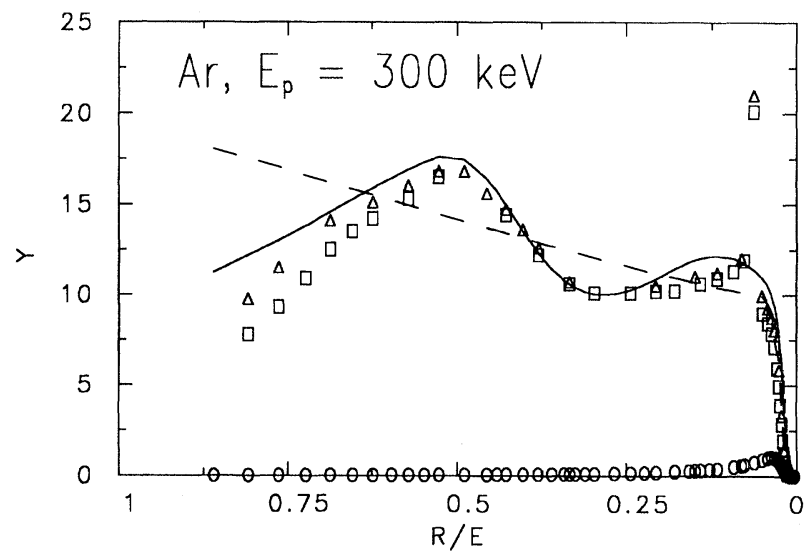

FIG. 8. Platzman plot of proton-impact $\left(T_{0}=300 \mathrm{keV}\right)$ singlydifferential cross section of Ar. The solid curve is the recommended SDCS based on Kim's model: $O, L$-shell contribution; $\square$, experimental data of Crooks and Rudd (1971); $\triangle$, experimental data of Toburen, Manson, and Kim (1978). The sharp peak near $R / E=0.07$ consists of several peaks due to the LMM Auger transitions. The peak expected at $R / E \approx 0.077$ from charge transfer to the continuum is masked by the Auger peaks, but the former, although it is expected to be broader, is not noticeable. The dashed curve is the SDCS based on Rudd's model, discussed in Sec. VI.D. 


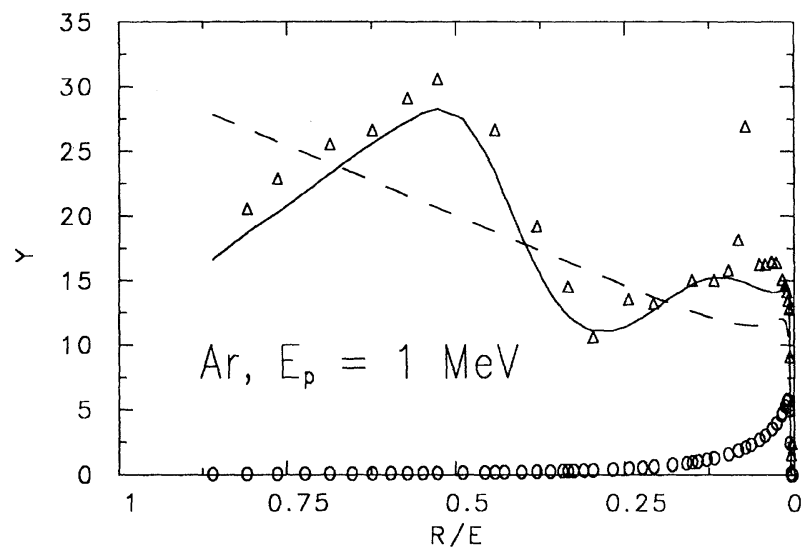

FIG. 9. Platzman plot of proton-impact $\left(T_{0}=1 \mathrm{MeV}\right)$ SDCS of Ar. See Fig. 8 for the legend.

for Ar (Toburen, 1990). As a result, fitted formulas for $\mathrm{N}_{2}$ have greater uncertainty, but the model should adequately represent the systematics of the singly-differential cross sections for fast protons. Auger electrons emitted following $K$-shell ionization appear as sharp peaks near $R / E=0.03$, again contributing little to the total ionization cross section.

For moderate to slow incident protons $\left(T_{0}<500 \mathrm{keV}\right)$, charge transfer to the continuum (see Sec. III.D) becomes apparent. This contribution is localized near electron speeds matching that of the incident proton (e.g., $W \approx 160 \mathrm{eV}$ for $T_{0}=300 \mathrm{keV}$ ), but it is difficult to describe theoretically and hence was omitted in Kim's model. A continuum-charge-transfer "hump" can be seen in the vicinity of $R / E=0.15$ in Fig. 10 for $\mathrm{N}_{2}$. A similar

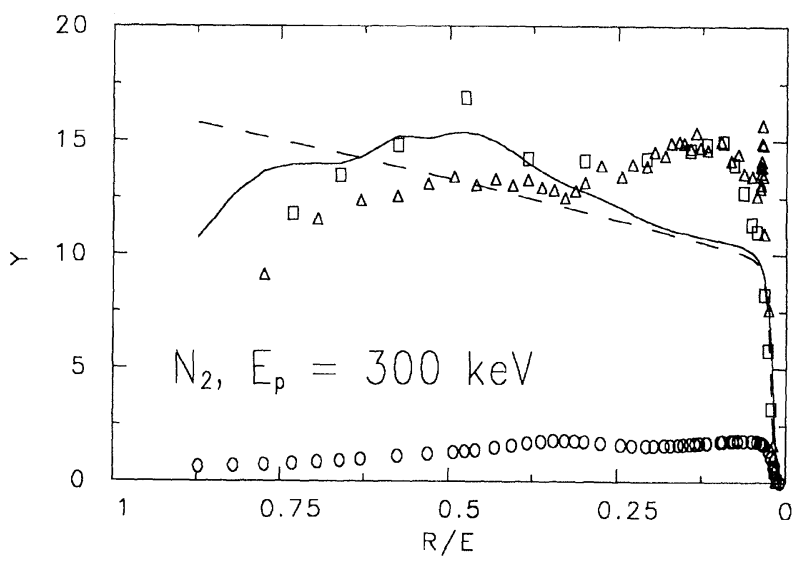

FIG. 10. Platzman plot of proton-impact $\left(T_{0}=300 \mathrm{keV}\right)$ singly-differential cross section of $\mathrm{N}_{2}$. The solid curve is the recommended SDCS based on Kim's model: $O$, contribution from the $2 \sigma_{g}$ subshell; $\triangle$, experimental data of Toburen (1971), which was later improved by Toburen and Wilson (1975); $\square$, data of Crooks and Rudd (1971). The broad peak at $R / E \approx 0.1$ arises from charge transfer to the continuum, and the sharp peak at $R / E \approx 0.03$ is due to KLL Auger electrons. The broken curve is the SDCS based on Rudd's model.

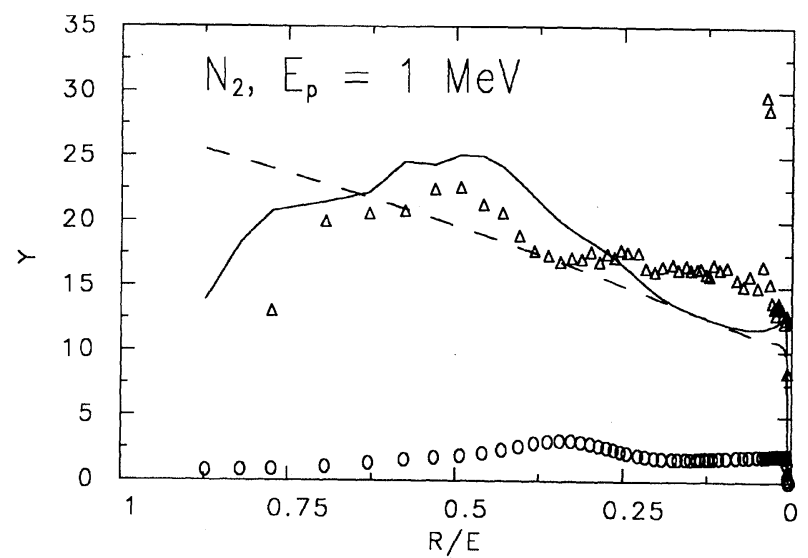

FIG. 11. Platzman plot of proton-impact $\left(T_{0}=1 \mathrm{MeV}\right) \mathrm{SDCS}$ of $\mathrm{N}_{2}$. See Fig. 10 for the legend.

hump in Ar is not visible in Fig. 8; it may have been masked by the Auger peaks in the vicinity.

At much lower incident-proton energies $\left(T_{0}<300\right.$ $\mathrm{keV}$ ), the dipole interaction does not dominate and the Bethe theory, on which Kim's model is based, becomes inadequate. There is no definitive theory for this region. The fitting formulas shown above should not be used for $T_{0}<200 \mathrm{keV}$.

Kim's model can be extended to other targets for which experimental data are available to determine the fitting parameters. Helium, neon, krypton, xenon, molecular hydrogen, water, ammonia, and methane are candidates for detailed future studies. Photoionization cross sections for these atoms and molecules are known with better accuracy than the corresponding proton-impact singly-differential cross sections. Kim's model should also be applicable to electron-impact ionization of atoms and molecules with minor adjustments of the fitting parameters.

Kim's model and that of Miller et al. (Sec. VI.A) have equivalent major features, but Kim's model includes additional $T$-dependent terms that extend its applicability toward lower proton energies. However, these additional $T$-dependent terms specifically depend on the type of projectile, thus making it necessary to introduce different fitting parameters for electron-impact and proton-impact ionization cross sections.

\section{Rudd's model}

This model (Rudd 1987, 1988) is designed to provide an analytic representation of the differential cross sections over a wide range of primary and secondary energies. It requires experimental data to determine its parameters, but successfully bridges large gaps in intermediate energy ranges. An important advantage of this model is that it has no restrictions on primary and secondary energies and therefore is useful even at impact velocities well below the target's orbital velocity.

This model assumes that the cross section for ejection 
of an electron depends only on the secondary energy, the binding energy of the electron, and the projectile velocity. This leads to the relation

$$
\frac{d \sigma}{d W}=\sum_{i} N_{i} f\left(W, T, I_{i}\right)
$$

where $N_{i}$ is the number of electrons in a shell with binding energy $I_{i}, W$ is the energy of the ejected electron, and $T=(m / M) T_{0}$, where $T_{0}$ is the proton energy. The sum is taken over all shells of the target. It further assumes that the ratios of the primary and secondary energies to the binding energy are the important quantities. This assumption and other considerations lead to the relation

$$
\frac{d \sigma}{d W}=\sum_{i}\left(N_{i} / I_{i}^{2}\right) f\left(w_{i}, v_{i}\right)
$$

where

$$
w_{i}=W / I_{i} \text { and } v_{i}=\left(T / I_{i}\right)^{1 / 2} .
$$

Using this notation, we may write a general crosssection equation for a single shell:

$$
\frac{d \sigma}{d W}=(S / I)\left(F_{1}+F_{2} w\right) /(1+w)^{3},
$$

where $S=4 \pi a_{0}^{2} N(R / I)^{2}$. In Eq. (40), the units of $d \sigma / d W$ are determined solely by the choice of units for $S$ and $I$, since the remaining terms are dimensionless.

The results of Rutherford (1911) and Thomson (1912) on the scattering of charged particles from atoms may be used to obtain an equation for the cross section assuming that each target electron is initially at rest (see Sec. III.E). This relation is given by Eq. (40) with $F_{1}=F_{2}=1 / v^{2}$, which then becomes Eq. (13). Williams (1927) took into account the initial motion of the target electron and obtained a result that may also be put into the form of Eq. (40) but with $F_{1}=7 / 3 v^{2}$ and $F_{2}=1 / v^{2}$. This choice leads to Eq. (16) with $U=I$, which holds exactly for the hydrogen atom and one-electron ions and as an approximation for other targets.

In the Rudd model, $F_{1}$ and $F_{2}$ are taken to be adjustable fitting parameters that are functions of $v$ determined from experimental data. In addition, it is required that $F_{1} \rightarrow\left(1 / v^{2}\right) \log \left(v^{2}\right)$ and $F_{2} \rightarrow 1 / v^{2}$ for $v \gg 1$, thus reproducing the well-verified asymptotic energy dependence of the Bethe theory.

Equation (40), however, does not give the proper dependence on secondary-electron energy above the kinematic cutoff. This is corrected in the model by the addition of a factor derived from the molecular promotion model (Rudd, 1979, 1988). The final equation is then

$$
\sigma(w)=\frac{(S / I)\left(F_{1}+F_{2} w\right)(1+w)^{-3}}{1+\exp \left[\alpha\left(w-w_{c}\right) / v\right]},
$$

where $\alpha$ is a dimensionless parameter near unity related to the size of the target and $w_{c}$, the energy at the cutoff, is

$$
w_{c}=4 v^{2}-2 v-R / 4 I .
$$

The first term on the right-hand side represents the freeelectron limit, $4 T$, discussed in Appendix $\mathrm{A}$, the second term represents the correction due to electron binding (Rudd, 1988), and the third term gives the correct dependence for $v \ll 1$.

The quantities $F_{1}, F_{2}$, and $\alpha$ constitute the three adjustable parameters in the model for each secondaryelectron spectrum at a given primary energy. The quantity $\alpha$ resulting from the fitting turns out to be essentially independent of primary energy and is taken to be a constant for each target. The other two quantities are functions of the primary energy that may be fitted to the experimental data, subject to the asymptotic energy dependence given above, by the equations

$$
F_{1}(v)=L_{1}+H_{1}
$$

and

\begin{tabular}{|c|c|c|c|c|c|c|c|c|c|c|c|}
\hline & $\mathrm{He}$ & $\mathrm{Ne}$ & Ar & $\mathrm{Kr}$ & $\mathbf{H}_{2}$ & $\mathbf{N}_{2}$ & $\mathrm{O}_{2}$ & $\mathrm{H}_{2} \mathrm{O}$ & $\mathrm{CO}_{2}$ & $\mathrm{CH}_{4}$ & $\begin{array}{l}\text { Inner } \\
\text { shells }\end{array}$ \\
\hline$A_{1}$ & 1.02 & 0.58 & 1.20 & 1.46 & 0.96 & 1.05 & 1.02 & 0.97 & 1.09 & 1.15 & 1.25 \\
\hline$B_{1}$ & 2.4 & 65 & 8.0 & 5.7 & 2.6 & 12.0 & 50 & 82 & 25 & 14 & 0.50 \\
\hline$C_{1}$ & 0.70 & 0.23 & 0.86 & 0.65 & 0.38 & 0.74 & 0.40 & 0.40 & 0.75 & 0.35 & 1.00 \\
\hline$D_{1}$ & 1.15 & 0.55 & 0 & -0.55 & 0.23 & -0.39 & 0.12 & -0.30 & 0.75 & 0.50 & 1.00 \\
\hline$E_{1}$ & 0.70 & 0.16 & 0.80 & 1.00 & 2.2 & 0.80 & 0.30 & 0.38 & 0.65 & 3.0 & 3.0 \\
\hline$A_{2}$ & 0.84 & 1.40 & 0.90 & 1.30 & 1.04 & 0.95 & 1.00 & 1.04 & 0.78 & 0.60 & 1.10 \\
\hline$B_{2}$ & 6.0 & 0 & 2.7 & 22 & 5.9 & 1.20 & 5.0 & 17.3 & 3.0 & 3.8 & 1.30 \\
\hline$C_{2}$ & 0.70 & 0.72 & 0.75 & 0.95 & 1.15 & 1.00 & 0.55 & 0.76 & 0.70 & 1.20 & 1.00 \\
\hline$D_{2}$ & 0.50 & 1.35 & 0.80 & -1.00 & 0.20 & 1.30 & 0 & 0.04 & 0.85 & 0.45 & 0 \\
\hline$\alpha$ & 0.86 & 0.57 & 0.71 & 0.78 & 0.87 & 0.70 & 0.59 & 0.64 & 0.53 & 0.61 & 0.66 \\
\hline
\end{tabular}

$$
F_{2}(v)=L_{2} H_{2} /\left(L_{2}+H_{2}\right),
$$

with

$$
H_{1}=A_{1} \ln \left(1+v^{2}\right) /\left(v^{2}+B_{1} / v^{2}\right),
$$

TABLE V. Parameters for fitting singly-differential cross sections to the Rudd model, Eqs. (41)-(48). 


$$
\begin{aligned}
& L_{1}=C_{1} v^{D_{1}} /\left[1+E_{1} v^{\left(D_{1}+4\right)}\right], \\
& H_{2}=A_{2} / v^{2}+B_{2} / v^{4},
\end{aligned}
$$

TABLE VI. Summary of experimental apparatuses by laboratory. That of Rødbro and Andersen (1979) is not included in this listing. Substantial changes in apparatus are indicated by different numbers, as are separate apparatuses. The "overall estimated error" category contains the most conservative errors quoted by the authors in a given series of references associated with the specific apparatus. Generally, the authors referenced here have made the common mistake of combining systematic errors quadratically, instead of linearly. Where possible, i.e., when individual systematic errors are specified, we have quoted a more conservative linear error. A comprehensive reference list of all known absolute

\begin{tabular}{|c|c|c|c|c|}
\hline Laboratory & Apparatus & References & $\begin{array}{c}\text { Proton-energy } \\
\text { range }(\mathrm{keV}) / \text { targets }\end{array}$ & Analyzer \\
\hline $\begin{array}{l}\text { University of } \\
\text { Nebraska, } \\
\text { Lincoln, NE }\end{array}$ & 1 & Kuyatt and Jorgensen (1963) & $50-100 ; \mathrm{H}_{2}$ & $127^{\circ}$ \\
\hline \multirow{4}{*}{$\begin{array}{l}\text { [apparatus } 3 \\
\text { constructed at } \\
\text { Concordia Col- } \\
\text { lege, Moorhead, } \\
\text { MN, where the } \\
\text { work of Rudd } \\
\text { et al. (1966) was } \\
\text { done] }\end{array}$} & 2 & $\begin{array}{l}\text { Rudd and Jorgensen (1963) } \\
\text { Rudd et al. (1976) }\end{array}$ & $50-150 ; \mathrm{H}_{2} \mathrm{He}$ & $127^{\circ}$ \\
\hline & 3 & $\begin{array}{l}\text { Rudd et al. (1966) } \\
\text { Rudd et al. (1976) }\end{array}$ & $\begin{array}{l}100-300 \\
\mathrm{H}_{2}, \mathrm{He}\end{array}$ & $\begin{array}{l}\text { Parallel- } \\
\text { plate }\end{array}$ \\
\hline & 4 & $\begin{array}{l}\text { Crooks and Rudd (1971) } \\
\text { Rudd et al. (1979) }\end{array}$ & $\begin{array}{l}50-300 ; \\
\mathrm{N}_{2}, \mathrm{O}_{2}, \mathrm{Ne}, \mathrm{Ar}\end{array}$ & $\begin{array}{l}127^{\circ} \\
127^{\circ}\end{array}$ \\
\hline & 5 & $\begin{array}{l}\text { Rudd and Madison (1976) } \\
\text { Rudd et al. (1976,1979) } \\
\text { Rudd (1977) } \\
\text { Rudd (1979) } \\
\text { Bolorizadeh and Rudd (1986) }\end{array}$ & $\begin{array}{l}5-150 ; \mathrm{He} \\
\mathrm{N}_{2}, \mathrm{H}_{2}, \mathrm{Ar}, \\
\mathrm{H}_{2} \mathrm{O}, \mathrm{Ne}, \\
\mathrm{Kr}, \mathrm{O}_{2}, \mathrm{CO}_{2} \\
\mathrm{Xe}\end{array}$ & $\begin{array}{c}127^{\circ} \% \\
\text { Parallel- } \\
\text { plate }\end{array}$ \\
\hline
\end{tabular}
doubly-differential cross sections is contained in Table IV.

Cheng et al. (1989a,1989b)

\begin{tabular}{|c|c|c|c|c|c|}
\hline Laboratory & Apparatus & Target type & $\begin{array}{l}\text { Detector } \\
\text { type }\end{array}$ & $\begin{array}{l}\text { Quoted } \\
\text { magnetic } \\
\text { fields }(T)\end{array}$ & $\begin{array}{l}\text { Analyzer } \\
\text { pre- or post- } \\
\text { accelerator }\end{array}$ \\
\hline \multirow[t]{5}{*}{$\begin{array}{l}\text { University of } \\
\text { Nebraska, } \\
\text { Lincoln, NE }\end{array}$} & 1 & $\begin{array}{l}\text { Gas fills } \\
\text { vacuum } \\
\text { chamber } \\
\text { (static) }\end{array}$ & $\begin{array}{l}\text { Electron } \\
\text { multiplier } \\
\text { (From PM } \\
\quad \text { tube) }\end{array}$ & $\begin{array}{l}10^{-7} ; \\
\text { Helmholtz } \\
\text { coils }\end{array}$ & Both \\
\hline & 2 & Static & $\begin{array}{l}\text { Electron } \\
\text { multiplier } \\
\text { (From PM } \\
\quad \text { tube) }\end{array}$ & $\begin{array}{l}5 \times 10^{-7} \\
\text { Helmholtz } \\
\text { coils }\end{array}$ & Both \\
\hline & 3 & Static & $\begin{array}{l}\text { Electron } \\
\text { multiplier }\end{array}$ & $\begin{array}{l}5 \times 10^{-7} \\
\text { Helmholtz } \\
\text { coils }\end{array}$ & Both \\
\hline & 4 & Static & $\begin{array}{l}\text { Electron } \\
\text { multiplier }\end{array}$ & $\begin{array}{l}<5 \times 10^{-7} \\
\text { Helmholtz } \\
\text { coils }\end{array}$ & Both \\
\hline & 5 & Static & $\begin{array}{l}\text { Electron } \\
\text { multiplier }\end{array}$ & $\begin{array}{l}<5 \times 10^{-7} \\
\text { Helmholtz } \\
\text { coils }\end{array}$ & Post only \\
\hline
\end{tabular}

Gay et al. (1990) 
The ten basic parameters $A_{1}, \ldots, E_{1}, A_{2}, \ldots, D_{2}$ and $\alpha$ for a given target suffice to specify the cross section at any combination of primary and secondary energies.

A different but universal set of parameters was found to be more appropriate for inner-shell orbitals. Inner shells are defined for this purpose as those whose binding energy $I$ exceeds twice the binding energy of the least tightly bound orbital. These parameters are listed in Table $\mathrm{V}$ for ten targets. The quantities $A_{1}$ and $A_{2}$, which are approximately unity, are related to the first Bethe coefficient, $a(E)$ of Eqs. (20) and (21), and thus to the integrated optical oscillator strength obtainable from photoionization data. The relations are

$$
A_{1}=\frac{2 a(E)}{\sum_{i}\left(N_{i} R / I_{i}\right)}
$$

and

$$
A_{2}=2-A_{1} .
$$

\section{EXPERIMENTAL METHODS}

Two general experimental techniques have been used to determine the singly-differential cross sections reported in this article. The first involves measurement of the

TABLE VI. (Continued).

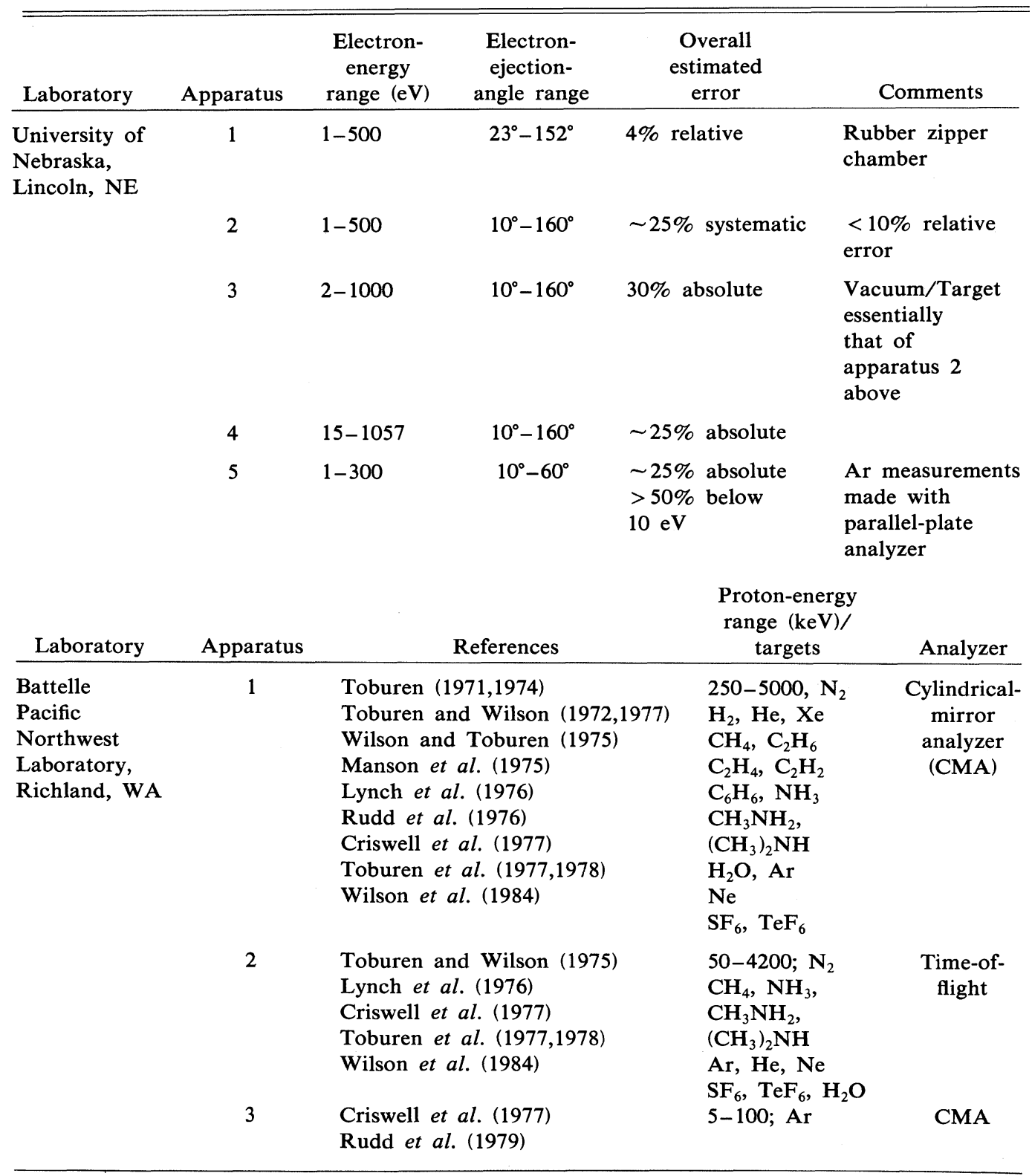


energy and angle of ejection for ionized electrons, i.e., doubly-differential cross sections. These can be integrated to yield cross sections differential either in energy or angle. The second method is ion-energy-loss spectroscopy, in which the proton energy is analyzed following the collision. Those protons which lose energy greater than that corresponding to the ionization limit of the target contribute to the ionization cross section, except when the energy is lost to dissociation or photon production. Measurements made to date using this technique yield directly cross sections that are singly differential in energy. Almost all of the data reported here have been taken using the first method.

\section{A. Analysis of ejected electrons}

A schematic diagram of the generic apparatus employed in the electron-analysis method is shown in Fig. 12. (Table VI lists specific characteristics of most of the apparatuses used in the work considered in this review.) Proton beams with typical ion-source energy spreads $(<100 \mathrm{eV})$ are magnetically analyzed and directed toward the scattering apparatus. At the entrance of the scattering chamber they are collimated and pass through the target region. Collimation is necessary to properly define the scattering geometry, but it can cause secondary and tertiary problems. Secondary electrons produced at

TABLE VI. (Continued).

\begin{tabular}{|c|c|c|c|c|c|}
\hline Laboratory & Apparatus & Target type & $\begin{array}{c}\text { Detector } \\
\text { type }\end{array}$ & $\begin{array}{l}\text { Quoted } \\
\text { magnetic } \\
\text { fields }(T)\end{array}$ & $\begin{array}{c}\text { Analyzer } \\
\text { pre- or post- } \\
\text { acceleration }\end{array}$ \\
\hline \multirow[t]{4}{*}{$\begin{array}{l}\text { Battelle } \\
\text { Pacific } \\
\text { Northwest } \\
\text { Laboratory, } \\
\text { Richland, WA }\end{array}$} & 1 & $\begin{array}{l}\text { Static gas } \\
\text { cell }\end{array}$ & $\begin{array}{l}\text { Channel-electron } \\
\text { multiplier } \\
\text { (CEM) }\end{array}$ & $\begin{array}{l}\sim 2 \times 10^{-7} \\
\text { Helmholtz } \\
\text { coils } \\
{\left[<2 \times 10^{-6}\right.} \\
\text { in Toburen and } \\
\text { Wilson }(1977)]\end{array}$ & None \\
\hline & 2 & $\begin{array}{l}\text { Gas beam from } \\
\text { multicapillary } \\
\text { array }\end{array}$ & CEM & $\begin{array}{l}<2 \times 10^{-7} \\
\text { magnetic } \\
\text { shield plus } \\
\text { Helmholtz } \\
\text { coils }\end{array}$ & Post \\
\hline & 3 & $\begin{array}{l}\text { Gas beam from } \\
\text { multicapillary } \\
\text { array }\end{array}$ & CEM & $\begin{array}{l}<5 \times 10^{-7} \\
\text { magnetic } \\
\text { shield plus } \\
\text { Helmholtz } \\
\text { coils }\end{array}$ & Both \\
\hline & Apparatus & $\begin{array}{l}\text { Electron- } \\
\text { energy } \\
\text { range }(\mathrm{eV})\end{array}$ & $\begin{array}{c}\text { Electron- } \\
\text { ejection- } \\
\text { angle range }\end{array}$ & $\begin{array}{l}\text { Overall } \\
\text { estimated } \\
\text { error }\end{array}$ & Comments \\
\hline \multirow[t]{3}{*}{$\begin{array}{l}\text { Battelle } \\
\text { Pacific } \\
\text { Northwest } \\
\text { Laboratory, } \\
\text { Richland, WA }\end{array}$} & 1 & $0-4000$ & $15^{\circ}-130^{\circ}$ & $\begin{array}{l}\sim 25 \% \text { absolute } \\
>50 \% \\
\text { (statistical) } \\
\text { at high } \\
\text { ejection } \\
\text { energies }\end{array}$ & \\
\hline & 2 & $0-500$ & $50^{\circ}-130^{\circ}$ & $\begin{array}{l}\sim 10 \% \text { relative } \\
>20 \mathrm{eV} \\
>50 \% \\
\text { relative }<1 \mathrm{eV} \\
\text { (statistical) }\end{array}$ & $\begin{array}{l}\text { Nominally } \\
50 \mathrm{~V} \\
\text { acceleration } \\
\text { into CEM }\end{array}$ \\
\hline & 3 & $0-225$ & $30^{\circ}-145^{\circ}$ & $\begin{array}{l}\sim 10 \% \text { relative } \\
\sim 25 \% \text { absolute } \\
\text { (normalized } \\
\text { with } \\
\text { apparatus } 1 \text { ) }\end{array}$ & \\
\hline
\end{tabular}


the edges of the collimating apertures must be prevented from entering the scattering volume. This is usually accomplished by a suppressor biased at a negative voltage $(-100 \mathrm{~V}$ is generally sufficient) after the last collimator. Alternatively, the last collimator may be biased positively. Electric fields from the biased aperture or collimator must now be kept from the scattering volume so that ionized-electron trajectories will not be disturbed. This is accomplished with a grounded, conducting shield, which also helps stop slit-scattered ions from reaching the collision volume.

Three basic scattering volume/target configurations have been used in this type of experiment (see also Table VI). The first, used by Rudd and co-workers (e.g., Rudd and Jorgensen, 1963; Rudd et al., 1966; Crooks and Rudd, 1971; Rudd and Madison, 1976; Criswell et al., 1977; Rudd, 1979; Bolorizadeh and Rudd, 1986; Cheng et al., 1989a, 1989b), involves filling a relatively large volume with a uniform pressure of target gas. The gas is contained in a cylindrical chamber whose only openings are an entrance port for the proton beam and an exit aperture placed at one of several angles for the ejected electrons (Fig. 13). A concentric, cylindrical outer chamber is evacuated by a diffusion pump. Electrons to

TABLE VI. (Continued).

\begin{tabular}{|c|c|c|c|c|c|c|c|}
\hline Laboratory & Apparatus & \multicolumn{2}{|c|}{ References } & \multicolumn{3}{|c|}{$\begin{array}{l}\text { Proton-energy } \\
\text { range }(\mathrm{keV}) \\
\text { targets }\end{array}$} & Analyzer \\
\hline $\begin{array}{l}\text { Hahn-Meitner } \\
\text { Institute, } \\
\text { Berlin, Germany }\end{array}$ & 1 & \multicolumn{3}{|c|}{$\begin{array}{l}\text { Stolterfoht (1971a,1971b,1975) } \\
\text { Stolterfoht et al. (1976) } \\
\text { Gabler (1974) }\end{array}$} & \multicolumn{2}{|c|}{$\begin{array}{l}200-5000 \\
\mathrm{He}, \mathrm{CH}_{4}, \mathrm{~N}_{2} \\
\mathrm{Ar}\end{array}$} & $\begin{array}{l}\text { Parallel- } \\
\text { plate }\end{array}$ \\
\hline $\begin{array}{l}\text { Lucas Heights } \\
\text { Research Laboratory, } \\
\text { Sutherland, Australia }\end{array}$ & 1 & \multicolumn{3}{|c|}{$\begin{array}{l}\text { Gibson and Reid }(1984,1985 \text {, } \\
1986,1987 a, 1987 b)\end{array}$} & \multicolumn{2}{|c|}{$\begin{array}{l}20-100 \\
\mathrm{H}_{2}, \mathrm{He}, \\
\mathrm{O}_{2}, \mathrm{~N}_{2}, \mathrm{CO}_{2} \\
\mathrm{CH}_{4}, \mathrm{H}_{2} \mathrm{O}, \mathrm{Ar}\end{array}$} & $\begin{array}{c}\text { Fountain } \\
\text { parallel- } \\
\text { plate }\end{array}$ \\
\hline $\begin{array}{l}\text { Université } \\
\text { Paul Sabatier, } \\
\text { Toulouse, France }\end{array}$ & 1 & \multicolumn{3}{|c|}{$\begin{array}{l}\text { Bordenave-Montesquieu (1973) } \\
\text { Bordenave-Montesquieu } \\
\text { et al. (1973,1982) } \\
\text { Benoit-Cattin et al. (1973) }\end{array}$} & \multicolumn{2}{|c|}{$14-150 ; \mathrm{He}$} & $127^{\circ}$ \\
\hline \multirow[t]{2}{*}{$\begin{array}{l}\text { Tokyo Institute } \\
\text { of Technology, } \\
\text { Tokyo, Japan }\end{array}$} & 1 & \multicolumn{2}{|c|}{$\begin{array}{l}\text { Sataka et al. (1979) } \\
\text { Tokoro and Oda (1985) }\end{array}$} & \multicolumn{3}{|c|}{$5-30 ; \mathrm{He}, \mathrm{Ar}$} & $\begin{array}{l}\text { Parallel- } \\
\text { plate }\end{array}$ \\
\hline & Apparatus & Target type & $\begin{array}{c}\text { Detector } \\
\text { type }\end{array}$ & \multicolumn{2}{|c|}{$\begin{array}{l}\text { Quoted } \\
\text { magnetic } \\
\text { fields }(\mathrm{T})\end{array}$} & \multicolumn{2}{|c|}{$\begin{array}{l}\text { Analyzer } \\
\text { pre- or post- } \\
\text { acceleration }\end{array}$} \\
\hline $\begin{array}{l}\text { Hahn-Meitner } \\
\text { Institute, } \\
\text { Berlin, Germany }\end{array}$ & 1 & $\begin{array}{l}\text { Static (for } \\
\text { absolute } \\
\text { measurements) } \\
\text { and gas beam } \\
\text { from single } \\
\text { capillary }\end{array}$ & $\begin{array}{l}\text { Electron } \\
\text { multiplier }\end{array}$ & $\begin{array}{l}<3 \times 1 \\
\text { magne } \\
\text { shield }\end{array}$ & & $\begin{array}{l}\text { None } \\
\text { (Preac } \\
\text { used } \\
\text { to } 19\end{array}$ & $\begin{array}{l}\text { celeration } \\
\text { rior } \\
\text { 5) }\end{array}$ \\
\hline $\begin{array}{l}\text { Lucas Heights } \\
\text { Research Laboratory, } \\
\text { Sutherland, Australia }\end{array}$ & 1 & $\begin{array}{l}\text { Gas beam } \\
\text { from } \\
\text { multicapillary } \\
\text { array }\end{array}$ & $\begin{array}{l}\text { CEMs and } \\
\text { an electron } \\
\text { multiplier } \\
\text { at } 0^{\circ}\end{array}$ & $\begin{array}{l}<10^{-} \\
\text {Helmh } \\
\text { coils }\end{array}$ & oltz & None & \\
\hline $\begin{array}{l}\text { Université } \\
\text { Paul Sabatier, } \\
\text { Toulouse, France }\end{array}$ & 1 & Static & CEM & $\begin{array}{l}5 \times 10^{-} \\
\text {Helmh } \\
\text { coils }\end{array}$ & $\begin{array}{l}7 \\
\text { oltz }\end{array}$ & Post & \\
\hline $\begin{array}{l}\text { Tokyo Institute } \\
\text { of Technology, } \\
\text { Tokyo, Japan }\end{array}$ & 1 & $\begin{array}{l}\text { Gas beam } \\
\text { from } \\
\text { multicapillary } \\
\text { array }\end{array}$ & CEM & $\begin{array}{l}<5 \times 1 \\
\text { Magne } \\
\text { shield } \\
\text { (after } \\
\text { and H } \\
\text { coils }\end{array}$ & $\begin{array}{l}0^{-7} \\
\text { tic } \\
980) \\
\text { lmholtz }\end{array}$ & Not $s$ & pecified \\
\hline
\end{tabular}


be analyzed pass through a radial tube in the outer chamber before entering the analyzer. This tube contains several ports for differential pumping. The observation angles in this design are fixed; to change angles the entire apparatus is raised to atmospheric pressure and the radial tube and analyzer chamber are demounted and reinserted in the next port. A modification of this arrangement has been used by Stolterfoht (1971a, 1971b; Fig. 14), Gabler (1974), and Bordenave-Montesquieu and coworkers (1973, 1982; Benoit-Cattin et al., 1973), in which the entire vacuum vessel containing the analyzer is filled with target gas. In the latter case, the analyzer is contained in a box rotatable about the collision center and pumped separately through a flexible tube. One advantage of this design is the ease with which observation angles can be changed.

An early apparatus of this type was described by Kuyatt and Jorgensen (1963) and Cook (1955). In their design, the ejection angle is varied by rotating the scattering chamber and analyzer about the target center, with the incident ion beam fixed in space. A sliding seal over the proton entrance port is made with two greaseimpregnated rubber diaphragms. While this design also has the advantage of not requiring a break in the vacuum to change ejection angles, the diaphragms limit the base vacuum in the target cell to about $10^{-4}$ Torr. Significant

TABLE VI. (Continued).

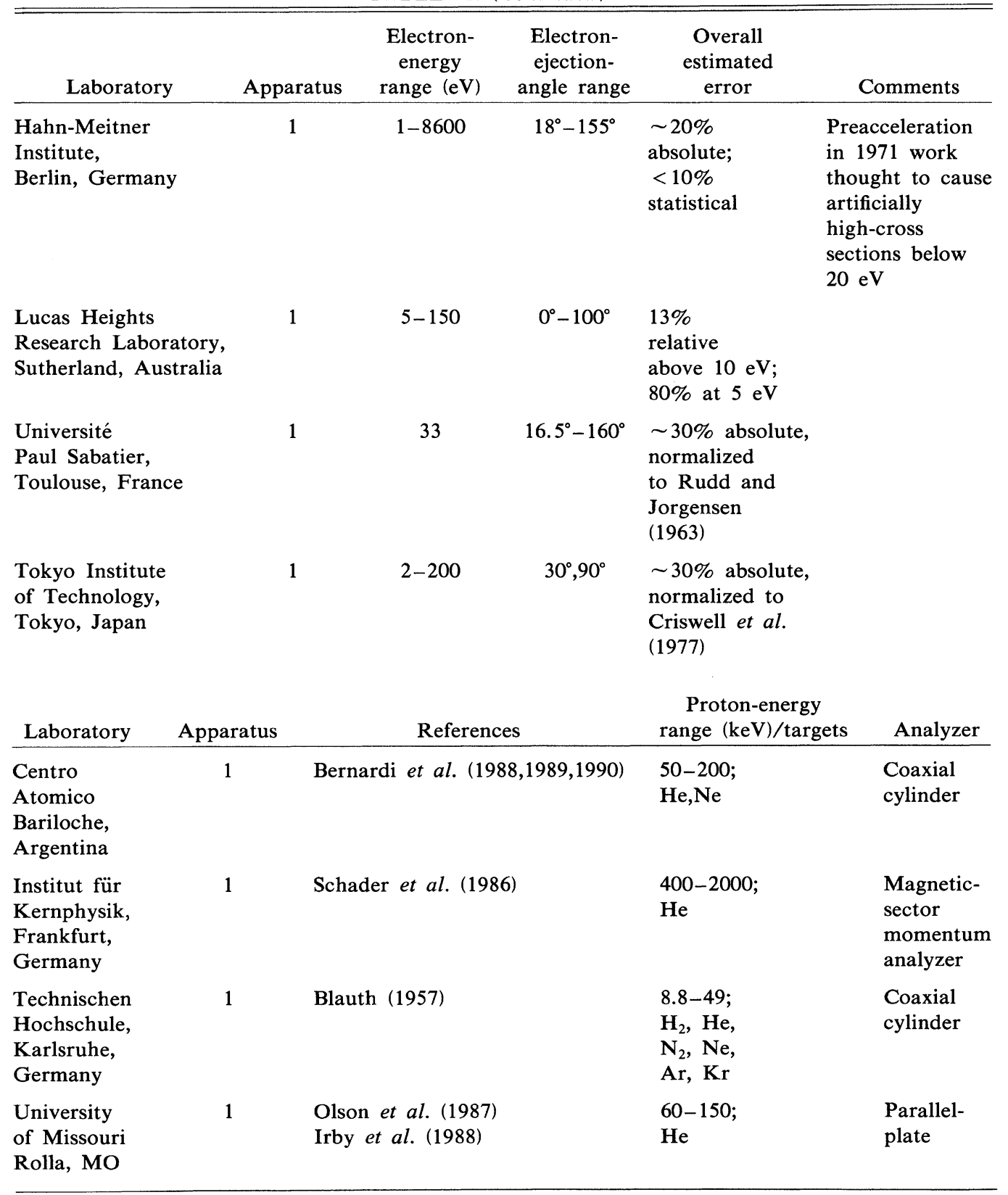


electric charge can also build up on their insulating surfaces, making measurements at low ejection energies unreliable.

A second type of target has been developed by Toburen and co-workers (e.g., Toburen, 1971, 1974; Toburen and Wilson, 1972; Criswell et al., 1977), in which the target gas is introduced into a relatively small triangular target cell (Fig. 15). A narrow slit is cut at the apex of the cell, through which the proton beam passes and ionized electrons escape. In such a target, the gas is well localized and the analyzer/detector operates at correspondingly lower pressures.
In the work of Oda and co-workers (Sataka et al., 1979; Tokoro and Oda, 1985), Gibson and Reid (1984, 1985, 1986, 1987a, 1987b; Fig. 16), and some of the work of Toburen et al. (e.g., Toburen and Wilson, 1975; Lynch et al., 1976; Criswell et al., 1977; Fig. 17), and Stolterfoht et al. (1971a, 1971b; Gabler, 1974), a gas beam target was used. In these cases, the proton beam passes in close proximity to the surface of a multicapillary array (which is either made of metal or has a conducting coating on its surface), through which the target gas is effusing. The gas beam is directed immediately into a pump. Atomic densities in such cases are typically an or-

TABLE VI. (Continued).

\begin{tabular}{|c|c|c|c|c|c|}
\hline Laboratory & Apparatus & Target type & $\begin{array}{c}\text { Detector } \\
\text { type }\end{array}$ & $\begin{array}{l}\text { Quoted } \\
\text { magnetic } \\
\text { fields }(T)\end{array}$ & $\begin{array}{l}\text { Analyzer } \\
\text { pre- or post- } \\
\text { acceleration }\end{array}$ \\
\hline $\begin{array}{l}\text { Centro } \\
\text { Atomico } \\
\text { Bariloche, } \\
\text { Argentina }\end{array}$ & 1 & $\begin{array}{l}\text { Gas beam } \\
\text { from single } \\
\text { capillary }\end{array}$ & CEM & $\begin{array}{l}<7 \times 10^{-7} \\
\text { single } \\
\text { current } \\
\text { coil }\end{array}$ & Post only \\
\hline $\begin{array}{l}\text { Institut für } \\
\text { Kernphysik, } \\
\text { Frankfurt, } \\
\text { Germany }\end{array}$ & 1 & $\begin{array}{l}\text { Gas beam } \\
\text { from single } \\
\text { capillary }\end{array}$ & CEM & $\begin{array}{l}\text { Not } \\
\text { specified }\end{array}$ & $\begin{array}{l}\text { Not } \\
\text { specified }\end{array}$ \\
\hline $\begin{array}{l}\text { Technischen } \\
\text { Hochschule, } \\
\text { Karlsruhe, } \\
\text { Germany }\end{array}$ & 1 & Static & $\begin{array}{l}\text { Geiger- } \\
\text { Müller } \\
\text { tube }\end{array}$ & $\begin{array}{l}\text { Not } \\
\text { specified }\end{array}$ & None \\
\hline \multirow{2}{*}{$\begin{array}{l}\text { University } \\
\text { of Missouri, } \\
\text { Rolla, MO }\end{array}$} & 1 & $\begin{array}{l}\text { Gas beam } \\
\text { from single } \\
\text { capillary } \\
\text { array }\end{array}$ & CEM & $\begin{array}{l}<10^{-6} \\
\text { Magnetic } \\
\text { shield plus } \\
\text { Helmholtz } \\
\text { coils }\end{array}$ & Post only \\
\hline & Apparatus & $\begin{array}{l}\text { Electron- } \\
\text { energy } \\
\text { range }(\mathrm{eV})\end{array}$ & $\begin{array}{l}\text { Electron- } \\
\text { ejection- } \\
\text { angle range }\end{array}$ & $\begin{array}{l}\text { Overall } \\
\text { estimated } \\
\text { error }\end{array}$ & Comments \\
\hline $\begin{array}{l}\text { Centro } \\
\text { Atomico } \\
\text { Bariloche, } \\
\text { Argentina }\end{array}$ & 1 & $5-300$ & $0^{\circ}-90^{\circ}$ & $15 \%$ relative & $\begin{array}{l}\text { Integrated } \\
\text { totals } \\
\text { normalized } \\
\text { to Rudd et al. }(1985)\end{array}$ \\
\hline $\begin{array}{l}\text { Institut für } \\
\text { Kernphysik, } \\
\text { Frankfurt, } \\
\text { Germany }\end{array}$ & 1 & $20-7000$ & $0^{\circ}-60^{\circ}$ & $30 \%$ absolute & $\begin{array}{l}\text { Normalized to } \\
\text { Rudd et al. } \\
(1976,1979)\end{array}$ \\
\hline $\begin{array}{l}\text { Technischen } \\
\text { Hochschule, } \\
\text { Karlsruhe, } \\
\text { Germany }\end{array}$ & 1 & $2-1000$ & $54.5^{\circ}$ & Not specified & $\begin{array}{l}\text { First measurements of } \\
\text { proton DDCS; } \\
\text { significant } \\
\text { background for } \\
\text { electron } \\
\text { energies } \\
\text { above } 100 \mathrm{eV}\end{array}$ \\
\hline $\begin{array}{l}\text { University } \\
\text { of Missouri, } \\
\text { Rolla, MO }\end{array}$ & 1 & $5-150$ & $17^{\circ}-90^{\circ}$ & $\begin{array}{l}10 \% \text { relative; } \\
\text { normalized to } \\
\text { Rudd and } \\
\text { Jorgensen } \\
(1963)\end{array}$ & $\begin{array}{l}\text { Apparatus of } \\
\text { Arcuni and } \\
\text { Schneider } \\
(1987)\end{array}$ \\
\hline
\end{tabular}




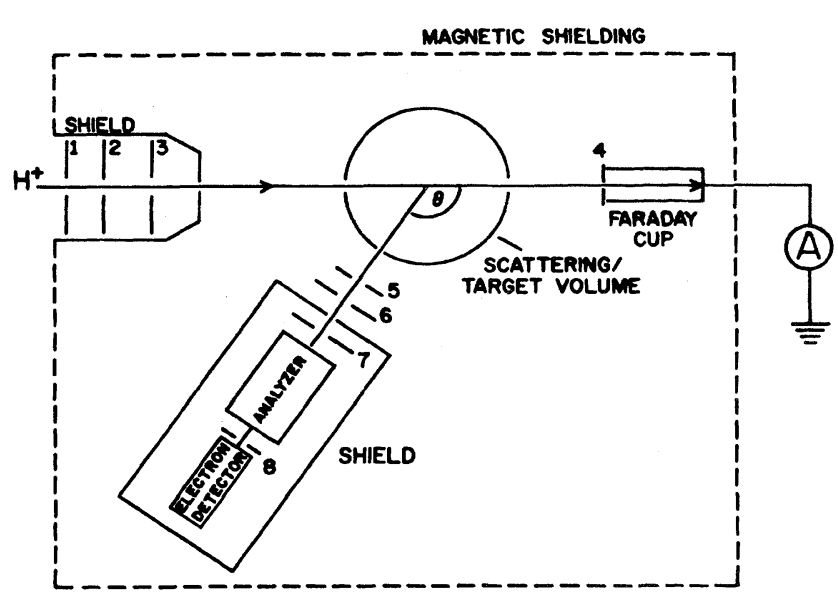

FIG. 12. Schematic diagram of generic apparatus employed in measurements of doubly-differential cross sections for electron ejection: 1 and 2, collimators; 3, electron-suppression aperture (Faraday shield is indicated); 4, Faraday cup and shield; 5 and 6 , solid-angle defining analyzer entrance slits; 7 , preanalyzer acceleration slit; 8 , predetection acceleration slit.

der of magnitude higher in the region where the gas and proton beams intersect than in the surrounding vacuum chamber. This results in relatively high electron production rates without attendant high pressure in the analyzer region and without the potentially disturbing influence of target cell exit apertures. Measurements using the gas

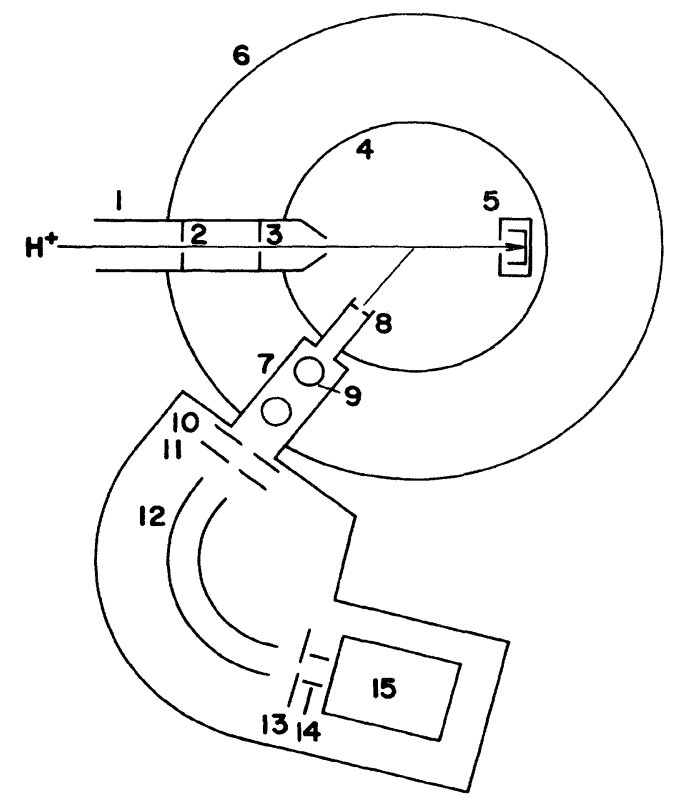

FIG. 13. Schematic diagram of apparatus used by Rudd and co-workers and described in detail by Rudd and Jorgensen (1963): 1, proton entrance port; 2, collimator; 3, electron suppressor; 4, inner gas-containment cylinder; 5, Faraday cup and shield; 6 , outer vacuum-chamber wall; 7 , analyzer entrance tube; 8, differential-pumping and solid-angle-defining aperture; 9, differential pumping ports; 10 , analyzer entrance and solidangle-defining slit; 11 , preacceleration slit; 12 , cylindrical analyzer; 13, analyzer exit slit; 14, detector focusing electrode; 15 , electron multiplier.

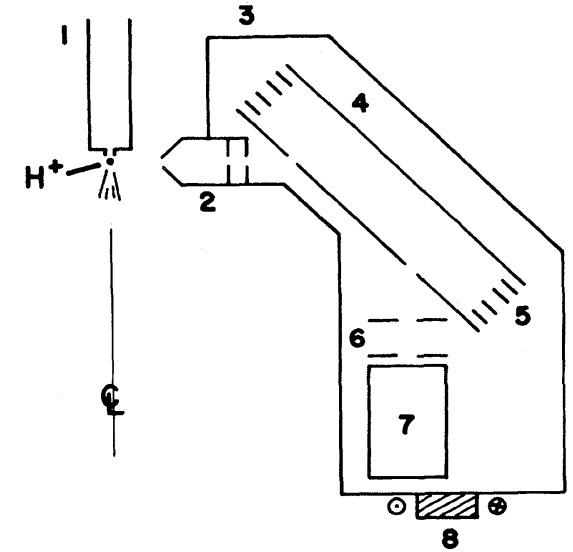

FIG. 14. Schematic diagram of the apparatus used by Stolterfoht et al. (1976, 1979): 1, Effusive gas-target beam; 2, preanalysis acceleration grids; 3 , electron shield/analyzer housing; 4, parallel-plate analyzer; 5, fringe-field correlation electrodes; 6, spurious-electron discriminator grids; 7, electron multiplier; 8, ring for rotation about center line of analyzer assembly. The indicated proton beam is emerging from the plane of the diagram.

beam technique, however, are difficult to put on an absolute scale due to the uncertainty in the target densitylength product $n l$ (see next section). Stolterfoht (1971a, 1971b) and Gabler (1974), as well as Bernardi et al. (1988, 1989, 1990), Schader et al. (1986), and Olson et al. (1987) used a single capillary to produce effusive atomic targets.

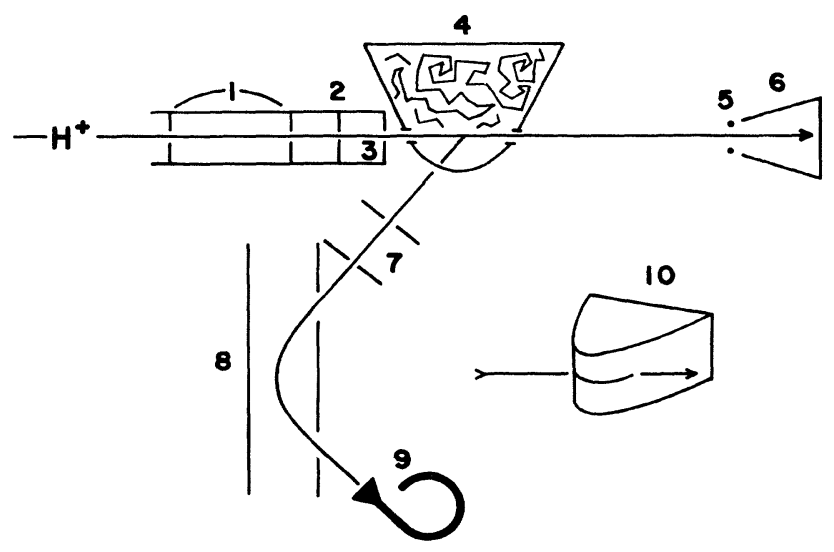

FIG. 15. Schematic diagram of apparatus described by Toburen (1971): 1, collimating apertures; 2, electron-suppression electrode; 3, Faraday shield; 4, triangular target cell showing crumpled high-transmission mesh acting as an electron trap. The segment below the beam line represents a slit through which ejected electrons may leave the target chamber. 5 and 6 , Faraday cup with electron-suppression electrode; 7, solidangle-defining apertures; 8, cylindrical-mirror analyzer with back electrode formed from wire for background reduction; 9 , channel-electron multiplier; 10 , isometric projection of the target cell, showing detail of electron-ejection slit. Arrow represents proton beam. 

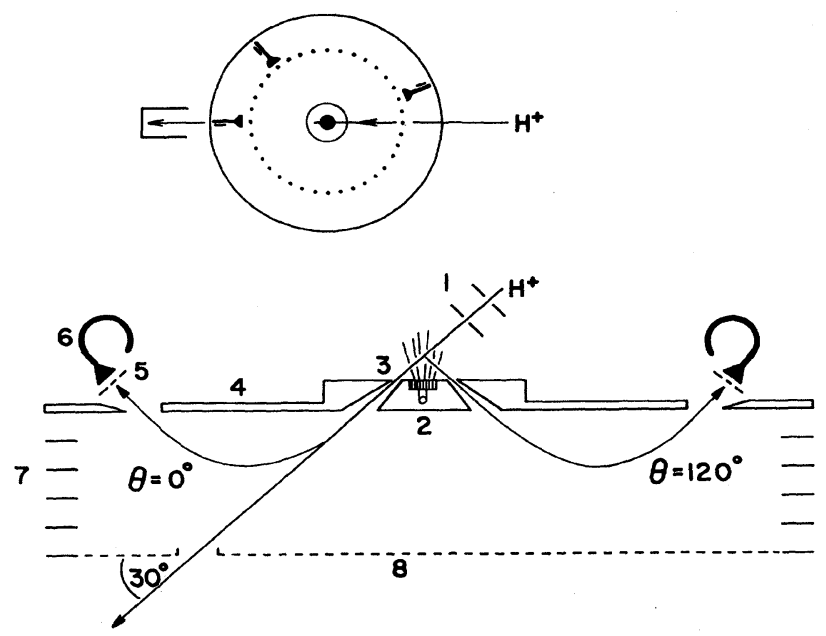

FIG. 16. Schematic diagram of the "fountain" analyzer used by Gibson and Reid (top and side views of the apparatus are shown in the top and bottom of the figure, respectively): 1, ion-beam collimating slits; 2 , effusive gas-target beam produced by a multicapillary array; 3 , analyzer entrance annular slit; 4, analyzer top plate; 5, solid-angle-defining aperture; 6 , channel-electron multiplier (CEM); 7, fringe-field correction electrodes; 8, analyzer back plate made of stainless steel mesh. For high count rates, the $0^{\circ}$ electron detector can be replaced by an electron multiplier. An array of nine CEM's (three of which are shown in the top view) is rotated among 16 angular positions to measure the electron-ejection cross sections.

Having traversed the target region, the proton beam is stopped in a Faraday cup and its current measured. When fast ions are stopped, they can produce both secondary electrons and sputtered ions. At $100 \mathrm{keV}$ incident energy, for example, each proton produces about one electron (see, for example, Thomas, 1985) and $2 \times 10^{-3}$ ions (Andersen and Bay, 1981) with a copper target. Thus, while sputtered ions do not represent a serious problem, the secondary electrons must be prevented from entering the scattering volume. This is accomplished by inserting a negatively-biased element upstream of the

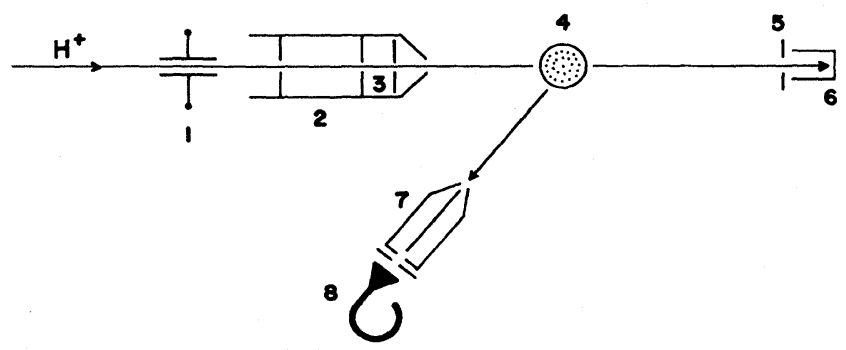

FIG. 17. Schematic diagram of the time-of-flight apparatus described by Toburen and Wilson (1975): $1,40 \mathrm{kV}, 3.33 \mathrm{MHz}$ RF oscillator for beam rastering; 2, collimating apertures and electrostatic shield for electron suppressor; 3, electron suppressor; 4, channelplate gas-beam target (beam is perpendicular to the plane of the diagram); 5, Faraday cup shield; 6, Faraday cup; 7, collimating apertures and accelerating electrode; 8, channelelectron multiplier. beam stop or by biasing the cup positively and shielding it from the interaction region. Other potential problems can be caused by reflected protons (Mashkova and Molchanov, 1985; Thomas, 1985) and proton-induced $\mathrm{x}$ rays or UV photons (e.g., Palmer, Thompson, and Townsend, 1970).

Electrons ejected from the target volume at a given polar angle $\theta$ are energy analyzed and detected. Prior to the analyzer section, the electrons pass through solidangle defining slits, except in the work of Gibson and Reid, which we shall discuss below. Either time-of-flight, magnetic, or electrostatic energy analyzers are used to determine the electron's velocity (Table VI).

Most of the work discussed here has employed $127^{\circ}$, cylindrical-mirror, or parallel-plate electrostatic analyzers, with the exceptions of the time-of-flight analyzer used by Toburen et al. and the magnetic-sector analyzer used by Schader et al. (see Table VI). To avoid uncertainties due to fringing fields in the analyzer, as well as spurious magnetic fields (including that of the earth), electrons are sometimes preaccelerated before entering the analyzer. This is done by running the analyzer section, including the entrance slit, at a small positive bias (typically $+10 \mathrm{~V}$ ) relative to the scattering center. While this procedure can significantly improve the transmission of low-energy electrons through the analyzer, it has the potential disadvantage of producing focusing effects at the analyzer entrance, which can distort the solid-angle acceptance of the device (Kuyatt and Jorgensen, 1963; Rudd and Jorgensen, 1963; Stolterfoht, 1971a, 1971b).

Following passage through the analyzer, the electrons are detected by either a channel-electron multiplier, a discrete-dynode multiplier, or a Geiger-Müller tube (Blauth, 1957). It is very important that the detection efficiency $\epsilon$ of these devices be a well defined function of incident-electron energy. A constant function is the best possibility. To this end, the electrons are generally accelerated by 100 to $400 \mathrm{~V}$ prior to striking the detector. At these energies, $\epsilon$ is relatively insensitive to energy. It is extremely critical that the voltages applied to various elements of the analyzer/detector section, including the high voltage required for operation of the detector, be well shielded from the scattering region.

A novel "fountain" parallel-plate analyzer design, shown in Fig. 16, has been employed by Gibson and Reid $(1984,1985,1986,1987 \mathrm{a}, 1987 \mathrm{~b})$ to measure doublydifferential cross sections. The device consists of two circular plates across which the analyzing voltage is placed. A collimated proton beam crosses a vertically directed multicapillary effusive target, making an angle of $60^{\circ}$ with respect to the analyzer-plate normal. Ionized electrons created in the target region with polar angles relative to the beam direction between $0^{\circ}$ and $120^{\circ}$ enter the analyzer volume through an annular slit. Electrons of the appropriate energy follow trajectories passing through holes evenly spaced on a circle in the top plate, and are detected by an array of channel-electron multi- 
pliers located at several of these exit apertures. Protons leave the bottom plate through an exit hole and are collected in a Faraday cup. This design has the distinct advantage that data for a number of emission angles up to $120^{\circ}$ can be taken simultaneously. In addition, electrons ejected at $0^{\circ}$ can be analyzed. This is more difficult to accomplish with the other configurations we have considered. Unfortunately, the "fountain" design does not allow absolute measurement of cross sections.

For ejection energies less than $10 \mathrm{eV}$, accurate measurements become difficult because of the unpredictable effects of stray electric fields in electrostatic analyzers. To surmount this problem, the technique of time-of-flight velocity analysis has been applied to doubly-differential cross-section measurements by Toburen and Wilson (1975). In their apparatus, shown in Fig. 17, the proton beam is chopped by passing it through deflection plates, across which is placed an oscillating high voltage (typically $\pm 20 \mathrm{kV}$ at $3 \mathrm{MHz}$ ). Proton pulses of duration less than $1 \mathrm{~ns}$ are thus produced as the beam is swept across the collimating slits. Electrons produced in the interaction region pass through two solid-angle defining apertures and are accelerated just prior to being detected by a channel-electron multiplier enclosed in a shielding box. Typical flight times for the electrons are 30-300 nanoseconds, and energies as low as $0.5 \mathrm{eV}$ can be reliably measured, owing primarily to the absence of large electrostatic fields in the analysis region.

The importance of eliminating or minimizing spurious electric and magnetic fields in these measurements cannot be overemphasized. Constant magnetic fields such as the earth's can be reduced to less than $5 \mathrm{mG}$ in the interaction region by orthogonal pairs of Helmholtz coils. Commonly occurring ac magnetic fields, most typically with a frequency of $60 \mathrm{~Hz}$, can be reduced using the same coils with a small ac component added to the dc current. Random or transient magnetic fields can only be eliminated by the use of magnetic shields. In terms of apparatus construction, the use of magnetic materials, such as 400-series stainless steels, must be stringently avoided. Electric patch fields can be minimized by using titanium and molybdenum in electron-optical components and by gold plating apertures through which electrons will pass. Gold plating is especially useful, since it eliminates the formation of surface oxides, which can become electrostatically charged, thus deflecting electron trajectories.

\section{B. Ion energy-loss spectroscopy}

In this method, the occurrence of ionization is determined by analysis of the proton's energy loss, as opposed to the detection of an emitted electron. The only measurements using this technique which have been reported were for an atomic-hydrogen target (Park et al., 1977) and a He target (Park and Schowengerdt, 1969b). The apparatus, developed over a number of years by Park and co-workers (Park and Schowengerdt, 1969a, 1969b; Park et al., 1976, 1978), is shown schematically in Fig. 18. A

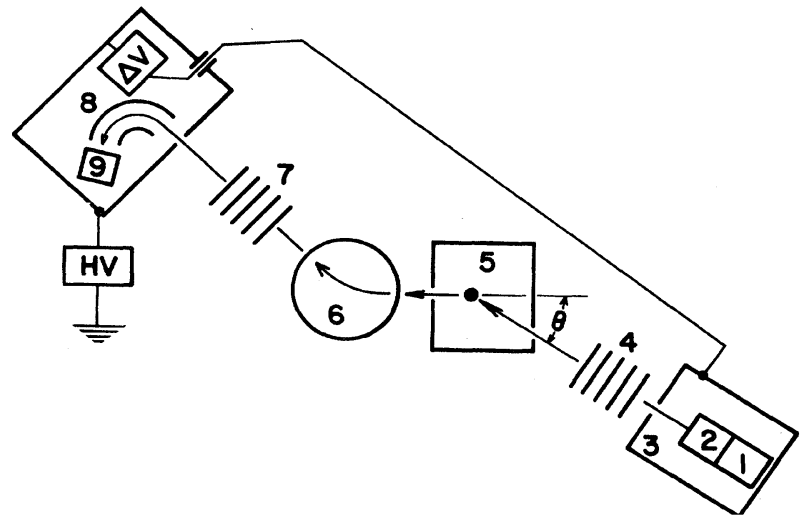

FIG. 18. Schematic diagram of the ion energy-loss spectrometer described by Park et al. (1977): 1, Colutron ion source; 2, Wien filter for mass selection; 3, accelerator high-voltage terminal; 4, accelerator column; 5 , target center, about which the entire accelerator assembly can pivot; 6 , analyzing magnet; 7 , decelerator column; 8 , decelerator high-voltage terminal containing cylindrical electrostatic analyzer, $\Delta V$ high-voltage supply, which is varied to take an energy-loss spectrum; and 9, the electron-multiplier ion detector.

collimated beam of protons is produced with very low $(\sim 1 \mathrm{eV})$ energy spread in a Colutron ion source. It traverses a room-temperature target cell or one that is resistively heated to dissociate molecular hydrogen (Park et al., 1983). The scattered beam is deflected by an analyzing magnet to separate the post-collision charge states. Scattered protons are then decelerated to an energy of $2 \mathrm{keV}$ and detected after passing through a $127^{\circ} \mathrm{cy}-$ lindrical analyzer. In a typical run, an energy-loss spectrum is taken by changing the acceleration voltage by an amount $\Delta V$ and then recording the detected proton count rate after the analyzer as a function of $\Delta V$. In this way, all magnetic and electrostatic elements following the accelerator are kept constant. To be detected, i.e., to be decelerated to precisely the electrostatic analyzer pass energy of $1 \mathrm{keV}$, the proton must lose an amount of energy $\Delta W=e \Delta V$ in the scattering volume. By measuring the count rate for detected protons as a function of $\Delta V$ above the target's ionization limit, one can extract the singly-differential cross section for proton-induced ionization. Electron-capture processes are excluded by the analyzing magnet.

The ion energy-loss method has two general advantages over the electron-analysis technique. First, because the high-energy massive proton is the particle being analyzed, the measured cross sections are much less susceptible to spurious fields. Second, since the proton scattering angles are small, essentially the entire beam is analyzed, and singly-differential cross sections are determined directly. This eliminates potential systematic errors caused, for example, by angle-dependent solid-angle acceptances or absorption coefficients (see next section).

Cross-section information obtained in ion energy-loss spectroscopy is not directly comparable with that ob- 
tained by analysis of the ejected electrons, except in the case of atomic hydrogen. For other targets, proton energy can be lost simultaneously to excitation and/or molecular dissociation.

\section{ERROR ANALYSIS}

\section{A. Analysis of ejected electrons}

The absolute differential cross section for electron emission with energy $W$ into a polar angle $\theta$ is obtained from experimental measurements by using the following equation:

$$
\frac{d^{2} \sigma(W, \theta)}{d W d \Omega}=\frac{\left[N_{S} \exp \left(\sigma_{s} n x\right)-N_{B}\right]}{N_{0} \epsilon t \Delta W(n l \Omega)_{\mathrm{eff}}} .
$$

In this expression, $N_{S}$ and $N_{B}$ are the number of total signal and background counts, respectively, $\sigma_{s}$ is the total scattering cross section for ejected electrons of energy $W, n$ is the number density of target atoms, and $N_{0}$ is the number of incident protons which produce the signal $N_{S}$. The energy spread of electrons (with mean energy $W$ ) passed by the analyzer with a transmission coefficient $t$ is $\Delta W$, and they are detected with efficiency $\epsilon$. The "effective integral of solid angle times path length," $(n l \Omega)_{\mathrm{eff}}$, is the $\theta$-dependent product of differential proton path length times local target number density times subtended solid angle of detection, integrated over all proton path segments viewed by the detector. Finally, $x$ is the effective path length between the collision volume and the detector, given by

$$
x=\int_{0}^{L} \frac{n(\tau)}{n} d \tau
$$

where $L$ is the length of the geometric unscattered electron trajectory, $\tau$ is the distance from the collision volume to a given point along that trajectory, and $n(\tau)$ is the gas density at that point. Note that Eq. (51) is equivalent to Eq. (1), but states explicitly the experimental quantities that must be taken into account.

Each of these quantities must be carefully measured or calculated for an accurate absolute measurement; relative measurements do not require absolute values of $N_{0}, \Delta W$, $\epsilon, t$, or $(n l \Omega)_{\text {eff }}$ We now consider the determination of each of these quantities in detail, and the corresponding experimental systematic errors that can affect the ultimate absolute or relative accuracy of the cross-section values. Table VI gives an overview of the experimental parameters associated with apparatusses used by various investigators to study ejected electrons.

\section{Total signal and background counts, $N_{S}$ and $N_{B}$}

The number of counts registered by the electrondetection circuit for a given number of incident protons is $N_{S}$. The background signal $N_{B}$ results from any process other than simple ejection and subsequent detection of electrons from target-gas atoms caused by the incident protons. If count rates are high and the dead time of the detection circuit is not corrected for, or if the electrons are deflected by spurious electric and magnetic fields, $N_{S}$ can be too low. The only papers that mention dead-time corrections are those of Rudd and Jorgensen (1963) and Rudd and Madison (1976), who applied a maximum correction of $7 \%$ for this effect. Later measurements are presumably less affected by dead-time loss than earlier ones, since the effective pulse-pair resolution of standard counting electronics has improved significantly over the last three decades.

Stray electric fields due to contact potential differences, patch effects, and the proximity of charged insulating surfaces, as well as magnetic fields due to the earth or magnetized materials, are ubiquitous (see, for example, Moore et al., 1983). They become particularly serious when electron trajectories pass close to objects such as defining slits and analyzer surfaces. Spurious electric fields are generally minimized by the use of proper materials and the scrupulous avoidance of insulating components within the line of sight of electron trajectories. Insulating layers of diffusion pump oil can be particularly troublesome in this regard. Effective vacuum pump trapping is thus important and appears to have been adequately considered in all the work reported here. While it is difficult to assess the effects of such electric fields on individual data sets, we note that the results of Kuyatt and Jorgensen (1963) are probably affected by these problems more than those of other workers due to their use of a "zipper" chamber containing large areas of greased rubber surfaces. The data of Schader et al. (1986), taken with a magnetic-field analyzer, are restricted to electron energies above $20 \mathrm{eV}$. The use of a time-of-flight analyzer by the Pacific Northwest Laboratories (PNL) group significantly reduces residual field problems because of its relatively open structure. Field effects can also be reduced by accelerating the electrons before they enter the analyzer. This procedure must be undertaken carefully, however, because it can make $(n l \Omega)_{\text {eff }}$ difficult to calculate (see below).

Most researchers used Helmholtz coils to eliminate the spatially large-scale ac and dc magnetic fields in their chambers. The experiments of Olson et al., Oda et al., Stolterfoht and Gabler, and Toburen and co-workers, involving time-of-flight measurements or electrostatic analysis with proton energies $\leq 100 \mathrm{keV}$, used shields to minimize the magnetic field in the chamber. Such shields reduce variable as well as steady-state fields. Stolterfoht used an electron gun to prove that his electron measurements were not affected by magnetic fields within his experimental error. In all the experiments discussed in this review, the residual magnetic fields were reported to be $<20 \mathrm{mG}$. In a typical experiment, e.g., that discussed by Stolterhoft (1971a, 1971b), such a field could deflect a $5-\mathrm{eV}$ electron by as much as $1 \mathrm{~cm}$ over its path, causing, in principle, a significant systematic error.

We note that both dead-time corrections and the 
effects of spurious fields are most important for lowenergy electron measurements. This is true in the former case because of the relatively high cross sections, and subsequently large count rates, associated with lowenergy electron production.

The background signal can be separated into beamrelated and beam-unrelated counts. The latter category includes "dark counts" from the electron multiplier and noise associated with its attendant electronic circuitry. Both of these signals are easy to isolate and subtract assuming they are time independent. Elimination of beam-related background is generally much more difficult. Such counts can be due to ejection of electrons from fast $\mathrm{H}$ or $\mathrm{H}^{-}$created upstream in the beam line or by direct ionization of the target by $\mathbf{H}$ or $\mathbf{H}^{-}$. Electrons ejected from the target by spurious photons, secondary electrons created in slit scattering, or protons reflected by the Faraday cup also cause beam-related background, as do ultraviolet photons created in the target. Finally, secondary scattering of electrons emitted from the target by chamber walls or residual gas adds to the background. (Direct emission of electrons from the residual gas or secondary emission from slits also contributes but is easily subtracted.)

The formation of neutral- and negative-ion contaminants can be reduced by maintaining good vacuum in the beam line preceding the chamber and by reducing the target volume (gas-beam targets are best for this). Typical beam-line pressures of $2 \times 10^{-6}$ Torr and lengths of 2 $\mathrm{m}$ yield neutralization fractions of less than $1 \%$ for a $10^{-16} \mathrm{~cm}^{2}$ cross section. The Nebraska group mentions corrections for beam neutralization starting with Crooks and Rudd (1971); Criswell et al. (1977), Sataka et al. (1979), Bordenave-Montesquieu et al. (1982), Schader et al. (1986), and Bernardi et al. (1988, 1989) also mention the problem. At high energy ( $\geq 200 \mathrm{keV}$ ), it is probable that none of the data are affected by this problem due to the small neutralization and negative-ion formation cross sections.

Electrons created in the target by $\mathbf{H}, \mathbf{H}^{-}$, photons, secondary electrons, or backscattered protons are essentially indistinguishable from the "real" signal. In general, their numbers can be shown to be negligible (Cheng et al., 1989b). Photons created in the target by the incident beams, however, are a demonstrably serious source of background, and have been observed by Rudd et al. (1966). In that work, photon detection was minimized by coating the back plate of the parallel-plate analyzer they used with nonreflecting colloidal graphite. Ultimately, this background was subtracted from the signal by eliminating the electron counts with a large analyzer voltage (to sweep the electrons away from the detector entrance aperture) and subsequently determining the photon count rate alone.

Potentially the most serious cause of background is the scattering of ejected electrons by target and residual gas and by chamber walls. While background due to direct production of electrons in the ambient gas can be elim- inated by simply removing the target gas and measuring the count rate with beam on, no equivalent procedure exists for the elimination of secondary-scattering background. A quadratic dependence of signal on target pressure would constitute evidence for the scattering of electrons from ambient or target gas, while scattering from walls would exhibit a linear pressure dependence. Most workers mention investigating the pressure dependence of their signals and finding it to be linear after correcting for absorption effects (see below).

To reduce background, several approaches have been taken. The Nebraska group coated their chamber walls with colloidal graphite and added baffles to impede scattered electrons. These precautions produced no significant differences from previous data (Rudd et al., 1966; Cheng et al., 1989b). The Pacific Northwest Laboratories group crumpled fine wire mesh and inserted it directly behind the proton beam in their target cell to prevent reflected electrons from leaving the cell (see Fig. 15). Both the cylindrical-mirror analyzer used by the PNL group and the "fountain" analyzer of Gibson and Reid used back plates made of wire mesh to minimize electron reflection. Stolterfoht and Gabler enclosed their analyzer in a tight box with negatively biased grids placed across the pumping ports. They also used discriminator grids in front of their electron multiplier to eliminate low-energy multiply scattered electrons.

\section{Number density of target gas, $n$}

The target density is an important factor in both the absorption correction term, $\exp \left(\sigma_{s} n x\right)$, and $(n l \Omega)_{\text {eff }}$. In all the work reported here, it is determined from the target pressure $P$, measured either with an ionization gauge or a capacitance manometer. These devices are in turn calibrated by a McLeod gauge or, in some of the cases involving capacitance manometers, a direct dead-weight force measurement performed by the manometer's manufacturer. Care must be taken to account for the effects of thermal gradients and finite conductance between the point of measurement and the target region (Knudsen, 1910; Blaauw et al., 1980). Calibration measurements using a McLeod gauge must also avoid error due to inadvertent gas pumping by streaming mercury vapor. [These considerations have been discussed in detail by Rudd et al. (1985); see also Schram et al., 1965, and references therein.] In their later work Rudd and co-workers used a differential capacitance manometer with a correction for nonzero reference pressure. Toburen initially checked his capacitance manometer readings by placing a second manometer directly at the target cell, and found his calculated pressure differential to be accurate.

Target pressures in these experiments range between $4 \times 10^{-5}$ and $3 \times 10^{-2}$ Torr. No quadratic pressure dependence of the results is reported. Error estimates for the measurement of the pressure range between 5 and 
$12 \%$. Absolute knowledge of the pressure is irrelevant, of course, for relative measurements, but relative values must be known precisely and must be reproducible.

In the early experiments at Nebraska, great care was taken to purify target gases. In more recent work, these precautions have been relaxed somewhat, with the simple use of research purity gas and all-metal pressure regulators and transfer lines.

\section{The "absorption" coefficient $\sigma_{s} x$}

When electrons are ejected from the target by incident protons, they can be prevented from entering the electron multiplier by scattering from another gas atom. "Target gas" in this context refers to any gas atom that is in the chamber as a result of opening the target-gas valve; these atoms need not be in a volume intersected by the proton beam. "Absorption" of the ejected electrons can thus occur at any point along their trajectory prior to striking the first surface of the detector. The absorption process is of course closely related to secondary scattering. One detector's uncounted, absorbed electron is another detector's background. If $N$ counts would be observed by a detector in the absence of absorption, $N_{S}$ is given by $N_{S}=N \exp \left(-\sigma_{s} n x\right)$, where $\sigma_{s}$ is the total scattering cross section, which is dependent on electron energy and target-gas species. (In principle, $\sigma_{s}$ should be taken as an "effective" scattering cross section that does not include the contributions of forward scattering into the detector solid angle.) Generally, $\sigma_{s}$ decreases with increasing electron energy. The effective path length $x$ is given by Eq. (52).

The absorption factor can either be measured or calculated. Stolterfoht observed an exponential decrease in detected electron count rate with increasing target-gas pressure, independent of proton energy or ejection angle, when he used a static target. He was thus able to show a maximum absorption of $30 \%$ for $1-\mathrm{eV}$ electrons and could experimentally correct his data for this effect. The Nebraska and Pacific Northwest Laboratories groups have calculated the $\exp \left(-\sigma_{s} n x\right)$ factor using data for the total cross sections compiled in various references (Brüche, 1927; Normand, 1930; Golden and Bandel, 1965; Golden et al., 1966). Such calculations are difficult, especially for the PNL target, given the complicated vacuum geometry of the slits, tubes, and apertures that surround the electron trajectory. The PNL group found upon making this correction (which was occasionally as large as $25 \%$ ), however, that the measured crosssection values were rendered independent of pressure for various angles and electron energies, indicating that the correction had been made properly.

Bordenave-Montesquieu et al. ran at sufficiently low pressures $\left(<10^{-4}\right.$ Torr $)$ that absorption corrections could be neglected.

The absorption correction has been neglected in the case of effusive gas-beam targets. This neglect is probably justifiable, given the much better localization of tar- get gas and the generally lower values of $n$ at the target center in this case.

\section{Number of protons $N_{0}$}

This quantity is measured by integrating the proton charge collected in the Faraday cup and dividing by the proton charge. Errors in this factor can result from electron capture downstream from the target region by the protons, failure to eliminate secondary-electron emission from the Faraday cup, faulty charge integration, failure to capture all of the incident beam in the cup, and reflection of protons from the cup back into the interaction region. These last two problems are most severe for low proton energies ( $\lesssim 10 \mathrm{keV}$ ), where large-angle scattering is more probable and susceptibility to stray magnetic fields is greater. Potential errors from these effects can be checked by varying the Faraday cup position and the size of its entrance aperture and by steering the beam electrostatically into the center of the cup (Kuyatt and Jorgensen, 1963; Rudd and Jorgensen, 1963).

The problem of beam neutralization by the target gas is more severe for static targets, but it is mentioned only by Rudd and co-workers after 1971, and by BordenaveMontesquieu et al. Attempts to eliminate secondaryelectron emission from the cup must be made with care, since fields from suppressor grids can leak into the interaction region (Rudd and Madison, 1976). Faulty integration of the proton current is unlikely to cause errors larger than a few percent; Toburen and Cheng, Rudd, and Hsu have calibrated their ammeters with standard current sources. The question of proton reflection has not been addressed, except by Rudd, who finds the effect to be negligible at $50 \mathrm{keV}$. Sputtering effects should also be negligible.

\section{Integrated path length/solid angle $(n I \Omega)_{\mathrm{eff}}$}

Accuracy in this term depends critically on knowledge of the target-gas pressure profile over the incident-beam path, and the solid-angle acceptance of the analyzer. It is given by

$$
(n l \Omega)_{\mathrm{eff}}(\theta)=\int \Omega(z, \theta) n(z) d z,
$$

where the integral is over the proton-beam path, $d z$ is an element of length along that path, and $\Omega$ is the solid angle subtended by the analyzer and detector for a given path element and electron-ejection angle. For the static gas targets of Rudd et al., Stolterfoht et al., and Brodenave-Montesquieu et al., $n(z)$ is a constant over the acceptance window of the analyzers for all angles investigated. Thus $(n l \Omega)_{\mathrm{eff}}=n \int \Omega(z, \theta) d z$. This integral, a purely geometric quantity, has been evaluated in terms of slit sizes and positions by Kuyatt (1968). The pressure profile of the PNL target is complicated by the longitudinal slit in the target cell. This group has determined $n(z)$ by using the same procedure used to calculate $x$. They 
experimentally verified the accuracy of the calculation by measuring $\mathrm{K}$-Auger electrons from $\mathrm{N}_{2}$ as a function of $\theta$. Since these electrons are emitted isotropically, properly corrected data will have no dependence on $\theta$, as was found to be the case. For relative measurements, only knowledge of the functional form of $n(z)$ is important.

The use of gas-beam targets essentially precludes the possibility of absolute measurements because of the uncertainty in the beam density. Moreover, unless the length of the beam path viewed is so small that $n(z)$ is constant for all $\theta$, or is so large that the entire proton path in the target is viewed by the analyzer at any angle, even relative measurements will suffer some angular systematic error. This problem is not discussed by any of the researchers using gas-beam targets except Gibson and Reid and may be a source of significant error. In this regard, we mention the systematic differences in the measured angular dependence of doubly-differential cross sections between Gibson and Reid and other workers (see, specifically, Gibson and Reid, 1986, and Cheng et al., 1989b). It appears that the poorly characterized shape of the effusive gas target in Gibson and Reid's "fountain" spectrometer allows a significant angular dependence of $(n l \Omega)_{\text {eff }}$ that was not accounted for in the analysis. Interestingly, the angular discrepancies in the DDCS measurements do not yield corresponding discrepancies in the SDCS, as will be seen in Sec. $X$.

The problem of determining $d \Omega$ is also difficult. The effective solid angle subtended by the detector can be altered by spurious fields and by focusing effects due to intentional preacceleration of the ejected electrons prior to their entrance into the analyzer (Kuyatt and Jorgensen, 1963). The effect of preacceleration on solid-angle acceptance (as well as energy resolution) has been considered extensively by Stolterfoht, Rudd, Toburen and coworkers, who find it to be most important for electron energies $<10 \mathrm{eV}$ (Manson et al., 1975). The finite diameter of the proton beam must also be considered in calculating the solid angle. All of the proton beams in the experiments reported here were $\leq 2 \mathrm{~mm}$ in diameter.

We note that estimates in the uncertainty in $(n l \Omega)_{\text {eff }}$ are typically of the order of $10 \%$, meaning that this factor contributes significantly to the overall uncertainty of the cross-section measurements.

\section{Energy width-transmission product of the analyzer, $\Delta W t$}

If we consider illumination of the entrance slit of an aberration-free electrostatic analyzer with electrons uniformly distributed in energy and angle (within the acceptance angle of the device), the output distribution of electrons versus their energy will be either trapezoidal or triangular in shape, with the peak of the distribution centered on the mean pass energy $W$. The base and top widths of the distribution depend only on the geometric dimensions of the analyzer (Rudd, 1972). The full width at half-maximum of the distribution is then defined as
$\Delta W$, and its height taken to be the "transmission" $t$. Thus, for a "white" incident-electron flux of $j$ electrons per unit energy interval, the analyzer will pass $j \Delta W t$ electrons. Remarkably, the area under the transmission curve does not change even when angular aberration effects are considered (Kuyatt, 1968; Rudd, 1972). Since $\Delta W t$ is a purely geometric quantity, it can be calculated. Toburen (1971) has measured $\Delta W$ by investigating Auger electrons; Rudd and Jorgensen (1963), Kuyatt and Jorgensen (1963), and Stolterfoht (1971a, 1971b) have used electron guns to verify their calculated values of $\Delta W$.

\section{Detection efficiency $\epsilon$}

This factor represents the percentage of electrons that, upon traversing the analyzer, produce recorded pulses. It includes the transmission of the discriminator/pulsecounting circuit and can be measured in a number of ways. The first absolute determination of $\epsilon$ in work of this type was reported by Rudd and Jorgensen (1963). They replaced their analyzer and detector with a Faraday cup and measured the electron flux emitted from the target at a given angle. By integrating the data taken with the electron multiplier and analyzer in the pulse-counting mode over electron energy, and comparing this result with the Faraday-cup value, they extracted $\epsilon$ with an uncertainty of about $10 \%$. A similar technique, described by Stolterfoht (1971a), Rudd and Madison (1976), and Cacak and Jorgensen (1970) involves replacement of the target by a defocused electron gun. The electron flux entering the analyzer is determined either by measuring the emission current from the thermionic filament directly or by measuring the current density from the gun through a larger aperture of well-known area using a Faraday cup. In the latter case, a smaller aperture is subsequently placed in front of the analyzer and the electron flux is counted directly. These procedures also yield uncertainties in $\epsilon$ of about $10 \%$.

Toburen (1971) directly calibrated a channeltron for incident-electron energies of $600 \mathrm{eV}$ by comparing the channeltron count rate with that of a windowless-flow proportional counter operated in the Geiger region, whose efficiency was taken to be unity. Cheng et al. (1989a) have described a novel method for determining the discriminator transmission and electron-multiplier efficiency separately. The integral pulse-height spectrum from the counting circuit is obtained and a linear fit to the region of minimum slope is made. The zero-pulseheight intercept of this fit is divided into the value of the spectrum at the discriminator setting used for measurements, and the ratio is taken as the discriminator transmission. In addition, the efficiency of the multiplier is obtained by measuring its gain, which is used to infer the gain per dynode stage. If the electron-emission process at each dynode is assumed to obey Poisson statistics, the probability of no electrons reaching the collector anode, equal to $1-\epsilon$, is easily calculated.

Gibson and Reid used several channeltrons and a 
discrete-dynode electron multiplier simultaneously to detect scattered electrons. While they did not attempt to make absolute measurements, a knowledge of the relative efficiencies of their various detectors was required to provide accurate angular distributions. This was accomplished by rotating the detector array in situ to measure various parts of the angular distribution with several detectors, and cross-correlating the relative count rates at a given angle.

One possible cause of serious systematic error lies in the variation of $\epsilon$ with incident-electron energy. Some researchers appear to have neglected this problem, although knowledge of this dependence is crucial even for relative cross-section measurements. In general, the dependence must be determined experimentally, as has been done in some of the experiments discussed here. The variation of $\epsilon$ can be minimized by accelerating the electrons to $\sim 100 \mathrm{eV}$ after passage through the analyzer. The efficiency vs energy curve above this voltage is relatively flat for both channeltrons and discrete dynode devices; variations of $\sim 10 \%$ are typical between 500 and $2000 \mathrm{eV}$ (see, for example, Bordoni, 1971; Toburen, 1971).

In all cases discussed above, determinations of $\epsilon$ have yielded values between 0.27 and 0.98 . This range is due primarily to the different types and conditions of the detectors themselves, although some variation due to the incident secondary-electron energy has been observed. Both relative and absolute uncertainties between about $5 \%$ and $10 \%$ have been quoted.

Finally, we note that space charge of the positive ionized core of target gas can, in principle, cause systematic variations in the energy of the ejected electrons. Such effects can be significant if the differential cross section is falling rapidly with electron energy. For the experiments reported here, typical beam currents $(\sim 1 \mu \mathrm{A})$ would result in negligible electron-energy shifts.

\section{B. Ion energy-loss spectroscopy}

In the technique developed by Park and co-workers, the singly-differential cross section $d \sigma / d W$ is measured directly and is given by the equation

$N_{S}(W)-N_{B}(W)=n l N_{0} \int_{0}^{\infty} d \sigma / d W^{\prime} \Phi\left(W-W^{\prime}\right) d W^{\prime}$,

where $N_{S}$ and $N_{B}$ are the number of protons detected with energy loss $W$, with and without target gas, respectively, for an incident number of protons $N_{0} ; n$ and $l$ are the target number density and length; and $\Phi(W)$ is the energy profile of the incident beam (Park and Schowengerdt, 1969b; Park et al., 1977).

Implicit in Eq. (54) are two assumptions. First, no correction for multiple scattering of the proton is included. This correction has been experimentally demonstrated to be small in both the $\mathrm{He}$ and the $\mathrm{H}$ experiments. Second, $d \sigma / d W$ is taken to be the complete, angle- integrated singly-differential cross section. For this assumption to be correct, essentially all of the protons scattered in the target must ultimately be detected. Park et al. were able to demonstrate complete angular acceptance in their measurements of total cross sections with He by showing that the sum of the energy-loss, elastic, and charge-transfer signals was equal to the incidentproton flux within their measurement uncertainty. This observation was consistent with theoretical calculations showing that protons scattered to angles greater than the instrumental angular acceptance of $\sim 10^{-3}$ rad would not affect the integrated cross section. It should be pointed out, however, that the fraction of protons scattered outside a given angle of acceptance is generally a function of $W$. Thus failure to attain complete angular acceptance in regions of $W$ where the SDCS does not contribute significantly to the total cross section would not affect the above summation, but would result in systematic errors in the cross sections for those values of $W$. The report of the later $H$ measurements (Park et al., 1977) does not mention angular acceptance checks. With the caveat that the smaller singly-differential cross sections could, in principle, have some systematic error due to incomplete angular acceptance, there is strong circumstantial evidence to indicate at least the total cross sections do not suffer from this problem (Park, 1983).

If $d \sigma / d W$ varies slowly with $W$, as it does in the smooth energy-loss ionization continuum, then $\Phi(W)$, which varies rapidly about its central energy, can be taken as a $\delta$ function in Eq. (54), yielding

$$
d \sigma / d W=\left[N_{S}(W)-N_{B}(W)\right] / n l N_{0} .
$$

Several important issues relating to the quantities in Eq. (55) are as follows.

\section{The beam-related signal-background difference $N_{S}-N_{B}$}

The ion energy-loss signal includes, in general, processes other than those contributing to the electron signal discussed in the preceding section, except for $\mathbf{H}$ targets. In addition to simple single ionization, electron-analysis experiments will detect electrons resulting from multiple ionization and from transfer ionization that yields fast neutral hydrogen. The ion energy-loss experiments detect not only simple multiple ionization in addition to single ionization, but also energy loss resulting from simultaneous target excitation and/or dissociation. Moreover, collisions with more than one electron ejected are weighted more heavily in the electron-analysis method than single ionization [Rudd et al. (1985), p. 966]. Thus cross sections measured by the two methods are comparable only when multiple and transfer ionization, as well as excitation and dissociation, are negligible. Typically, such cross sections are at least an order of magnitude smaller than those for single-electron ejection (see, for example, DuBois, 1985, 1986 and DuBois and Manson, 1987, and references therein). 
The background signal $N_{B}$ includes contributions from background gas (most typically $\mathrm{N}_{2}$ ) and reflections of the incident beam from the back (positive voltage) cylinder segment of the analyzer. With $\mathrm{H}$ targets, it is also necessary to eliminate the signal from undissociated $\mathrm{H}_{2}$ in the target oven. This is done indirectly by determining the ratio of the energy-loss signals at 10.2 and $12.5 \mathrm{eV}$, corresponding to excitation of $H(n=2)$ and of the Lyman $\alpha$ bands of $\mathrm{H}_{2}$, respectively. This ratio yields the target dissociation fraction and allows a correction for $\mathrm{H}_{2}$ ionization. The dissociation fraction for $\mathbf{H}$ is reported by Park et al. to be better than $97 \%$, so this correction is small.

\section{The target length-density product $n l$}

The target length-density product $n l$ is difficult to determine with $\mathrm{H}$ targets, due to the high temperatures and open structure of the $\mathbf{H}$ furnace. As a result, the $\mathbf{H}$ results of Park et al. have been integrated and subsequently normalized to the Born approximation for the total ionization cross section at $200 \mathrm{keV}$. A long, differentially pumped target cell with small entrance and exit apertures has been used in the He work, and $n l$ can be calculated to a high degree of accuracy (Park and Schowengerdt, 1969b).

\section{The number of incident protons $N_{0}$}

This number is measured by integrating the incident ion beam over its energy spread. Since the beam is detected by the complete "detector" following the target cell, as is the product signal $N_{S}$, all factors regarding detector efficiency, angular acceptance, and ion-related secondary-electron detection cancel out in the ratio of Eq. (54). This canceling of factors is a significant advantage of the ion energy-loss method.

\section{RECOMMENDED VALUES OF SINGLY-DIFFERENTIAL CROSS SECTIONS}

The tables of double-differential cross-section data that have accumulated are quite voluminous. For example, Toburen's data for one target gas at a single proton energy typically involved measurements at 200-300 electron energies for each of 11 angles. Even the singlydifferential cross-section data are rather extensive when many combinations of proton energy, electron energy, and target are involved. Generally only a small fraction of the data appears in published form, and even the tables that have been published (Rudd et al., 1976,1979) contain condensations or selections of the data.

For the purposes of this review, we have obtained tables of the original data from most of the authors. While data from different laboratories are in good general agreement, there are areas of disagreement. Intercomparison of many data sets indicates systematic variations with proton and electron energy and target, which enable us to choose among divergent data.

The presentation of recommended values of singlydifferential cross sections in tabular form is difficult because the pertinent ranges of electron energies change with proton energy. An electron-energy range for which the cross section is changing slowly at one proton energy may be one where it is varying rapidly at a different proton energy.

These three problems, the large volume of data, the discrepancies among different data sets, and the difficulty of presenting data in tabular form, are solved in this review by the use of semiempirical models. Model parameters are determined by fitting the models to experimental data. Averaging and/or selection were done with respect to the parameters rather than the original data. As a result, the recommended singly-differential cross-section values are expressed in the form of reasonably simple equations with a small number of parameters.

\section{A. Choice of model}

As discussed in Sec. VI, there are several semiempirical models to choose from, each having its own advantages and disadvantages. For instance, Kim's model is able to reproduce many details in the singly-differential cross sections of Ar and $\mathrm{N}_{2}$ for a limited range of $T_{0}$, and the resulting SDCS's can serve as normalization standards for experiments. For most practical applications, however, a model is needed that reproduces only gross features of the SDCS over a wide range of primary energies, since experimental data are available from $5 \mathrm{keV}$ to $5 \mathrm{MeV}$. The only model presently available that represents the energy distributions over the entire range of primary as well as secondary energies is the one proposed by Rudd (1987, 1988). The parameters in this model must be determined from experimental data. Sufficient data are presently available for this determination for ten of the common gases. The model has been successfully fitted to all of these targets, thus determining its parameters.

The Rudd model reproduces gross features of the singly-differential cross sections of all targets reasonably well for low and intermediate energy collisions. It is also accurate at high energies for single-shell targets such as $\mathrm{He}$ and $\mathrm{H}_{2}$, but the form of the equation is not flexible enough to reproduce the fine details of SDCS's in different multishell targets. For quantitative reproduction of $\sigma(W)$ at $T_{0} \geq 300 \mathrm{keV}$, models that incorporate individual details of the dipole term are preferred. As described earlier, parameters for the model proposed by Kiim are available only for argon and nitrogen.

\section{B. Data adjustment}

Kim's model depends mostly on the dipole function [Eq. (24)] and on the binary-encounter term [Eq. (35)] to reproduce the global shape of singly-differential cross 
sections. Only parts of the experimental data that exhibit the expected shape are used to determine the mixture of dipole and nondipole contributions, to avoid distortions that may be introduced by uncertain parts of the experimental results. Thus it is unnecessary to modify any experimental data to determine adjustable parameters in Kim's model. On the other hand, the parameters in Rudd's model are sensitive to the shape and magnitude of the original data, and it is desirable before parameters are fitted to modify data deemed to be seriously inaccurate. We describe below how some of the experimental results were adjusted before applying Rudd's model.

Since total ionization cross sections have generally been determined to a higher accuracy than differential ones, and since recommended values of these total cross sections are readily available (Rudd et al., 1985), each experimental set of singly-differential cross sections was first adjusted to be consistent with the total cross sections. Two methods, both one-parameter adjustments, were used. In the first method, all SDCS's in a set were adjusted by a single multiplicative factor. In the second method, most of the adjustment was made on the larger low-energy cross sections. The equation used in this method was

$$
\sigma(W) / \sigma_{u}(0)=\left[1+K_{c} \sigma_{u}(W) / \sigma_{u}(0)\right]^{1 / K_{c}}-1,
$$

where $\sigma(W)$ is the adjusted cross section, $\sigma_{u}(W)$ is the uncorrected cross section, and $\sigma_{u}(0)$ is its uncorrected value at the lowest measured secondary energy, generally $2 \mathrm{eV}$. The value of $K_{c}$ was varied until the adjusted cross section yielded an integral equal to the recommended total cross section.

An example of this second form of adjustment is shown in Fig. 19. In (a) the original, unadjusted data are shown from two experiments. The agreement is quite good above about 20 or $30 \mathrm{eV}$, but the run of Crooks and Rudd (1971) yields an integral smaller than the recommended total cross sections of Rudd et al. (1985). The discrepancy appears due to the falloff of the cross section

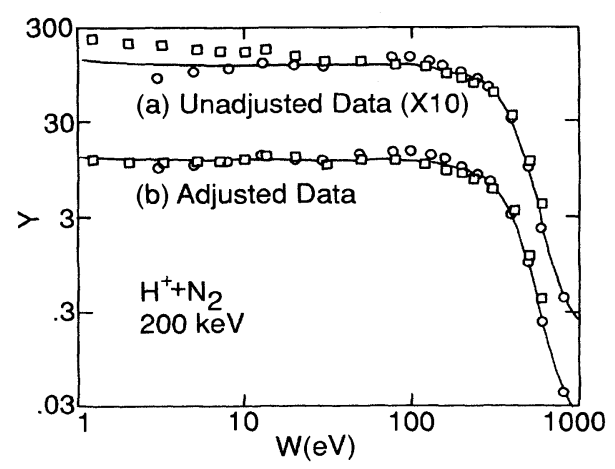

FIG. 19. Plot of electron-energy distributions from 200-keV $\mathrm{H}^{+}+\mathrm{N}_{2}$ to show the effect of data adjustment: $\square$, data of Stolterfoht (1971b); $O$, data of Crooks and Rudd (1971); solid line, Rudd model. (a) Original, unadjusted data. (b) Data adjusted by Eq. (56). at the lowest energies. On the other hand, the data of Stolterfoht (1971b) integrate to too high a value, most likely because of cross sections that are too high below about $20 \mathrm{eV}$. When Eq. (56) is applied to the two sets of data and the values of $K_{C}$ are chosen to give the correct integrated value, the resulting adjusted data are brought into much better agreement, as shown in Fig. 19(b).

Each of the runs of the data analyzed in this paper was subjected to the adjustment procedure of Eq. (56) before fitting. An exception was made for the data of Toburen and his collaborators. In their data, time-of-flight analysis was used to improve the low-energy accuracy, so that the relative uncertainty was virtually constant over the entire range. Therefore the first method of adjustment was used. Except for a few runs at low proton energies, the adjustments in the data were small.

\section{Fitting of the Rudd model}

A nonlinear least-squares program was adapted from the CURFIT program of Bevington (1969). The three parameters $F_{1}, F_{2}$, and $\alpha$ of the Rudd model were varied to provide the best fit for each energy spectrum at a given proton energy. As discussed in Sec. VI.D, the values of $F_{1}$ and $F_{2}$ for all subshells with $I<2 I_{1}$ were determined from Eqs. (43) and (44) using the same set of target parameters. For the inner subshells a single set of parameters was used for all targets. The inner-shell parameters were difficult to determine with any accuracy, but approximate values were sufficient, since inner shells generally do not contribute much to the overall cross sections. These values were estimated by fitting at the highenergy tails of the energy distributions, where inner-shell contributions predominate. Also, the known total cross sections for inner-shell ionization were used. If differential cross-section data for specific inner shells becomes available in the future, the inner-shell parameters can be determined more accurately.

The values of $\alpha$ were found to be independent of proton energy within experimental uncertainty and were taken to be constant for a given target. The parameters $F_{1}$ and $F_{2}$ vary smoothly with proton energy, allowing fits by the functions in Eqs. (43)-(48). There appear to be two maxima in the graph of $F_{1}$ for several of the targets, as seen in the example in Fig. 20.

\section{Table of parameters in the Rudd model}

The values of the target parameters for Eqs. (43) and (44) are given in Table V. It can be seen that the values of $A_{1}$ are not far from unity, and the sums $A_{1}+A_{2}$ are not far from 2, as expected on the basis of the Bethe theory. The parameter $\alpha$ always falls between 0.5 and 0.9. The parameters $C_{1}$ and $C_{2}$, which determine the magnitudes of $F_{1}$ and $F_{2}$ at low proton energies, are typically in the range of 0.2 to 1 . The values of $D_{1}$ and $D_{2}$, which determine the slope of the cross sections with pro- 


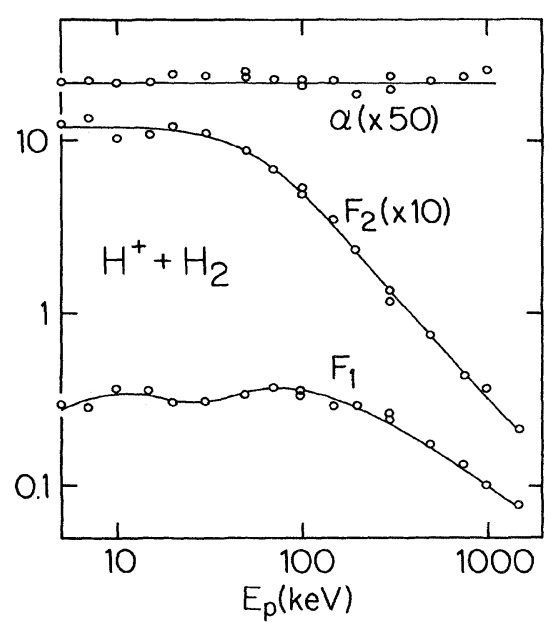

FIG. 20. Plot of the parameters $F_{1}, F_{2}$, and $\alpha$ obtained in fitting the data for $5-1500 \mathrm{keV} \mathrm{H}{ }^{+}+\mathrm{H}_{2}$ collisions. Data from Rudd (1979), Rudd and Jorgensen (1963), Rudd et al. (1966), and Toburen and Wilson (1972). The lines for $F_{1}$ and $F_{2}$ are the best fits to Eqs. (43) and (44). The line for $\alpha$ is a constant equal to the average of the fitted values.

ton energy at low energies, have a greater variation, even becoming negative for some targets. The parameters $B_{1}$, $B_{2}, E_{1}$, and $E_{2}$ are less easily interpreted and have greater variations, since they merely determine connections between the low- and high-energy regions.

\section{E. Recommended values}

Having fitted the model to the data for ten different target gases, it is possible to calculate cross sections for any combination of primary and secondary energy for any of those targets from parameters of the model Eq. (41). The following is a sample computation for the case of $8-\mathrm{eV}$ electrons ejected in $\mathrm{H}^{+}+\mathrm{He}$ collisions at 50 keV. With $I=24.6 \mathrm{eV}$ and $N=2$, we have $w=0.325$, $v=1.05$, and $S=2.15 \AA^{2}$. From the parameters in Table II, we have $L_{1}=0.389, H_{1}=0.232, L_{2}=0.718$, and $H_{2}=5.65$, giving $F_{1}=0.621$ and $F_{2}=0.637$. The cutoff energy is $w_{c}=4 v^{2}-2 v-R / 4 I=2.19$, whence $\exp \left[\alpha\left(w-w_{c}\right) / v\right]=0.219$. Moreover, $\quad\left(F_{1}+F_{2} w\right) /$ $(1+w)^{3}=0.356$. The cross section is then $0.0255 \AA^{2} / \mathrm{eV}$. For comparison, integrated experimental results of Rudd and Madison (1976) and Rudd and Jorgensen (1963) are 0.0341 and $0.0251 \AA 2 / \mathrm{eV}$, respectively; Gibson and Reid's value (interpolated) is $0.024 \AA^{2} / \mathrm{eV}$.

Additional samples of recommended cross sections are given in Table VII for selected combinations of primary and secondary energies with hydrogen $\left(\mathrm{H}_{2}\right)$ as a target. Table VIII gives the total and partial cross sections for the various subshells of argon at $300-\mathrm{keV}$ proton energy. Figure 21 shows these data compared with the results of Toburen et al. (1978). The same quantities are plotted as $Y$ in Fig. 22.

At the cost of somewhat greater complexity, the Kim

TABLE VII. Sample of recommended cross sections (in $\AA^{2} / \mathrm{eV}$ ) for $\mathbf{H}^{+}+\mathrm{H}_{2}$ calculated from Eq. (41). The notation 5.52(-2) denotes $5 \times 10^{-2}$.

\begin{tabular}{|c|c|c|c|c|c|c|}
\hline \multirow[b]{2}{*}{$W(\mathrm{eV})$} & \multicolumn{6}{|c|}{$T_{0}$} \\
\hline & $10 \mathrm{keV}$ & $30 \mathrm{keV}$ & $100 \mathrm{keV}$ & $300 \mathrm{keV}$ & $1000 \mathrm{keV}$ & $3000 \mathrm{keV}$ \\
\hline 0 & $5.52(-2)$ & $9.19(-2)$ & $1.24(-1)$ & $7.78(-2)$ & $3.47(-2)$ & $1.50(-2)$ \\
\hline 1.5 & $5.12(-2)$ & $8.98(-2)$ & $1.06(-1)$ & $6.24(-2)$ & $2.71(-2)$ & $1.16(-2)$ \\
\hline 2 & $4.94(-2)$ & $8.84(-2)$ & $1.01(-1)$ & $5.83(-2)$ & $2.51(-2)$ & $1.07(-2)$ \\
\hline 3 & $4.56(-2)$ & $8.48(-2)$ & $9.25(-2)$ & $5.11(-2)$ & $2.17(-2)$ & $9.25(-3)$ \\
\hline 5 & $3.76(-2)$ & $7.64(-2)$ & $7.73(-2)$ & $4.02(-2)$ & $1.66(-2)$ & $6.99(-3)$ \\
\hline 7 & $3.04(-2)$ & $6.79(-2)$ & $6.55(-2)$ & $3.24(-2)$ & $1.30(-2)$ & $5.43(-3)$ \\
\hline 10 & $2.15(-2)$ & $5.61(-2)$ & $5.23(-2)$ & $2.43(-2)$ & $9.46(-3)$ & $3.88(-3)$ \\
\hline 15. & $1.17(-2)$ & $4.06(-2)$ & $3.76(-2)$ & $1.62(-2)$ & $6.02(-3)$ & $2.42(-3)$ \\
\hline 20 & $6.25(-3)$ & $2.94(-2)$ & $2.82(-2)$ & $1.16(-2)$ & $4.13(-3)$ & $1.63(-3)$ \\
\hline 30 & $1.75(-3)$ & $1.54(-2)$ & $1.75(-2)$ & $6.73(-3)$ & $2.26(-3)$ & $8.66(-4)$ \\
\hline 50 & $1.44(-4)$ & $4.08(-3)$ & $8.58(-3)$ & $3.07(-3)$ & $9.58(-4)$ & $3.51(-4)$ \\
\hline 70 & $1.33(-5)$ & $1.00(-3)$ & $4.95(-3)$ & $1.75(-3)$ & $5.21(-4)$ & $1.85(-4)$ \\
\hline 100 & $4.40(-7)$ & $1.19(-4)$ & $2.49(-3)$ & $9.34(-4)$ & $2.66(-4)$ & $9.23(-5)$ \\
\hline 150 & $1.91(-9)$ & $3.95(-6)$ & $7.98(-4)$ & $4.44(-4)$ & $1.21(-4)$ & $4.09(-5)$ \\
\hline 200 & $9.92(-12)$ & $1.52(-7)$ & $1.83(-4)$ & $2.58(-4)$ & $6.92(-5)$ & $2.28(-5)$ \\
\hline 300 & $3.52(-16)$ & $3.00(-10)$ & $5.36(-6)$ & $1.17(-4)$ & $3.10(-5)$ & $1.00(-5)$ \\
\hline 500 & $7.50(-25)$ & $1.96(-15)$ & $5.03(-9)$ & $3.10(-5)$ & $1.12(-5)$ & $3.57(-6)$ \\
\hline 700 & & $1.76(-20)$ & $6.44(-12)$ & $1.57(-6)$ & $5.74(-6)$ & $1.80(-6)$ \\
\hline 1000 & & & $3.90(-16)$ & $4.58(-9)$ & $2.81(-6)$ & $8.81(-7)$ \\
\hline 1500 & & & $5.26(-23)$ & $3.52(-13)$ & $1.24(-6)$ & $3.89(-7)$ \\
\hline 2000 & & & & $3.40(-17)$ & $3.39(-7)$ & $2.18(-7)$ \\
\hline 3000 & & & & $4.46(-25)$ & $2.17(-11)$ & $9.69(-8)$ \\
\hline 5000 & & & & & $4.40(-20)$ & $3.47(-8)$ \\
\hline 7000 & & & & & & $2.36(-10)$ \\
\hline 10000 & & & & & & $8.40(-18)$ \\
\hline
\end{tabular}


TABLE VIII. Cross sections (in $\AA^{2} / \mathrm{eV}$ ) by subshell for $300 \mathrm{keV} \mathrm{H^{+ }}+$ Ar calculated from Eq. (41). The notation 1.97(-1) denotes $1.97 \times 10^{-1}$

\begin{tabular}{|c|c|c|c|c|c|c|}
\hline$W(\mathrm{eV})$ & $3 p$ & $3 s$ & $2 p$ & $2 s$ & $1 s$ & Total \\
\hline 2 & $1.97(-1)$ & $1.58(-2)$ & $1.44(-4)$ & $1.89(-5)$ & $3.00(-9)$ & $2.14(-1)$ \\
\hline 4 & $1.50(-1)$ & $1.36(-2)$ & $1.42(-4)$ & $1.87(-5)$ & $3.00(-9)$ & $1.64(-1)$ \\
\hline 6 & $1.18(-1)$ & $1.18(-2)$ & $1.39(-4)$ & $1.84(-5)$ & $3.00(-9)$ & $1.30(-1)$ \\
\hline 10 & $7.71(-2)$ & $9.19(-3)$ & $1.34(-4)$ & $1.79(-5)$ & $3.00(-9)$ & $8.64(-2)$ \\
\hline 15 & $4.98(-2)$ & $6.95(-3)$ & $1.29(-4)$ & $1.73(-5)$ & $2.99(-9)$ & $5.69(-2)$ \\
\hline 20 & $3.46(-2)$ & $5.44(-3)$ & $1.23(-4)$ & $1.68(-5)$ & $2.99(-9)$ & $4.01(-2)$ \\
\hline 30 & $1.92(-2)$ & $3.57(-3)$ & $1.13(-4)$ & $1.57(-5)$ & $2.98(-9)$ & $2.29(-2)$ \\
\hline 50 & $8.32(-3)$ & $1.87(-3)$ & $9.64(-5)$ & $1.38(-5)$ & $2.96(-9)$ & $1.03(-2)$ \\
\hline 75 & $4.04(-3)$ & $1.02(-3)$ & $7.96(-5)$ & $1.19(-5)$ & $2.94(-9)$ & $5.16(-3)$ \\
\hline 100 & $2.37(-3)$ & $6.43(-4)$ & $6.65(-5)$ & $1.02(-5)$ & $2.92(-9)$ & $3.09(-3)$ \\
\hline 150 & $1.09(-3)$ & $3.19(-4)$ & $4.76(-5)$ & $7.74(-6)$ & $2.86(-9)$ & $1.47(-3)$ \\
\hline 250 & $3.99(-4)$ & $1.22(-4)$ & $2.61(-5)$ & $4.62(-6)$ & $2.73(-9)$ & $5.52(-4)$ \\
\hline 300 & $2.75(-4)$ & $8.30(-5)$ & $1.98(-5)$ & $3.62(-6)$ & $2.66(-9)$ & $3.81(-4)$ \\
\hline 500 & $6.85(-5)$ & $1.86(-5)$ & $7.05(-6)$ & $1.45(-6)$ & $2.34(-9)$ & $9.56(-5)$ \\
\hline 750 & $2.61(-6)$ & $1.27(-6)$ & $2.10(-6)$ & $4.96(-7)$ & $1.93(-9)$ & $6.48(-6)$ \\
\hline
\end{tabular}

model yields more accurate cross sections for primary energies above $200 \mathrm{keV}$. Parameters for the two targets, argon and nitrogen, are given in Tables II and III for use in Eqs. (32) - (34). The two models by Kim and Rudd are compared with unadjusted experimental data in Figs. 8-11.

\section{$X$. DISCUSSION OF SINGLY-DIFFERENTIAL CROSS-SECTION DATA FOR INDIVIDUAL TARGETS}

Most of the available data (listed in Table IV) for the ten gases were used in the determination of parameters for the Rudd model, as illustrated in Figs. 23-32. Some sets of experimental data were excluded. Blauth (1957) presented doubly-differential cross-section data for helium, neon, argon, krypton, hydrogen, and nitrogen, but only at one angle, $54.5^{\circ}$. It was thus not possible to extract singly-differential cross sections by angular integration. The data of Bordenave-Montesquieu et al. (1973, 1982) yielded angular SDCS's at only one electron ejection energy. The pioneering experiment of Kuyatt and Jorgensen (1963) provided DDCS data for hydrogen but with incorrect normalization, owing to poor collection of

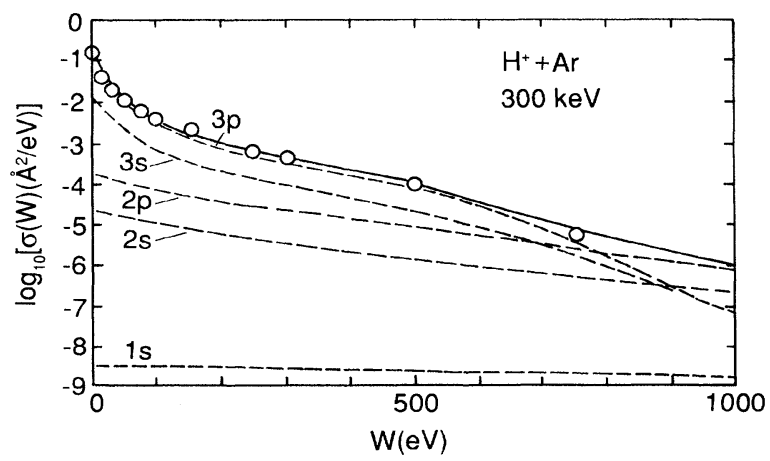

FIG. 21. Partial cross sections (dashed lines) from the subshells of argon, as calculated from Eq. (41), along with the total (solid line) for $300 \mathrm{keV}$. Also shown are the corresponding experimental data from Toburen et al. (1978). low-energy electrons. Measurements of DDCS by Rødbro and Andersen (1979), Sataka, Urakawa, and Oda (1979), Tokoro and Oda (1985), and Olson et al. (1987) are too limited to permit SDCS calculations. The DDCS data of Bernardi et al. (1988, 1989, 1990) and Schader et al. (1986) are probably extensive enough for calculation of SDCS's, but were not published in tabular form. Furthermore, their cross sections were normalized to the data of Rudd et al. (1976).

The only data for atomic hydrogen at the present time are those of Park et al. (1977). Unfortunately, their electron energy range extended only to $20 \mathrm{eV}$, insufficient to provide information about the parameter $\alpha$. Furthermore, the proton energy range is not sufficient to allow unambiguous determination of the parameters in a model that relates high and low energies. For this reason, atomic hydrogen was not included in our analysis and fitting.

\section{A. Helium}

Thirty-eight runs in the range $5-5000 \mathrm{keV}$ from 7 different data sets were fitted. The value of $A_{1}$ from the

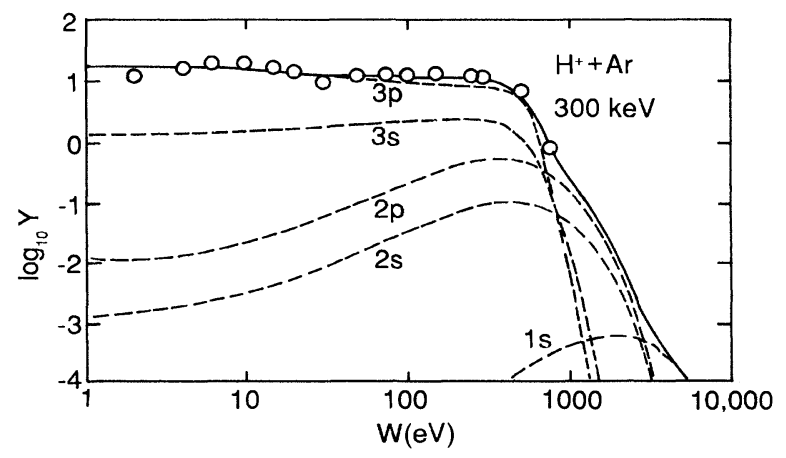

FIG. 22. The same as Fig. 21 plotted as $Y(E, T)$, the ratio of the cross sections to the Rutherford cross section. 
fit was $1.02,15 \%$ higher than the 0.89 value obtained from oscillator-strength data. The values of the parameters in Table $\mathrm{V}$ fit the total cross sections with an average deviation of $3 \%$.

A large downward adjustment was needed in the lowenergy data (especially those of Rudd and Madison, 1976) to match the recommended total cross sections. The charge transfer to the continuum (CTC) peak results in a discrepancy for the runs at the intermediate energies of $40-150 \mathrm{keV}$ in all data sets, the peaks being especially prominent in the data of Gibson and Reid $(1985,1986)$.

There is also a discrepancy near the cutoff energy between the model calculations and the data of Rudd and Jogensen (1963) and of Rudd et al. (1966). This stems from the size of the angular mesh, which was too coarse to reproduce the binary-encounter peak in the experiment at high energies. The data of Toburen (1971) and of Stolterfoht (1971a) taken with smaller angular steps do not have this discrepancy. The data of Park and Schowengerdt (1969b) agrees well with other data just below the cutoff but falls too low at lower energies.

The average overall accuracy of the fit is estimated to be $\pm 10 \%$. Samples of the data are shown in Fig. 23, where $Y$, the ratio of the cross section to the Rutherford

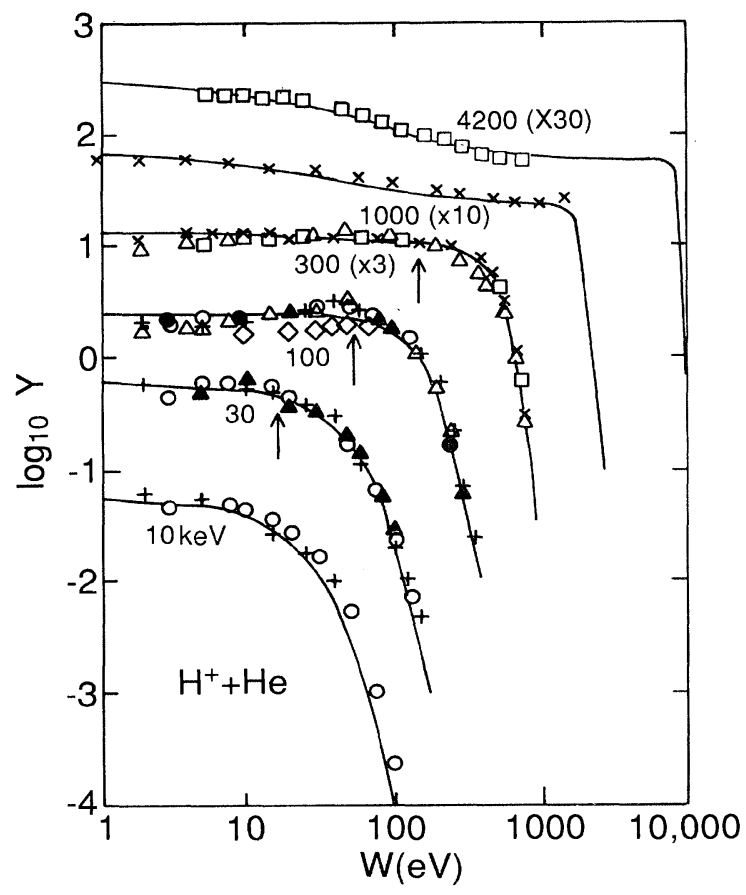

FIG. 23. Energy distribution of secondary electrons from proton collisions on helium, shown as a ratio of SDCS to the Rutherford cross section (see text): $\bigcirc$, Rudd and Madison (1976); Rudd and Jorgenson (1963); $\triangle$, Rudd et al. (1966); $\times$, Toburen et al. (1978); + , Cheng et al. (1989a); $\diamond$, Park and Schowengerdt (1969b); $\square$, Manson et al. (1975); $\boldsymbol{\Delta}$, Gibson and Reid $(1985,1986)$. Lines are the model with parameters given in Table III. Vertical arrows indicate the expected position of the peaks from charge transfer to the continuum. cross section, is plotted against the secondary energy $W$ for various primary energies from 10 to $4200 \mathrm{keV}$.

\section{B. Neon}

Three sets of data combine to cover $T_{0}$ from 7.5 to $1500 \mathrm{keV}$, with a gap between 300 and $1000 \mathrm{keV}$. While the individual runs were fitted fairly well by the model, it was difficult to find parameters that agreed with the singly-differential cross sections and also gave the correct asymptotic total cross sections at high and low energies. The value $A_{1}=0.75$ from the total cross sections is too large to fit the higher-energy SDCS's. The value 0.58 was chosen as the best compromise. The data and fit of the model are shown in Fig. 24.

The discrepancy between the model and the SDCS's due to the CTC peak begins to appear at about $30 \mathrm{keV}$ and is quite prominent at $150-300 \mathrm{keV}$. At $300 \mathrm{keV}$ and above, another peak (in $Y$ but not in $d \sigma / d W$ ) comes at a somewhat lower secondary energy, $W \sim 50 \mathrm{eV}$. This peak, which represents an enhancement of the continuum-dipole interaction, grows as the proton energy is increased (Kim, 1976).

At primary energies of $50-300 \mathrm{keV}$, the data above the cutoff are generally higher than the model. This may result from our arbitrary classification of the $2 s$ subshell as an "inner shell," since $I(2 s)>2 I(2 p)$. If the parameter $A_{1}$ had been given the lower value of 0.57 for the outer shells rather than the larger value $(0.66)$ characteristic of the inner shells, this discrepancy would have been much smaller. Nevertheless, the model should provide SDCS's with an average error of about $15 \%$.

\section{Argon}

Six sets of data cover the energies $5-5000 \mathrm{keV}$. These are shown for representative primary energies in Fig. 25. At low energies the data of Criswell and Toburen (Cris-

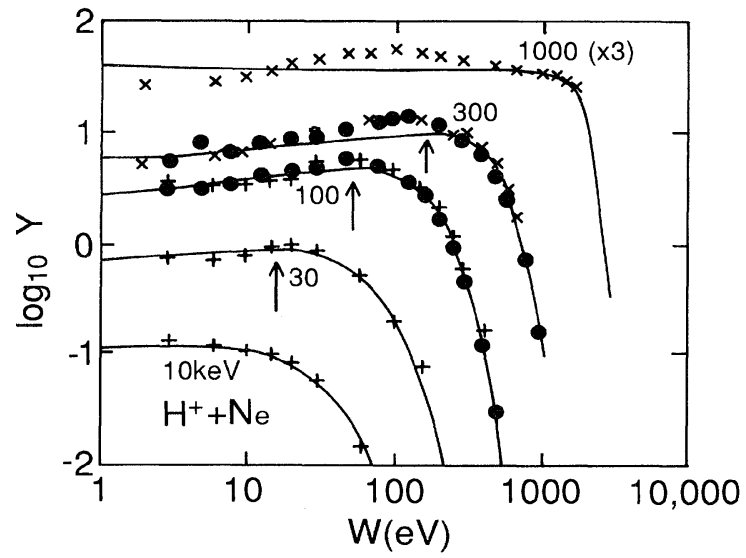

FIG. 24. Energy distribution of secondary electrons from proton collisions on neon: Crooks and Rudd (1971). The rest of legend as in Fig. 23. 


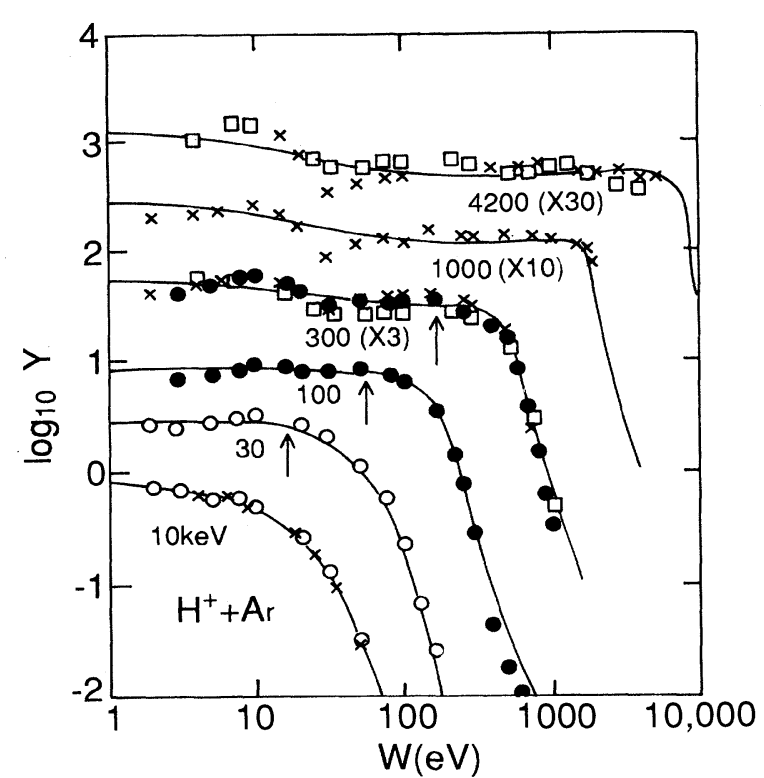

FIG. 25. Energy distribution of secondary electrons from proton collisions on argon: $\bigcirc$, Rudd's data from Criswell et al. (1977); Crooks and Rudd (1971); $\times, 10 \mathrm{keV}$, Criswell's data in Criswell et al. (1977); $\times$, high energies, Toburen et al. (1978); $\square$, Gabler (1974). The rest of legend as in Fig. 23.

well et al., 1977) agree quite well with those of Rudd (1977) after both are adjusted to match the recommended total cross sections. Below $50 \mathrm{keV}$ none of the runs were carried to sufficiently high secondary energies for the $n=2$ shell contribution to be appreciable. The fit of the $3 p$ and $3 s$ subshells to the Rudd model is very good, with an estimated average uncertainty of $10 \%$. Figure 22 shows model calculations for the separate and combined subshells.

In the range of 50 to $500 \mathrm{keV}$ the $n=2$ contribution is apparent beyond the cutoff for the outer shell and may be compared with the Rudd model, where data are available. In some cases the model calculations for the $n=2$ contribution are in good agreement with the data or are slightly lower. However, in most cases the model results beyond the $n=3$ cutoff are too high. For the 50-500 $\mathrm{keV}$ energies the Rudd model yields results that are probably accurate to $10-15 \%$ below the $n=3$ cutoff, but the error increases to about $50 \%$ above this cutoff.

Above $1000 \mathrm{keV}$, the $n=2$ contribution actually exceeds that of the $3 s$ electrons at some energies below the cutoff, and at $2000 \mathrm{keV}$ it begins to exceed that of the $3 p$ electrons. Above $4200 \mathrm{keV}$ the $n=2$ contribution is dominant over a large fraction of the secondary-energy range.

The Cooper minimum appears as a dip in $Y$ at about $30 \mathrm{eV}$ for impact energies greater than $300 \mathrm{keV}$ and is clearly seen in Figs. 22 and 25. The CTC peak is barely noticeable in most of the data and is most prominent in the data of Gibson and Reid (1987a, 1987b).

The accuracy of Rudd's model above $500 \mathrm{keV}$ is es- timated at $20 \%$ except near the Cooper minimum and above the $n=3$ cutoff, where the agreement is worse (see Figs. 8, 9, 22, and 25). For applications that require details of $d \sigma / d W$, Kim's model, discussed in Sec. VI.C, gives a more accurate fit and should be used for $T_{0} \geq 200$ keV (cf. Figs. 8 and 9).

\section{Krypton}

The two data sets for krypton cover $T_{0}$ from 7.5 to $4200 \mathrm{keV}$, with a gap from 150 to $1500 \mathrm{keV}$. However, because the model fits smoothly both the low- and highenergy data sets, the missing energies can be filled in quite accurately and there is little need to take additional data to obtain SDCS's. Only small adjustments to the experimental data were required except at the very lowest proton energies (below $20 \mathrm{keV}$ ); the model fits well nearly everywhere. One exception lies at the highest secondary energy for the two lowest primary-energy runs where the model results are somewhat too high. The other discrepancy occurs in the highest-energy run $(4200 \mathrm{keV})$. The experimental data are suspect here, since their pattern differs abruptly from that of other runs in the same data set.

The model should be accurate to $10-15 \%$ with the exceptions noted. The value of $A_{1}=1.46$ is $12 \%$ higher than the value expected from oscillator-strength data. Figure 26 shows the data for representative energies along with the model.

\section{E. Molecular hydrogen}

Twenty runs from five sets of data were individually fitted with an average deviation of $10 \%$. The value of $A_{1}$ calculated from oscillator strength $(0.80)$ was too small to fit the SDCS data and was replaced by 0.96 . Cross sec-

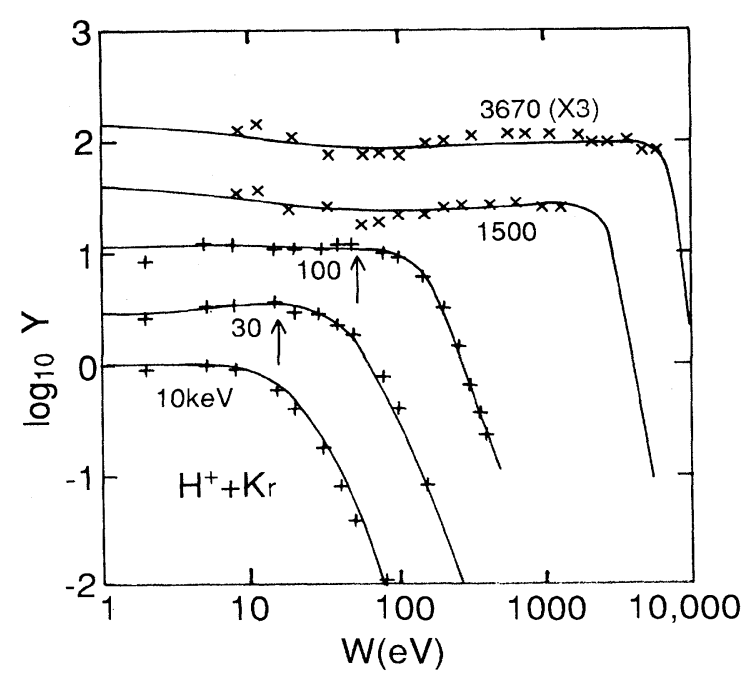

FIG. 26. Energy distribution of secondary electrons from proton collisions on krypton. Legend as in Fig. 23. 
tions computed with the parameters given in the table agreed with the measured data with an average deviation of $15 \%$.

The data by Rudd (1979) required the largest adjustment, especially at the lowest energies, but were fitted well after adjustment. The single run at $50 \mathrm{keV}$ by Gibson and Reid (1987a, 1987b) had an average deviation of $30 \%$ due to the CTC peak. This peak also showed up, but to a lesser extent, in the other data sets. In the Rudd et al. (1966) data, a discrepancy occurs in the 200- and $300-\mathrm{keV}$ runs near the cutoff, owing to the coarseness of the angular mesh, mentioned earlier. A discrepancy between the model and the data of Toburen at $\geq 1000 \mathrm{keV}$ occurs near the cutoff. Since the cutoff is very sharp at high energies, cross sections obtained by integrating data over a limited number of angles are apt to be inaccurate. The cross sections given by the model are estimated to have an accuracy of $10 \%$. Data and the fit are shown in Fig. 27.

\section{F. Molecular nitrogen}

Sets of data from several laboratories over the range of $T_{0}=5$ to $1700 \mathrm{keV}$ are available for nitrogen. The best value for $A_{1}, 1.05$, coincides with that predicted by the oscillator strength. The lowest-energy data would be fitted better with a slightly smaller value of $\alpha$ (say, 0.60 instead of 0.70 ), while some of the runs at higher energies would have benefitted from a somewhat larger value (e.g., $\alpha=0.80$ ). However, the three highest-energy runs did not extend far enough past the cutoff to give any information on $\alpha$.

The cross sections in Stolterfoht's 500-keV run (Stolterfoht 1971b), and to a lesser extent in his 400- and 300$\mathrm{keV}$ runs, tend to drop too fast at energies approaching cutoff, as compared to the corresponding data of To-

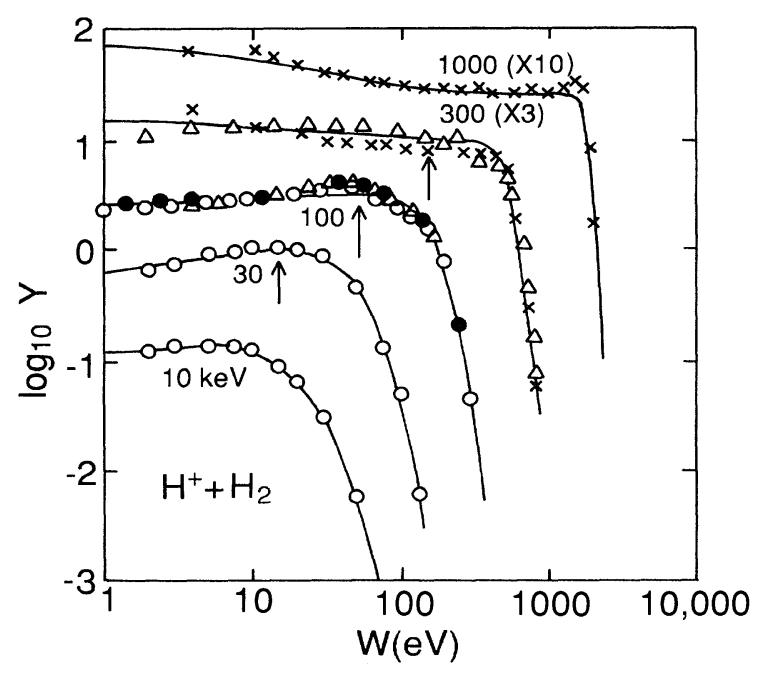

FIG. 27. Energy distribution of secondary electrons from proton collisions on molecular hydrogen; $O$, Rudd (1979); $X$, Toburen and Wilson (1972). Rest of legend as in Fig. 23. buren and Wilson (1975) and of Crooks and Rudd (1971). Toburen's data tend to be lower than the model and the other data sets at low energies. The accuracy of the model is estimated to be $15 \%$, except for low secondary energies, where it is worse. The CTC peak appears from 50 to $300 \mathrm{keV}$ (except in Stolterfoht's data), but at the higher energies it comes at a somewhat lower $W$ than expected.

The $K$-shell contribution is noticeable at $100 \mathrm{keV}$ and above, but is only important above the cutoff for the other shells. The measurements are subject to considerable variation in this region of very small cross sections, but the fit is generally good, as can be seen in Fig. 28 .

As was the case for Ar, Kim's model, discussed in Sec. VI.C, is recommended for applications that require details of $d \sigma / d W$ for $T_{0} \geq 200 \mathrm{keV}$ (cf. Figs. 10 and 11).

\section{G. Molecular oxygen}

Three sets of data cover the range of 7.5 to $300 \mathrm{keV}$. With guidance from total cross sections, the parameters were estimated over the full range of energies. The major discrepancies between model and data were at $v_{e}=v_{p}$ due to CTC. Smaller discrepancies occur at the highest secondary energies. These may be due to a poor set of parameters for the inner shells, but more likely to fluctuations and incorrect background subtraction in the experimental data. The model should be accurate to $15 \%$. Figure 29 shows the results. The value of $A_{1}$ is 1.02 , very close to the 1.04 calculated from the oscillator strength.

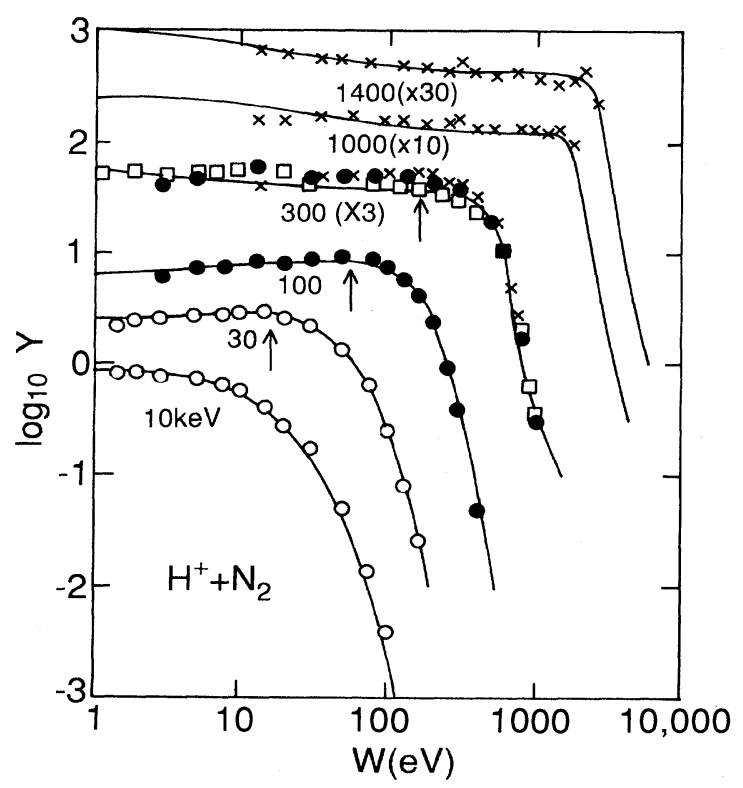

FIG. 28. Energy distribution of secondary electrons from proton collisions on molecular nitrogen: $\bigcirc$, Rudd (1979); $X$, Toburen (1971); $\square$, Stolterfoht (1971b). Rest of legend as in Fig. 23. 


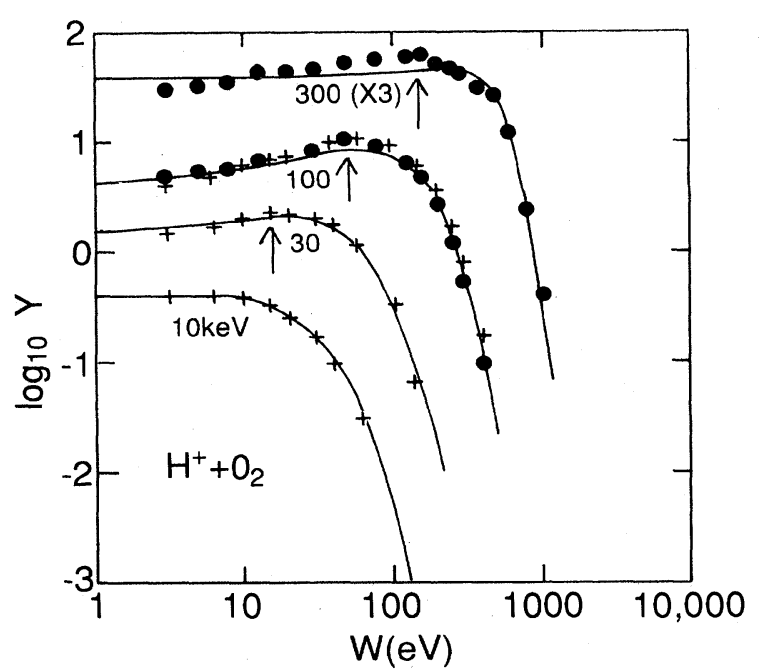

FIG. 29. Energy distribution of secondary electrons from proton collisions on molecular oxygen: + , Cheng et al. (1989b); Crooks and Rudd (1971). Rest of legend as in Fig. 23.

\section{H. Carbon dioxide}

Few data are available for this target, namely, those of Cheng et al. (1989b) and the single run at $50 \mathrm{keV}$ by Gibson and Reid (1987a, 1987b). Parameters determined from those data can nevertheless be extrapolated, with guidance from total cross sections, to yield reasonably reliable SDCS's over a much wider range of energies. Fitting each run individually yields an average deviation of $9 \%$, which increases to $14 \%$ when the parameters are determined by Eqs. (43)-(48). Part of this deviation is due to the CTC peak, which appears at $30 \mathrm{keV}$ and above. Otherwise, most of the deviation represents random scatter. None of the data sets required a large adjustment to achieve consistency with the recommended total cross sections. Results are shown in Fig. 30. The value 1.09 for $A_{1}$ is about $4 \%$ lower than that required to fit the total cross sections (1.13). The value calculated from the oscillator strength is still lower, namely, 0.93 .

\section{Water vapor}

The data for water vapor comprise 11 runs from $T_{0}=15$ to $1500 \mathrm{keV}$. Those by Bolorizadeh and Rudd (1986) at low proton energies show a leveling off at high secondary energies which is probably spurious. This high-energy tail was ignored in the fitting. A relatively small adjustment was needed to bring the various runs into agreement with the recommended total cross sections. The average deviation of the individual fits from the model was $9 \%$ and rose only to $10 \%$ for parameters calculated by Eqs. (47) and (48). The $1500-\mathrm{keV}$ run was omitted from the averaging, since its low-energy portion is evidently in error. The model fits the rest of the data very well (see Fig. 31).

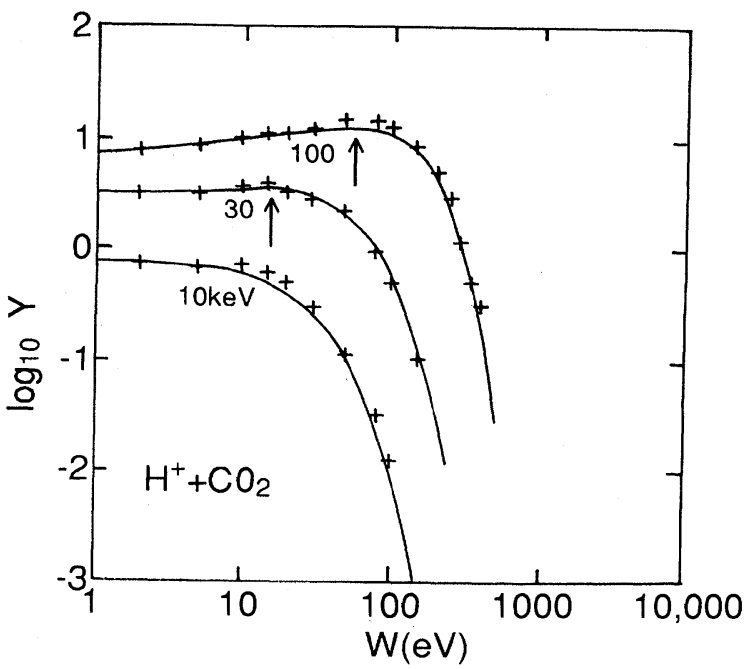

FIG. 30. Energy distribution of secondary electrons from proton collisions on carbon dioxide: + , Cheng et al. (1989b). Rest of legend as in Fig. 23.

\section{J. Methane}

At the time the fitting was done, data sets were available for methane only at four proton energies, one at $T_{0}=50 \mathrm{keV}$ by Gibson and Reid (1987a, 1987b), and runs at 300,400 , and $500 \mathrm{keV}$ by Stolterfoht (1971a). While this range of energies is not large enough to provide reliable parameters, a set that should be approximately correct has been selected with the help of the total cross sections. The results are estimated to have a $25 \%$ uncertainty above $50 \mathrm{keV}$ and $40 \%$ below. Figure 32 shows some of the results.

\section{K. Other targets}

None of the other targets had data over a sufficient energy range to yield parameters for the Rudd model.

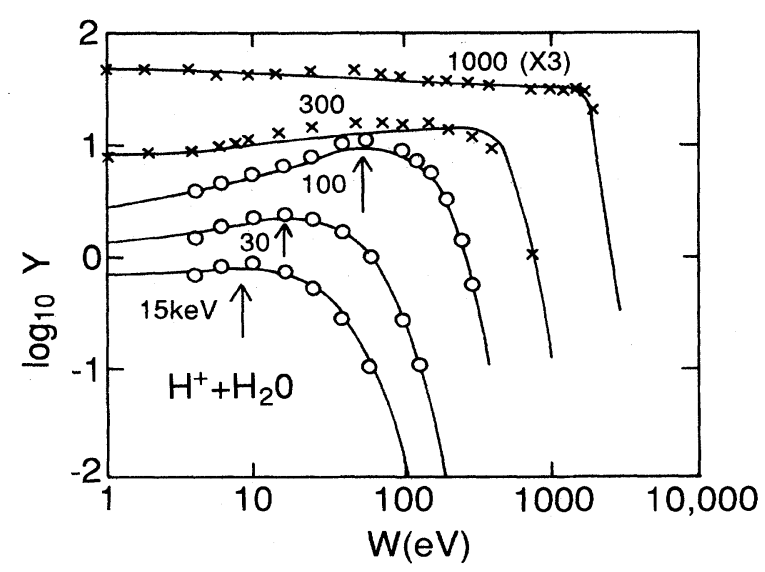

FIG. 31. Energy distribution of secondary electrons from proton collisions on water vapor: $\bigcirc$, Bolorizadeh and Rudd (1986); $\times$, Toburen and Wilson (1977). Rest of legend as in Fig. 23. 


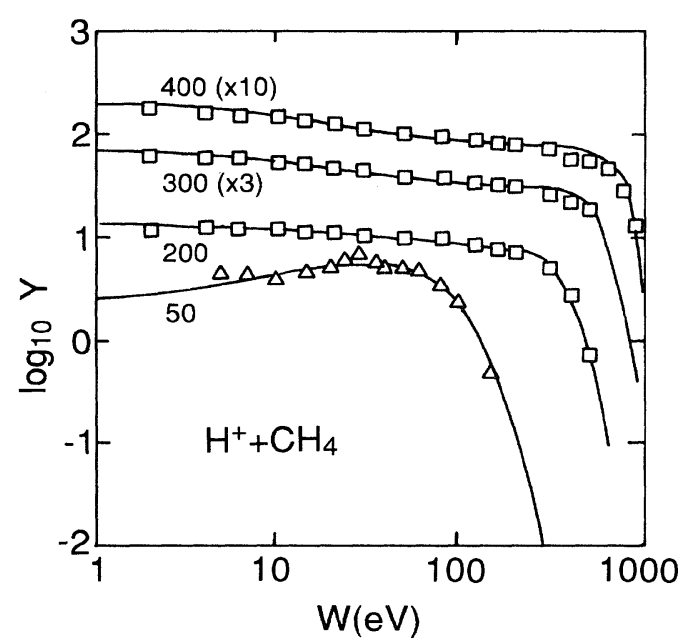

FIG. 32. Energy distribution of secondary electrons from proton collisions on methane: $\triangle$, Gibson and Reid (1987a,b); $\square$, Stolterfoht (1971a).

\section{RECOMMENDATIONS FOR FUTURE WORK}

In this section we briefly review what appear to be the most promising and important avenues for future theoretical and experimental investigations.

Perhaps the most serious drawback in the theory of proton-impact ionization is the lack of convenient theoretical methods to predict cross sections for slow incident protons, i.e., $T_{0} \lesssim 300 \mathrm{keV}$. In addition to the fact that the usual perturbative calculations fail for slow protons, the "two-center" aspects of the collision can now be very important. This necessitates the use of quasimolecular models or theories, which explicitly include the interaction between the ionized electron and the two positive ions, such as the Coulomb distorted-wave method. One approach that is likely to work well in this regime is the classical-trajectory Monte Carlo method described in Sec. IV.B. Hence a systematic study of the doubly- and singly-differential cross sections based on the classicaltrajectory Monte Carlo method for a variety of targets is desirable. Such a study could identify systematics and make it possible to develop more realistic theoretical models that could effectively cover slow incident protons. In general, DDCS measurements made at very low $\left(T_{0}<50 \mathrm{keV}\right)$ energies, where two-center effects are most prominent, have large uncertainties. This is partly due to the fact that the secondary electrons have correspondingly lower energy and are thus difficult to measure accurately, and partly due to the fact that low-energy proton beams are more difficult to characterize and control. It would thus be desirable, from the standpoint of developing theory in this difficult region, to make several careful "benchmark" measurements of SDCS's.

Although first-order perturbation theory such as the Born approximation is valid for fast protons, it is unrealistic to use the PWBA to calculate doubly-differential or singly-differential cross sections for molecules because of the complexity in calculating continuum wave functions for such targets. With the possible exception of $\mathbf{H}_{2}$, it is safer to rely on a combination of semiempirical models and some selective experimental data for the time being. Models based on the Bethe theory (see Sec. VI) will be able to provide reliable predictions for high-energy protons as long as realistic dipole oscillator strengths are used. Such models, however, cannot be built from optical data alone; the models contain parameters that can be determined only if actual proton-impact cross sections are known for some strategic incident energies, e.g., $T_{0}=300,1000,3000$, and $5000 \mathrm{keV}$. When reliable absolute values of the total ionization cross sections are known, experimental DDCS's or SDCS's need not be absolute, since relative cross sections can easily be normalized using Platzman plots. Hence experimental data for DDCS's that emphasize the correct shape rather than absolute magnitude are desirable for targets whose total ionization cross sections and dipole oscillator strengths are well known. Experimental photoionization cross sections, including partial cross sections that identify different ionic states, are also needed to provide differential oscillator strengths for this type of normalization, as well as for modeling collisions with fast incident protons.

Another weak link in the theory concerns multiple ionization. Multiple ionizations that result from a series of single ionization events (such as the ionization of an inner-shell electron followed by Auger electrons created in filling the inner-shell vacancy) can be handled within existing theory. However, no good theory exists for true multiple ionizations, i.e., when two or more electrons from a given subshell are ejected as a result of a single collision. Such multiple ionization is a manifestation of electron-correlation effects, which are inherently nonlinear. Any attempt to describe multiple ionization as a series of single collisions, e.g., the first ejected electron hitting and ionizing another in the same subshell, and so on, will not be sufficient unless such a model includes all orders of perturbation. The development of a true multiple-ionization theory is a worthwhile challenge; such a theory might share the same basis as a nonperturbative theory of multiphoton ionization. Experimentally, measurements of SDCS that are shell specific would be beneficial here. Such data would also be very helpful in providing input parameters for semiempirical models. The level of complexity of such experiments, however, is significantly greater than that of those described in this review, since coincidence detection would be required.

As we have mentioned earlier, experimental difficulties so far have hindered the determination of accurate DDCS's for slow ejected electrons, $W \leq 10 \mathrm{eV}$. The development of any theory that can predict an accurate DDCS for slow electrons ejected from, say, He will provide a valuable tool that not only yields sorely needed data but also serves as a gauge with which experimental distortions can be identified. In combination with such a 
theory, more extensive measurements of low-energy electrons using the time-of-flight technique would dramatically improve the existing data sets.

A judicious application of the theory for Compton profiles may lead to a reliable method to predict the singly-differential cross sections of very fast ejected electrons beyond the classical cutoff. Such electrons contribute little to the total ionization cross sections, but their role becomes more significant in modeling energy deposition by energetic protons.

Experimentally, a number of technical possibilities and challenges exist that hold promise for major improvements in the SDCS data base. As mentioned earlier, benchmark measurements at low primary energy and extensive time-of-flight measurements at low secondary energies are highly desirable. In addition, the technology for measuring subshell-specific DDCS's is currently available, using coincidence measurements, and such measurements should be performed. Two techniques for measuring SDCS's directly-ion energy-loss spectroscopy (Park et al.) and the angle-integrating method of Vroom et al. (1977) - have found only limited use to date. The analyzer of Vroom et al. could be adapted easily for incident-proton measurements. Ion energy-loss measurements, which have been made for a limited range of both primary and secondary energies, for only atomic hydrogen and helium targets, could provide a broad range of direct, reliable SDCS's. While the cross sections measured with ion energy-loss spectroscopy are not strictly equivalent to those measured by secondary-electron analysis, the energy regions where valid comparisons can be made are large, and the two techniques could provide important consistency checks for each other.

If realized, the theoretical and experimental advances just mentioned will significantly improve the applicability of basic ionization physics to problems that need these results. One of the most important of these is radiation damage in biological systems. In this field, more doublydifferential and singly-differential cross sections for a range of hydrocarbon targets would be immediately useful.

\section{ACKNOWLEDGMENTS}

The authors would like to thank Dr. Dave Schultz and Dr. C. O. Reinhold for providing the classical-trajectory Monte Carlo results for helium, Dr. L. H. Toburen for providing numerical tables of his experimental results and for suggested changes in the manuscript, Dr. W. Stevens for providing theoretical energies in Tables I(a) and $\mathbf{I}(\mathrm{b})$, and Dr. J. Berkowitz for references to experimental binding energies. This work was supported in part by NSF Grants PHY-8701905 and PHY-9020529 (M.E.R.) and PHY-9045643 (D.H.M.), and the Office of Fusion Energy of the DOE (Y.-K.K. and T.J.G.).

\section{APPENDIX A: MAXIMUM ENERGY TRANSFERRED TO AN ELECTRON AT REST}

Consider a head-on, linear collision between an incident proton and a free electron initially at rest. Then, the conservation of energy and momentum requires

$$
\begin{aligned}
& M v_{0}^{2} / 2=M v_{p}^{2} / 2+m v_{e}^{2} / 2, \\
& M v_{0}=M v_{p}+m v_{e},
\end{aligned}
$$

where $M$ is the proton mass, $v_{0}$ its incident speed, $v_{p}$ its speed after the collision, $m$ the electron mass, and $v_{e}$ the electron speed after the collision. From Eqs. (A1) and (A2), we get

$$
v_{0}+v_{p}=v_{e} .
$$

However, energy conservation prohibits the proton from moving faster after the collision than its initial speed, i.e., $v_{p} \leq v_{0}$ :

$$
v_{e} \leq 2 v_{0} .
$$

Thus the maximum energy the free electron can gain from the incident proton is

$$
W_{\max }(p)=m v_{e}(\max )^{2} / 2=2 m v_{0}^{2}=4 T,
$$

where

$$
T=m v_{0}^{2} / 2
$$

is the kinetic energy of an electron moving with the same speed as the incident proton.

Note that (a) $W_{\max }(p)$ given by Eq. (A5) is independent of the proton mass and indeed applies to all heavy incident particles [see Eq. (10)]; (b) this limit is far smaller than the actual incident energy, $T_{0}=(M / m) T$; and (c) the maximum kinetic energy of an electron ejected away from the incident-beam direction will be even less than $4 T$ because, in such a case, the momentum transferred to the electron will be smaller.

\section{APPENDIX B: BINARY PEAK ANGLE}

Consider the collision of an incident particle of energy $T_{0}$ and momentum $k_{0}$ with an electron at rest (see Fig. 33). After the collision, the electron recoils at an angle $\theta$ with kinetic energy $W$ and corresponding momentum $k_{e}$, while the proton is scattered at an angle $\phi$ with kinetic energy $T_{p}=T_{0}-W$ and corresponding momentum $k_{p}$.

From momentum conservation, we have

$$
\begin{aligned}
& k_{p} \cos \phi+k_{e} \cos \theta=k_{0}, \\
& k_{p} \sin \phi=k_{e} \sin \theta .
\end{aligned}
$$

By eliminating $\phi$ in Eqs. (B1) and (B2), we get

$$
2 k_{0} k_{e} \cos \theta=k_{0}^{2}+k_{e}^{2}-k_{p}^{2},
$$

while

$$
\begin{aligned}
& k_{0}^{2}=2 M T_{0}, \quad k_{e}^{2}=2 m W, \\
& k_{p}^{2}=2 M T_{p}=2 M\left(T_{0}-W\right) .
\end{aligned}
$$

Substitution of Eq. (B4) into Eq. (B3) results in 


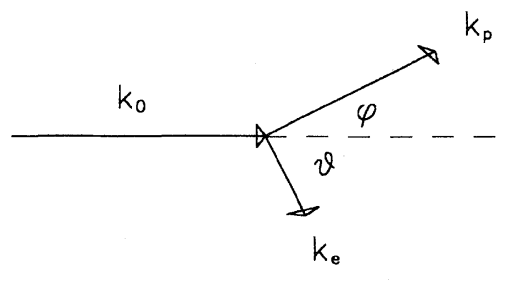

FIG. 33. Momenta in the collision of a proton with an electron at rest. $k_{0}$, incident proton momentum; $k_{p}$, scattered proton momentum; $k_{e}$, ejected electron momentum.

$$
\begin{aligned}
\cos \theta_{b} & =\left(W / T_{0}\right)^{1 / 2} \quad \text { if } M=m, \\
& =\frac{1}{2}\left(M W / m T_{0}\right)^{1 / 2} \text { if } M \gg m,
\end{aligned}
$$

where $\theta_{b}$ is the angle to which the (secondary) electron is ejected. Since the target electron was at rest initially, $\theta_{b}$ is the only direction it can go, i.e., it has a delta-function angular distribution.

From Eq. (B6), we get

$$
W=4 T \cos ^{2} \theta_{b} \text {. }
$$

Hence an electron ejected in the forward direction has the maximum energy $W_{\max }=4 \mathrm{~T}$.

\section{REFERENCES}

Abrines, R., and I. C. Percival, 1966a, Proc. Phys. Soc. London 88, 861 .

Abrines, R., and I. C. Percival, 1966b, Proc. Phys. Soc. London 88, 873 .

Andersen, H. H., and H. L. Bay, 1981, in Sputtering by Particle Bombardment I, Springer Topics in Applied Physics No. 47, edited by R. Behrisch (Springer, Berlin).

Arcuni, P. W., and D. Schneider, 1987, Phys. Rev. A 36, 3059, and references 1 and 23 therein.

Bang, J., and J. M. Hansteen, 1959, K. Dan. Vidensk. Selsk. Mat. Fys. Medd. 31, No. 13.

Belkić, Dž., 1978, J. Phys. B 11, 3529.

Benoit-Cattin, P., D. Blanc, A. Bordenave-Montesquieu, A. Gleizes, M. Rodière, and S. Sobhanian, 1973, J. Phys. E 6, 1.

Berkowitz, J., 1979, Photoabsorption, Photoionization, and Photoelectron Spectroscopy (Academic, New York).

Bernardi, G., P. Fainstein, C. R. Garibotti, and S. Suarez, 1990, J. Phys. B 23, L139.

Bernardi, G., S. Suarez, P. Focke, and W. Meckbach, 1988, Nucl. Instrum. Methods B 33, 321.

Bernardi, G. C., S. Suarez, P. D. Fainstein, C. R. Garibotti, W. Meckbach, and P. Focke, 1989, Phys. Rev. A 40, 6863.

Bethe, H., 1930, Ann. Phys. (Leipzig) 5, 325.

Bethe, H. A., and R. W. Jackiw, 1986, Intermediate Quantum Mechanics, 3rd ed. (Benjamin, New York).

Bevington, Philip R., 1969, Data Reduction and Error Analysis for the Physical Sciences (McGraw-Hill, New York).

Blaauw, H. J., R. W. Wagenaar, D. H. Barends, and F. J. DeHeer, 1980, J. Phys. B 13, 359.

Blauth, E., 1957, Z. Phys. 147, 228.

Bolorizadeh, M. A., and M. E. Rudd, 1986, Phys. Rev. A 33, 888 .
Bonsen, T. F. M., and D. Banks, 1971, J. Phys. B 4, 706.

Bonson, T. F. M. and L. Vriens, 1970, Physica 47, 307.

Bordenave-Montesquieu, Alain, 1973, D.S. thesis, l'Université

Paul Sabattier de Toulouse.

Bordenave-Montesquieu, A., A. Gleizes, and P. Benoit-Cattin, 1982, Phys. Rev. A 25, 245.

Bordenave-Montesquieu, A., A. Gleizes, M. Rodière, and P. Benoit-Cattin, 1973, J. Phys. B 6, 1997.

Bordoni, F., 1971, Nucl. Instrum. Methods 97, 405.

Bottcher, C., 1982, Phys. Rev. Lett. 48, 85.

Brüche, E., 1927, Ann. Phys. (Leipzig) 83, 1065.

Cacak, R. K., and T. Jorgensen, Jr., 1970, Phys. Rev. A 2, 1322.

Cheng, W.-Q., M. E. Rudd, and Y.-Y. Hsu, 1989a, Phys. Rev. A 39, 2359.

Cheng, W.-Q., M. E. Rudd, and Y.-Y. Hsu, 1989b, Phys. Rev. A 40, 3599.

Cheshire, I. M., 1964, Proc. Phys. Soc. London 84, 89.

Clementi, E., and D. L. Raimondi, 1963, J. Chem. Phys. 38, 2686.

Clementi, E., D. L. Raimondi, and W. P. Reinhardt, 1967, J. Chem. Phys. 47, 1300.

Cook, C. J., 1955, Rev. Sci. Instrum. 26, 92.

Criswell, T. L., L. H. Toburen, and M. E. Rudd, 1977, Phys. Rev. A 16, 508.

Crooks, J. B., and M. E. Rudd, 1970, Phys. Rev. Lett. 25, 1599.

Crooks, J. B., and M. E. Rudd, 1971, Phys. Rev. A 3, 1628.

Crothers, D. S. F., and J. F. McCann, 1983, J. Phys. B 16, 3229.

DuBois, R. D., 1985, Phys. Rev. A 32, 3319.

DuBois, R. D., 1986, Phys. Rev. A 34, 2738.

DuBois, R. D., and S. T. Manson, 1987, Phys. Rev. A 35, 2007.

Dunning, T. H., and P. J. Hay, 1977, in Methods of Electronic Structure Theory, edited by H. F. Shaefer III (Plenum, New York), p. 1.

Dyke, J., N. Jonathan, E. Lee, and A. Morris, 1976, J. Chem. Soc. Faraday Trans. II 72, 1385.

Fainstein, P. D., V. H. Ponce, and R. D. Rivarola, 1988a, J. Phys. B 21, 287.

Fainstein, P. D., V. H. Ponce, and R. D. Rivarola, 1988b, J. Phys. B 21, 2989.

Fainstein, P. D., V. H. Ponce, and R. D. Rivarola, 1989a, Phys. Rev. A 40, 2828.

Fainstein, P. D., V. H. Ponce, and R. D. Rivarola, 1989b, J. Phys. B 22, L559.

Fainstein, P. D., V. H. Ponce, and R. D. Rivarola, 1991, J. Phys. B 24, 3091.

Fano, U., and J. W. Cooper, 1968, Rev. Mod. Phys. 40, 441.

Ford, A. L., J. F. Reading, and R. L. Becker, 1981, Phys. Rev. A 23, 510.

Franco, V., 1968, Phys. Rev. Lett. 20, 709.

Fritsch, W., and C. D. Lin, 1983, Phys. Rev. A 27, 3361.

Gabler, H., 1974, Ph.D. thesis, Free University, Berlin.

Garibotti, C. R., and J. E. Miraglia, 1980, Phys. Rev. A 21, 572.

Gay, T. J., M. W. Gealy, and M. E. Rudd, 1990, J. Phys. B 23, L823.

Gerjuoy, E., 1966, Phys. Rev. 148, 54.

Gibson, D. K., and I. D. Reid, 1984, J. Phys. E 17, 1227.

Gibson, D. K., and I. D. Reid, 1985, Australian Atomic Energy Commission Report AAEC/E608.

Gibson, D. K., and I. D. Reid, 1986, J. Phys. B 19, 3265.

Gibson, D. K., and I. D. Reid, 1987a, Australian Atomic Energy Commission Report AAEC/E659.

Gibson, D. K., and I. D. Reid, 1987b, Radiat. Res. 112, 418.

Gibson, S. T., J. P. Greene, and J. Berkowitz, 1985, J. Chem. Phys. 83, 4319. 
Glauber, R. J., 1959, in Lectures in Theoretical Physics, Vol. I, edited by W. E. Brittin and L. G. Dunham (Interscience, New York), p. 315.

Golden, D. E., and H. W. Bandel, 1965, Phys. Rev. 138, 14A.

Golden, D. E., H. W. Bandel, and J. A. Salerno, 1966, Phys. Rev. 146, 40.

Golden, J. E., and J. H. McGuire, 1974, Phys. Rev. Lett. 32, 1218.

Golden, J. E., and J. H. McGuire, 1977, Phys. Rev. A 15, 499.

Gryzinski, M., 1959, Phys. Rev. 115, 374.

Gryzinski, M., 1965a, Phys. Rev. 138, 305A.

Gryzinski, M., 1965b, Phys. Rev. 138, 327A.

Gryzinski, M., 1965c, Phys. Rev. 138, 336A.

Hansteen, J. M., and O. P. Mosebekk, 1972, Phys. Rev. Lett. 29, 1361.

Inokuti, M., 1971, Rev. Mod. Phys. 43, 297.

Inokuti, M., M. A. Dillon, J. H. Miller, and K. Omidvar, 1987, J. Chem. Phys. 87, 6967.

Irby, V. D., T. J. Gay, J. Wm. Edwards, E. B. Hale, M. L. McKenzie, and R. E. Olson, 1988, Phys. Rev. A 37, 3612.

Janev, R., K., and L. P. Presnyakov, 1980, J. Phys. B 13, 4233.

Kim, Y.-K., 1975a, Radiat. Res. 61, 21.

Kim, Y.-K., 1975b, Radiat. Res. 64, 96.

Kim, Y.-K., 1975c, Radiat. Res. 64, 205.

Kim, Y.-K., 1975d, Int. J. Radiat. Phys. Chem. 7, 77.

Kim, Y.-K., 1976, in The Physics of Electronic and Atomic Collisions, edited by J. R. Risley and R. Geballe (University of Washington, Seattle), p. 741.

Kim, Y.-K., 1983, in Physics of Ion-Ion and Electron-Ion Collisions, edited by F. Brouillard and J. W. McGowan (Plenum, New York), p. 101.

Kim, Y.-K., and J. P. Desclaux, 1988, Phys. Rev. A 38, 1805.

Kim, Y.-K., and M. Inokuti, 1971, Phys. Rev. A 3, 665.

Kimura, M., and M. Inokuti, 1988, Phys. Rev. A 38, 3801.

Knudsen, M., 1910, Ann. Phys. (Leipzig) 31, 205.

Kuyatt, C. E., 1968, in Methods of Experimental Physics, Vol. 7, Part A. edited by Benjamin Bederson and Wade L. Fite (Academic, New York), p. 1.

Kuyatt, C. E., and T. Jorgensen, Jr., 1963, Phys. Rev. 130, 1444. Landau, L., and E. M. Lifshitz, 1965, Quantum Mechanics, Non-Relativistic Theory, 2nd ed. (Pergamon, London), p. 519.

Lynch, D. J., L. H. Toburen, and W. E. Wilson, 1976, J. Chem. Phys. 64, 2616.

Macek, J., 1970, Phys. Rev. A 1, 235.

Madison, D. H., and E. Merzbacher, 1975, in Atomic InnerShell Processes, edited by B. Crasemann (Academic, New York), p. 1.

Manson, S. T., and L. H. Toburen, 1977, in Tenth International Conference on the Physics of Electronic and Atomic Collisions, Abstracts (Commissariat à l'Energie Atomique, Paris), p. 990.

Manson, S. T., L. H. Toburen, D. H. Madison, and N. Stolterfoht, 1975, Phys. Rev. A 12, 60.

Mashkova, E. S., and V. A. Molchanov, 1985, Medium-Energy Ion Reflection from Solids (North-Holland, Amsterdam).

McGuire, J. H., 1982, Phys. Rev. A 26, 143.

McGuire, J. H., and L. Weaver, 1977, Phys. Rev. A 16, 41.

Miller, J. H., W. E. Wilson, S. T. Manson, and M. E. Rudd, 1987, J. Chem. Phys. 86, 157.

Miller, W. F., and R. L. Platzman, 1957, Proc. Phys. Soc. London, Ser. A 70, 299.

Moore, J. H., C. C. Davis, and M. A. Coplan, 1983, Building Scientific Apparatus (Addison-Wesley, London).

Mott, N. F., and H. S. W. Massey, 1965, The Theory of Atomic Collisions, (Oxford University, New York/London), Chap. III.
Mukoyama, T., C. D. Lin, and W. Fritsch, 1985, Phys. Rev. A 32, 2490.

Normand, C. E., 1930, Phys. Rev. 35, 1217.

Olson, R. E., 1983, Phys. Rev. A 27, 1871.

Olson, R. E., and T. J. Gay, 1988, Phys. Rev. Lett. 61, 302.

Olson, R. E., T. J. Gay, H. G. Berry, E. B. Hale, and V. D. Irby, 1987, Phys. Rev. Lett. 59, 36.

Olson, R. E., and A. Salop, 1977, Phys. Rev. A 16, 531.

Opal, C. B., E. C. Beaty, and W. K. Peterson, 1972, At. Data 4, 209.

Palmer, D. W., M. W. Thompson, and P. D. Townsend, 1970, Eds., Atomic Collision Phenomena in Solids (NorthHolland/American Elsevier, Amsterdam/New York).

Park, J. T., 1983, Adv. At. Mol. Phys. 19, 67.

Park, J. T., J. E. Aldag, J. M. George, and J. L. Peacher, 1976, Phys. Rev. A 14, 608.

Park, J. T., J. E. Aldag, J. M. George, J. L. Peacher, and J. H. McGuire, 1977, Phys. Rev. A 15, 502.

Park, J. T., J. M. George, J. L. Peacher, and J. E. Aldag, 1978, Phys. Rev. A 18, 48.

Park, J. T., E. Redd, T. J. Kvale, and E. Rille, 1983, Rev. Sci. Instrum. 54, 1247.

Park, J. T., and F. D. Schowengerdt, 1969a, Rev. Sci. Instrum. 40, 753.

Park, J. T., and F. D. Schowengerdt, 1969b, Phys. Rev. 185, 152.

Paul, H., and W. Obermann, 1983, Nucl. Instrum. Methods 214, 15.

Potts, A. W., H. J. Lempka, D. G. Streets, and W. C. Price, 1970, Philos. Trans. R. Soc. London, Ser. A 268, 59.

Reading, J. F., and A. L. Ford, 1979, J. Phys. B 12, 1367.

Reading, J. F., and A. L. Ford, 1987, J. Phys. B 20, 3747.

Reading, J. F., A. L. Ford, and R. L. Becker, 1981, J. Phys. B 14, 1995.

Reading, J. F., A. L. Ford, G. L. Swafford, and A. Fritchard, 1979, Phys. Rev. A 20, 130.

Reinhold, C. O., and C. A. Falcon, 1986, Phys. Rev. A 33, 3859.

Reinhold, C. O., C. A. Falcon, and J. E. Miraglia, 1987, J. Phys. B 20, 3737.

Reinhold, C. O., and R. E. Olson, 1989, Phys. Rev. A 39, 3861.

Rødbro, M., and F. D. Andersen, 1979, J. Phys. B 12, 2883.

Rudd, M. E., 1972, in Low Energy Electron Spectrometry by

Kenneth D. Sevier (Wiley-Interscience, New York), p. 17.

Rudd, M. E., 1977, data listed in Criswell et al., 1977, and

Rudd et al., 1979.

Rudd, M. E., 1979, Phys. Rev. A 20, 787.

Rudd, M. E., 1987, Radiat. Res. 109, 1.

Rudd, M. E., 1988, Phys. Rev. A 38, 6129.

Rudd, M. E., D. Gregoire, and J. B. Crooks, 1971, Phys. Rev. A 3, 1635.

Rudd, M. E., and T. Jorgensen, Jr., 1963, Phys. Rev. 131, 666.

Rudd, M. E., Y.-K. Kim, D. H. Madison, and J. W. Gallagher, 1985, Rev. Mod. Phys. 57, 965.

Rudd, M. E., and D. H. Madison, 1976, Phys. Rev. A 14, 128.

Rudd, M. E., C. A. Sautter, and C. L. Bailey, 1966, 151, 20.

Rudd, M. E., L. H. Toburen, and N. Stolterfoht, 1976, At. Data

Nucl. Data Tables 18, 413.

Rudd, M. E., L. H. Toburen, and N. Stolterfoht, 1979, At. Data

Nucl. Data Tables 23, 405.

Rutherford, E., 1911, Philos. Mag. 21, 669.

Ryufuku, H., 1982, Phys. Rev. A 25, 720.

Samson, J. A. R., and J. L. Gardner, 1973, J. Geophys. Res. 78, 3663.

Samson, J. A. R., T. Masuoka, P. N. Pareek, and G. C. Angel, 
1987, J. Chem. Phys. 86, 6128.

Sataka, M., J. Urakawa, and N. Oda, 1979, J. Phys. B 12, L729.

Schader, J., R. Latz, M. Burkhard, H. J. Frischkorn, D. Hofmann, P. Koschar, K. O. Groeneveld, D. Berenyi, A. Köver, and Gy. Szabo, 1986, Il Nuovo Cimento 7, 219, and reference 10 therein.

Schram, B. L., A. J. H. Boerboom, W. Kleine, and J. Kistemaker, 1965, Physica 32, 749.

Schultz, D. R., 1989, Phys. Rev. A 40, 2330.

Schultz, D. R., and C. O. Reinhold, 1989, private communication.

Shakeshaft, R., 1978, Phys. Rev. A 18, 1930.

Slater, J. C., 1930, Phys. Rev. 36, 57.

Stevens, W. J., H. Basch, and M. Krauss, 1984, J. Chem. Phys. 81, 6026.

Stolterfoht, N., 1971a, Z. Phys. 248, 81.

Stolterfoht, N., 1971b, Z. Phys. 248, 92.

Stolterfoht, N., 1975, data listed in Manson et al., 1975.

Stolterfoht, N., H. Gabler, and U. Leithaüser, 1976, 1979, data listed in Rudd et al., 1979.

Thomas, E. W., 1985, Atomic Data for Controlled Fusion Research Vol. III. "Particle Interaction with Surfaces," Oak Ridge National Laboratory Report No. ORNL-6088/V3.

Thomas, L. H., 1927, Proc. Cambridge Philos. Soc. 23, 713.

Thomson, J. J., 1912, Philos. Mag. 23, 449.

Toburen, L. H., 1971, Phys. Rev. A 3, 216.
Toburen, L. H., 1974, Phys. Rev. A 9, 2505.

Toburen, L. H., 1990, private communication.

Toburen, L. H., S. T. Manson, and Y.-K. Kim, 1978, Phys. Rev. A 17, 148.

Toburen, L. H., and W. E. Wilson, 1972, Phys. Rev. A 5, 247.

Toburen, L. H., and W. E. Wilson, 1975, Rev. Sci. Instrum. 46, 851.

Toburen, L. H., and W. E. Wilson, 1977, J. Chem. Phys. 66, 5202.

Toburen, L. H., W. E. Wilson, and L. E. Porter, 1977, J. Chem. Phys. 687, 4212.

Tokuro, N., and N. Oda, 1985, J. Phys. B 18, 1771.

Vovna, V. I., and F. I. Vilesov, 1974, Opt. Spectrosk. 36, 436.

Vriens, L., 1967, Proc. Phys. Soc. London 90, 935.

Vriens, L., 1969, in Case Studies in Atomic Physics, Vol. 1, edited by E. W. McDaniel and M. R. C. McDowell (NorthHolland, Amsterdam), p. 335.

Vroom, D. A., R. L. Palmer, and J. Wm. McGowan, 1977, J. Chem. Phys. 66, 647.

Williams, E. J., 1927, Nature 119, 489.

Wilson, W. E., 1972, Radiat. Res. 49, 36.

Wilson, W. E., and L. H. Toburen, 1975, Phys. Rev. A 11, 1303.

Wilson, W. E., J. H. Miller, L. H. Toburen, and S. T. Manson, 1984, J. Chem. Phys. 80, 5631.

Winter, T. G., and C. D. Lin, 1984, Phys. Rev. A 29, 3071. 